\title{
A cell-centered implicit-explicit Lagrangian scheme for a unified model of nonlinear continuum mechanics on unstructured meshes
}

\author{
Walter Boscheri*a, Simone Chiocchetti ${ }^{\mathrm{b}}$, Ilya Peshkov ${ }^{\mathrm{b}}$ \\ ${ }^{a}$ Department of Mathematics and Computer Science, University of Ferrara, 44121 Ferrara, Italy \\ ${ }^{b}$ Department of Civil, Environmental and Mechanical Engineering, University of Trento, 38123 Trento, Italy
}

\begin{abstract}
A cell-centered implicit-explicit updated Lagrangian finite volume scheme on unstructured grids is proposed for a unified first-order hyperbolic formulation of continuum fluid and solid mechanics, namely the Godunov-PehskovRomenski (GPR) model. The scheme provably respects the stiff relaxation limits of the continuous model at the fully discrete level, thus it is asymptotic preserving. Furthermore, the GCL is satisfied by a compatible discretization that makes use of a nodal solver to compute vertex-based fluxes that are used both for the motion of the computational mesh as well as for the time evolution of the governing PDEs. Second order of accuracy in space is achieved using a TVD piecewise linear reconstruction, while an implicit-explicit (IMEX) Runge-Kutta time discretization allows the scheme to obtain higher accuracy also in time. Particular care is devoted to the design of a stiff ODE solver, based on approximate analytical solutions of the governing equations, that plays a crucial role when the visco-plastic limit of the model is approached. We demonstrate the accuracy and robustness of the scheme on a wide spectrum of material responses covered by the unified continuum model that includes inviscid hydrodynamics, viscous heat conducting fluids, elastic and elasto-plastic solids in multidimensional settings.
\end{abstract}

Keywords: Cell-centered Lagrangian finite volume schemes, asymptotic preserving IMEX schemes, unified model of continuum mechanics, inviscid and viscous fluids, hyperelasticity, hyperbolic PDE with stiff relaxation, Unstructured meshes

\section{Introduction}

The motion of a continuous medium (either fluid or solid) can be described using two different types of reference frames. The first type exploits frames that are co-moving and co-deforming together with the medium and traditionally called Lagrangian frames. The second type is referred to as Eulerian frames of reference that are neither co-moving nor co-deforming with the continuous medium. Each of the two types has its pros and cons when used for numerical simulations. For example, approaches based on the Lagrangian description allow for accurate tracking of material interfaces and thus are dominated in the computational solid mechanics, or even fluid mechanics algorithms aiming to compute hydrodynamics effects when the vorticity field is not too severe. However, Lagrangian methods are impractical for the simulation of phenomena with vorticity-dominated motion or highly distorted patterns of the medium because the computational mesh, which moves with the material, becomes too distorted and eventually gets tangled. On the other hand, Eulerian schemes are the natural choice for simulating complex flows but also provide a framework for modeling interfaces of arbitrary complexity and topology changes if equipped with some interfacecapturing techniques, e.g. $[108,100,6,66,70,28,117]$. Nevertheless, due to the diffusion intrinsically embedded into any Eulerian scheme, the errors in the interface approximation accumulate over time and Lagrangian schemes are preferable when the accuracy of the interface location is critical, e.g. multi-material computations or plasma flows for inertial confinement fusion applications $[26,10]$.

\footnotetext{
${ }^{*}$ Corresponding author

Email addresses: walter.boscheri@unife.it (Walter Boscheri*), simone.chiocchetti@unitn.it (Simone Chiocchetti), ilya.peshkov@unitn.it (Ilya Peshkov)
} 
The first Lagrangian schemes were proposed in [116] using a formulation of the governing equations in primitive variables, which was also employed later in [9, 29]. However, most of the modern Lagrangian finite volume schemes use the conservation form of the equations based on the physically conserved quantities like mass, momentum and total energy in order to compute shock waves properly, see e.g. [87, 106, 79, 31, 119]. The original approach for Lagrangian algorithms makes use of a staggered grid discretization [118, 77, 78], where the velocity is defined at the cell interfaces and the other variables at the cell centers. Staggered schemes are compatible with the second law of thermodynamics thanks to the addition of an artificial viscosity in the internal energy equation allowing the dissipation of kinetic energy into internal energy through shock waves, thus total energy is not conserved. Although strategies to overcome this drawback have been proposed in [29, 38], the development of cell-centered finite volume (FV) Lagrangian schemes dedicated to solve the hydrodynamics equations has started to gain visibility from the seminal works presented in [39, 40, 83]. As a purely Lagrangian formulation, no mass flux is allowed across cell interfaces, and the cells move and deform by considering the effects of the entire neighborhood. Specifically, these Lagrangian methods are based on the definition of a nodal solver which takes into account multiple one-dimensional Riemann problems occurring across all surrounding cells, and eventually uniquely determines the node velocity. Consequently, compatibility between the motion of the cell, thus its volume, and the cell deformation computed through PDEs involving the discretization of the velocity divergence is ensured. This is the so-called Geometrical Conservation Law (GCL) that is nothing but the discrete Gauss theorem that is satisfied by construction in classical Godunov-type finite volume schemes on fixed grids.

Second order extension in space is typically achieved using a piecewise linear reconstruction of the conserved variables, while second order time stepping can be obtained relying on a classical Runge-Kutta scheme [84, 54], a Generalized Riemann problem methodology [79, 27] following the seminal ideas outlined in [8, 50, 25], or the ADER strategy [22], originally proposed in [112, 110, 111] for fixed meshes. Cell-centered finite volume schemes have been extended to 2D and 3D Lagrangian solid mechanics in [82, 72, 27], while curved meshes are considered in [37, 104].

Higher order of accuracy in space was first achieved in [32, 76, 33, 34], where a third order accurate essentially non-oscillatory (ENO) reconstruction operator is introduced into a Godunov-type Lagrangian finite volume scheme. The mesh velocity is simply computed as the arithmetic average of the corner-extrapolated values in the cells adjacent to a mesh vertex and the numerical fluxes across element interfaces are solved at the aid of approximate Riemann solvers, thus these schemes cannot be regarded as Lagrangian methods sensu stricto, since mass flux can in principle take place across cells. In the finite element framework, high order Lagrangian schemes have been developed in $[88,102]$ and also in $[41,42,43]$, who solved the equations for Lagrangian hydrodynamics using high order curvilinear finite element methods. Displacement-based finite element schemes for simulating engineering large strain transient situations can be found in $[60,49]$. Discontinuous Galerkin (DG) methods on polygonal unstructured meshes have been proposed for hydrodynamics in [51, 52], while the equations for solid mechanics in hypo- and hyperelastic formulations have been solved with Lagrangian DG schemes in [74, 75].

Lagrangian methods also may differ concerning the formulation of the governing equations. More precisely, there do exist total and updated Lagrangian schemes. Total Lagrangian schemes rely on the discretization of the time rate of change of the deformation gradient, its determinant and its co-factor and the computations are performed on a fixed mesh. Contrarily, updated Lagrangian methods physically move the computational mesh and the deformation gradient is the Lagrange-Euler mapping which relates the initial and the current mesh location. The solid dynamics equations written under total Lagrangian formulation have been successfully solved in $[57,1,12,55,13,73,61]$, where a cell-centered finite volume computational framework was employed. Hypoelastic solid mechanics models $[115,11]$ have also been numerically solved with Lagrangian methods, see e.g. [118, 53, 82, 101, 35]. However, these models are not compatible with the second law of thermodynamics, contrarily to the hyperelasticity equations. A parallel discussion about hypo- and hyper-elastic models and their resolution can be found for instance in [90].

From the physical viewpoint, all contributions listed so far deal with either fluid or solid mechanics. In the field of fluid mechanics, most of the existing Lagrangian methods are designed for the solution of hydrodynamics equations, i.e. ideal fluids. On the other hand, elastic or nearly incompressible solids are mainly addressed by moving mesh schemes. An attempt to derive a unified formulation of continuum mechanics in first-order hyperbolic form that includes fluid mechanics as well as solid mechanics has been very recently proposed in [93], referred to as GodunovPeshkov-Romenski (GPR) model. This model makes use of relaxation-type PDEs which govern the deformation of the material. The relaxation parameter in the sources determines the inelasticity time scale of the phenomena under consideration, thus permitting to recover either ideal fluids or ideal elastic solids in the model limits. Furthermore, 
because of the continuous transition from stiff to non-stiff time scale, the GPR model is also capable of capturing the behavior of viscous fluids as well as elasto-plastic solids. The numerical solution of this model has been first proposed on fixed meshes in [47], and consequently extended to plasma flows in [48]. In [21], a high order moving mesh scheme is adopted for the solution of the GPR model, which is based on the direct Arbitrary-Lagrangian-Eulerian (ALE) framework introduced in [15] and references therein.

In this work, we aim at developing a cell-centered updated Lagrangian finite volume scheme for the solution of the GPR model. We consider unstructured meshes, composed of simplex control volumes, both in 2D an 3D. The novel algorithm arises from the previous contribution for hydrodynamics [22] and the recent work on Lagrangian hyperelasticity [23]. Moreover, the numerical schemes presented in the aforementioned references are exactly recovered by the new finite volume method in the limits of the GPR model. Indeed, the asymptotic preserving property of the fully discrete scheme is derived to demonstrate the consistency with already existing and well-established Lagrangian schemes, e.g. the EUCCLHYD scheme [83]. Finally, the compatible discretization of the GCL allows the asymptotic limit of the distortion tensor to be consistently retrieved without resorting to staggered meshes as done in [20]. To the knowledge of the authors, this is the first contribution towards the solution of relaxation-type systems of hyperbolic conservation laws in the updated Lagrangian framework. Second order extension in space and time is devised as well. In order to treat the arbitrarily stiff source terms with which the unified model is endowed, while maintaining the asymptotic properties of the model at the discrete level, we employ a semi-analytical time integration scheme for the stiff source terms [36, 107], which yields a second order discretization of the fully coupled system of PDEs thanks to the implicit-explicit coupling obtained at the aid of the class of IMEX Runge-Kutta time stepping techniques $[2,14,89,24]$.

The rest of this article is structured as follows. In Section 2, we present the governing equations of the GPR model. Section 3 is devoted to detail the Lagrangian finite volume scheme, the ODE solver for stiff relaxation sources and the extension of the algorithm to second order of accuracy. The asymptotic analysis of the fully discrete numerical method is developed in Section 4. In Section 5, we show numerical convergence studies and a wide range of test cases on 2D and 3D unstructured moving meshes for different types of materials and different equations of state. Finally, in Section 6 we give some concluding remarks and an outlook to future research and developments.

\section{Governing equations: the GPR model for continuum mechanics}

The unified first-order hyperbolic model of continuum mechanics discussed in this section can describe fluid flows and deformations of solids in a single system of first-order hyperbolic equations. The model originates from the works by Godunov and Romenski in 1970s [58, 56, 96, 59] on the modeling of large elasto-plastic deformations of solids. Similar to the work by Wilkins [118], the important feature of the model is that the description is made in an Eulerian frame of reference in contrast to the conventional Lagrangian description of solid mechanics. Later, in the work by Peshkov and Romenski [93], this allows for further generalization of the Godunov-Romenski model (which would be impossible under the Lagrangian description) towards incorporating the description of viscous fluids as a particular case of "extremely" inelastic deformations of solids, see also [47, 46, 28, 20]. Therefore, in this paper, we shall refer to this model as the Godunov-Peshkov-Romenski (GPR) model.

In contrast to the conventional Navier-Stokes-based fluid mechanics, in the GPR model, fluids are characterized not with a strain-rate measure like in the Newton law of viscosity, but with a deformation measure and a special procedure of relaxation of tangential stresses similar to the Maxwell model of viscoelasticity. Nevertheless, it can be shown via a formal asymptotic analysis that the Navier-Stokes Cauchy stress tensor is recovered in the relaxation limit of the GPR model $[47,20]$. Thus, according to the GPR model, each material element of an isotropic continuum is defined by an infinitesimal frame $\boldsymbol{A}_{e}$ (local basis triad) that characterizes deformation and orientation of the material particles. The frame field $\boldsymbol{A}_{e}$ is also called the effective elastic distortion [59], or simply the distortion field. The relaxed (stress-free) state is defined for each material element individually as a state when the frame field $\boldsymbol{A}_{e}$ is orthonormal. In general, such a state may not be reachable globally for all the material elements simultaneously, hence resulting in residual stresses in the material ${ }^{1}$, see Section 5.8 and 5.9.

\footnotetext{
${ }^{1}$ In conventional fluids with zero yield strength, the residual stresses cannot be generated because the relaxation process acts until all stresses vanish.
} 
The GPR model can be also seen as a finite-strain elasto-plasticity model of hyperelastic type [90]. Thus, according to the traditional finite-strain elasto-plasticity framework, the total deformation gradient $\boldsymbol{F}_{t}$ can be decomposed as $\boldsymbol{F}_{t}=\boldsymbol{F}_{e} \boldsymbol{F}_{p}$ into the elastic $\boldsymbol{F}_{e}$ and plastic $\boldsymbol{F}_{p}$ part. The natural Lagrangian stress, i.e. the first Piola-Kirchhoff stress, is then computed as $\boldsymbol{\Pi}=\frac{\partial E}{\partial \boldsymbol{F}_{t}}$, where $E$ is the total energy potential, which, in fact, must be a function of only the elastic part $\boldsymbol{F}_{e}$ of the deformation gradient, that is $E=E\left(\boldsymbol{F}_{e}\right)$. Hence, $\boldsymbol{\Pi}=\frac{\partial E}{\partial \boldsymbol{F}_{t}}=\frac{\partial E}{\partial \boldsymbol{F}_{e}} \boldsymbol{F}_{p}^{-\top}$, and therefore, to compute the stress tensor in the Lagrangian frame, one needs to know any two of the three quantities $\boldsymbol{F}_{t}, \boldsymbol{F}_{e}$, or $\boldsymbol{F}_{p}$. On the other hand, in the Eulerian frame, the natural Eulerian stress tensor is the Cauchy stress $\boldsymbol{T}=\rho \boldsymbol{F}_{t} \frac{\partial E^{\top}}{\partial \boldsymbol{F}_{t}}$, that can be written as

$$
\boldsymbol{T}=\rho \boldsymbol{F}_{t}{\frac{\partial E^{\top}}{\partial \boldsymbol{F}_{t}}}^{\top}=\rho \boldsymbol{F}_{t} \boldsymbol{F}_{p}^{-1}{\frac{\partial E^{\top}}{\partial \boldsymbol{F}_{e}}}^{\top}=\rho \boldsymbol{F}_{e} \frac{\partial E^{\top}}{\partial \boldsymbol{F}_{e}}
$$

Consequently, to compute the stress tensor in the Eulerian frame, one needs to know only the elastic part $\boldsymbol{F}_{e}$ of the total gradient $\boldsymbol{F}_{t}$. In other words, we do not need to compute the entire history of inelastic deformations stored in $\boldsymbol{F}_{p}$. This opens the great possibility to describe arbitrarily large inelastic deformations typical of fluid-type motions. The distortion field $\boldsymbol{A}_{e}$ can also be seen as the inverse of $\boldsymbol{F}_{e}$, thus $\boldsymbol{A}_{e}=\boldsymbol{F}_{e}^{-1}$.

Apart of being a deformation measure, the frame field $\boldsymbol{A}_{e}$ also encodes the orientational degrees of freedom of the material elements, e.g. see the computational results in $[47,20,91]$. The orientational degrees of freedom might be relevant for turbulence modeling [94] or anisotropic plasticity models [98]. However, in this paper, we ignore these degrees of freedom and instead of the frame field $\boldsymbol{A}_{e}$ we consider the metric tensor $\boldsymbol{G}_{e}=\boldsymbol{A}_{e}^{\top} \boldsymbol{A}_{e}$. However, in the context of structure preserving numerical methods, the formulation in terms of $\boldsymbol{A}_{e}$ is more preferable because the deformation compatibility condition $\nabla \times \boldsymbol{A}_{e}=0$ is linear while, in terms of $\boldsymbol{G}_{e}$, the compatibility condition (vanishing Riemann curvature tensor) is nonlinear, e.g. see [59]. In addition, we also take into account heat conduction effects via a first-order hyperbolic formulation proposed by Romenski in [85, 97], which characterizes the heat propagation via a relaxation equation for the thermal impulse $\mathbf{J}$, e.g. see [47]. In later papers [20, 95, 92], we however, use slightly different hyperbolic heat conduction model that coincides with the one used in this paper in the Fourier limit (local thermodynamic equilibrium).

For the updated Lagrangian scheme discussed in Section 3, we write the Eulerian equations [47] in the co-moving frame of reference, that is the time derivative is the Lagrangian derivative (or material derivative), while the spatial derivatives are the Eulerian ones. The space is defined in $\mathbb{R}^{d}$ with $d=\{2,3\}$ representing the number of space dimensions. The time coordinate is given by $t$, while $\mathbf{x}=(x, y, z)$ denotes the spatial position vector. The system of governing equations for the unknowns $\mathbf{Q}:=\left\{\omega, \mathbf{v}, E, \mathbf{J}, \mathbf{G}_{e}\right\}$ therefore reads

$$
\begin{aligned}
& \rho \frac{\mathrm{d} \omega}{\mathrm{d} t}-\nabla \cdot \mathbf{v}=0, \\
& \rho \frac{\mathrm{d} \mathbf{v}}{\mathrm{d} t}-\nabla \cdot \mathbf{T}=\mathbf{0}, \\
& \rho \frac{\mathrm{d} E}{\mathrm{~d} t}-\nabla \cdot(\boldsymbol{T} \mathbf{v})+\nabla \cdot \mathbf{q}=0, \\
& \rho \frac{\mathrm{d} \mathbf{J}}{\mathrm{d} t}+\nabla T=-\frac{\rho \mathbf{H}}{\Psi}, \\
& \frac{\mathrm{d} \mathbf{G}_{e}}{\mathrm{~d} t}+\mathbf{G}_{e} \nabla \mathbf{v}+\nabla \mathbf{v}^{\top} \mathbf{G}_{e}=\frac{2}{\rho \Theta} \boldsymbol{\sigma},
\end{aligned}
$$

where $\rho$ is the mass density, $\omega=\rho^{-1}$ is the specific volume, $\mathbf{v}=(u, v, w)$ is the velocity, $\boldsymbol{T}$ is the Cauchy stress tensor, $E\left(\rho, p, \mathbf{v}, \mathbf{G}_{e}\right)$ is the total energy

$$
\begin{gathered}
E=E_{h}(\rho, p)+E_{e}\left(\mathbf{G}_{e}\right)+E_{t h}(\mathbf{J})+E_{k}(\mathbf{v}), \\
E_{h}=\varepsilon(\rho, p), \quad E_{e}=\frac{c_{\mathrm{sh}}^{2}}{4}\left\|\stackrel{\circ}{G}_{e}\right\|^{2}, \quad E_{t h}=\frac{1}{2} \alpha^{2}\|\mathbf{J}\|^{2}, \quad E_{k}=\frac{1}{2}\|\mathbf{v}\|^{2},
\end{gathered}
$$


with $E_{h}=\varepsilon(\rho, p)$ being the pure hydrodynamics part, $p$ being the hydrodynamics pressure defined only from the hydrodynamics equation of state $\varepsilon(\rho, p), E_{e}$ being the elastic energy stored in the deformed material elements, with $c_{\mathrm{sh}}$ being the shear sound speed which characterizes the rigidity of the material elements, and $\mathbf{G}_{e}=\mathbf{G}_{e}-\frac{1}{3} \operatorname{tr}\left(\mathbf{G}_{e}\right) \boldsymbol{I}$ being the deviatoric part of the metric tensor $\boldsymbol{G}_{e}$, and $\boldsymbol{I}$ is the identity tensor. The third term $E_{t h}$ represents the energy carried by the thermal impulse $\mathbf{J}$ with $\alpha$ characterizing the velocity of propagation of thermal perturbations (defined later in Section 2.3). The last term $E_{k}$ denotes the standard kinetic energy of the continuous medium. The source terms in (2d) and (2e) are the algebraic relaxation sources defined in Section 2.2 and 2.3. The temperature $T$ in (2d) is not a state variable and must be defined from the equations of state $\varepsilon(\rho, p)$, $\mathbf{q}$ is the heat flux defined later in Section 2.3. The Cauchy stress $\boldsymbol{T}$ is split into two parts:

$$
\boldsymbol{T}=-p \boldsymbol{I}+\boldsymbol{\sigma}, \quad \boldsymbol{\sigma}=-2 \rho \mathbf{G}_{e} \frac{\partial E}{\partial \mathbf{G}_{e}}=-\rho c_{\mathrm{sh}}^{2} \mathbf{G}_{e} \stackrel{\mathfrak{G}}{e}
$$

where the pressure $p$ is defined from the hydrodynamics energy $E_{h}(\rho, p)$ and the tangential (or viscous) stress $\sigma$ results from the definition of the elastic energy $E_{e}\left(\mathbf{G}_{e}\right)$. In all computational examples for viscous fluids and elasto-plastic solids in Section 5, we use $E_{e}$ in the form (3b), simply because the asymptotic analysis for the Navier-Stokes limit in [47] was performed for this particular choice and we aim at demonstrating the asymptotic property for the fully discrete scheme. Nevertheless, other elastic energies could be used. We remark that the spherical part of $\sigma$ for our choice of the elastic energy is not zero but scales as $\sim\left\|\stackrel{G}{e}_{e}\right\|^{2}$, i.e. quadratically in $\dot{G}_{e}$. For example, for fluid flows it is negligibly small, while for hyperelastic solids it might be not small and the total pressure should be defined as $P=-\frac{1}{3} \operatorname{tr}(\boldsymbol{T})=p-\frac{1}{3} \operatorname{tr}(\boldsymbol{\sigma})$.

\subsection{Equation of state for $E_{h}$}

In the numerical examples in Section 5, we use three equations of state (EOS) for the hydrodynamics energy $E_{h}$. For gases, we use the ideal gas EOS:

$$
\varepsilon(\rho, p)=\frac{p}{\rho(\gamma-1)}, \quad T=\frac{\varepsilon}{c_{v}}, \quad c_{0}^{2}=\frac{\gamma p}{\rho},
$$

where $\gamma=c_{p} / c_{v}$ is the ratio of specific heats, $c_{p}$ is the specific heat at constant pressure, $c_{v}$ is the specific heat at constant volume, and $c_{0}$ is the adiabatic sound speed.

For liquids and solids, one can use the Mie-Grüneisen equations of state:

$$
\varepsilon(\rho, p)=\frac{p-\rho_{0} c_{0}^{2} f(J)}{\rho_{0} \Gamma_{0}}, \quad f(J)=\frac{(J-1)\left(J-\frac{1}{2} \Gamma_{0}(J-1)\right)}{(J-s(J-1))^{2}}, \quad J=\frac{\rho}{\rho_{0}},
$$

where $c_{0}=$ const is the adiabatic sound speed, $\Gamma_{0}=$ const, $s=$ const are material constants, and $\rho_{0}$ is the reference mass density.

The third type of hydrodynamics EOS used in Section 5.11 and 5.12 is the volumetric part of the Neo-Hookean hyperelastic EOS that reads

$$
\varepsilon(\rho, p)=\frac{G}{4 \rho_{0}}\left((J-1)^{2}+(\log (J))^{2}\right), \quad p=-\frac{G}{2}\left(J-1+\frac{\log (J)}{J}\right), \quad J=\frac{\rho}{\rho_{0}},
$$

where $G=\rho_{0} c_{\mathrm{sh}}^{2}$ is the shear modulus, which also corresponds to the second Lamé coefficient. Material mechanical properties are often described in terms of Young modulus $Y$ and Poisson ratio $v$. These parameters are linked as follows:

$$
G=\frac{Y}{2(1+v)}
$$

In this case the adiabatic sound speed can be computed as the square root of the bulk modulus $K$, hence

$$
c_{0}^{2}=K=\frac{Y v}{(1+v)(1-2 v)}+\frac{2 G}{3} .
$$




\subsection{Closure for inelastic deformations and fluid flows}

The source terms in (2d) and (2e) describe the dissipative dynamics of the system and must be consistent with the second law of thermodynamics (entropy must be non-decreasing). It can be shown that the specification of the source terms used in this paper guarantees that the entropy is indeed not decreasing, see [47, 92]. Without providing further details (which can be found in [47]) we specify them as follows.

The source term in (2d) handles inelastic deformations of the continuum with the relaxation function $\Theta$ given by

$$
\Theta=\tau_{1} \frac{c_{\mathrm{sh}}^{2}}{3}\left|\boldsymbol{G}_{e}\right|^{-5 / 6},
$$

where $\left|\boldsymbol{G}_{e}\right|=\operatorname{det}\left(\boldsymbol{G}_{e}\right)$ and $\tau_{1}$ is the strain relaxation time which, in general, is a function of state variables $\tau_{1}=$ $\tau_{1}\left(\rho, T, \boldsymbol{G}_{e}\right)$. In particular, $\tau_{1}=$ const for Newtonian fluids, while $\tau_{1} \rightarrow \infty$ for pure elastic solids. The Navier-Stokes stress tensor is recovered in the relaxation limit of system (2), that is $\epsilon=\tau_{1} / t_{\text {macro }} \ll 1$ at first order in $\epsilon$, with $t_{\text {macro }}$ being the macroscopic characteristic time of the physical process. The effective shear viscosity is then given by [47]

$$
\mu=\frac{1}{6} \rho_{0} \tau_{1} c_{\mathrm{sh}}^{2} .
$$

For elasto-plastic solids $\tau_{1}$ can be taken as [5, 90, 21]

$$
\tau_{1}=\tau_{10}\left(\frac{\sigma_{Y}}{\sigma}\right)^{n}, \quad \sigma=\sqrt{\frac{3}{2} \operatorname{tr}\left(\dot{\boldsymbol{\sigma}}^{2}\right)}, \quad \stackrel{\boldsymbol{\sigma}}{=}=\boldsymbol{\sigma}-\frac{1}{3} \operatorname{tr}(\boldsymbol{\sigma}) \boldsymbol{l},
$$

where $\sigma_{Y}$ is the material yield strength under quasi-static loading conditions and $\tau_{10}=$ const is a material-specific constant. The parameter $n$ controls the rate-dependence of the plasticity mode, e.g. the greater $n$ the more the material is rate-independent. For other examples of $\tau_{1}$ suitable for the modeling of non-Newtonian fluids see [91, 65].

\subsection{Heat conduction}

The closure of the heat conducting part of system (2) is also conditioned by the consistency with the second law of thermodynamics $[47,92]$ as well as with the Fourier law of heat conduction. Thus, the relaxation function in the right hand-side of $(2 \mathrm{~d})$ is given by

$$
\Psi=\alpha^{2} \tau_{20} \tau_{2}, \quad \tau_{20}=\frac{\rho}{\rho_{0}} \frac{T_{0}}{T} .
$$

The consistency with the first law of thermodynamics (total energy conservation) requires that the heat flux $\mathbf{q}$ is defined as (see [47])

$$
\mathbf{q}=\alpha^{2} T \mathbf{J} .
$$

At the mechanical and thermodynamical equilibrium, the characteristic velocity of the thermal perturbation propagation is

$$
c_{\mathrm{h}}^{2}=\frac{\alpha^{2}}{\rho_{0}^{2}} \frac{T}{c_{v}} .
$$

The Fourier law of heat conduction $\mathbf{q}=-\kappa \nabla T$ is recovered in the relaxation limit (local equilibrium) with $\tau_{2} \ll t_{\text {macro }}$, at first order in $\tau_{2} / t_{\text {macro }}$. The effective heat conductivity is given by [47]

$$
\kappa=\tau_{2} \alpha^{2} \frac{T_{0}}{\rho_{0}} .
$$

\section{Lagrangian finite volume scheme on unstructured meshes}

\subsection{Discretization of the space-time computational domain}

We start by providing the details about the discretization of the time interval and the spatial computational domain. 
Time computational domain. The time coordinate is defined in the time interval $t \in\left[0, t_{f}\right]$, where $t_{f} \in \mathbb{R}_{0}^{+}$represents the final time. A sequence of discrete points $t^{n}$ approximates the temporal computational domain such that $t \in\left[t^{n} ; t^{n+1}\right]$,

$$
t=t^{n}+\alpha \Delta t^{n}, \quad \alpha \in[0,1],
$$

with $t^{n}$ and $\Delta t^{n}$ denoting the current time and time step, respectively. As done in [79, 27], the time step is limited by a classical CFL stability condition combined with a criterion on the growth rate of the time step, hence

$$
\Delta t^{n}=\min \left(\mathrm{CFL} \cdot \min _{T_{i}} \frac{h_{i}}{a_{i}}, C_{m} \Delta t^{n-1}\right) .
$$

The CFL number is chosen to be CFL $\leq 1 / d$ as usual on unstructured meshes, with $d$ being the number of space dimensions, while $h_{i}=\left|T_{i}\right|^{1 / d}$ is the characteristic mesh size with $\left|T_{i}\right|$ being the volume of the spatial element $T_{i}$. Finally, $a_{i}$ is the maximum eigenvalue estimate (equilibrium estimate) of the governing PDE given by

$$
a_{i}=\left.\sqrt{c_{0}^{2}+\frac{4}{3} c_{\mathrm{sh}}^{2}+c_{\mathrm{h}}^{2}}\right|_{i} .
$$

The criterion for controlling the growth rate of the time step is set with $C_{m}=1.1$, so that the increase of the time step size is mild without sharp discontinuities.

Space computational domain. In the updated Lagrangian framework, the time-dependent computational domain $\Omega(t)$ is discretized at the current time $t^{n}$ by a set of non-overlapping unstructured control volumes $T_{i}^{n}$ with boundary $\partial T_{i}^{n}$, that are given by triangles $(d=2)$ or tetrahedra $(d=3) . N_{E}$ denotes the total number of elements contained in the domain and the union of all elements is called the current tessellation $\mathcal{D}_{\Omega}^{n}$ of the domain

$$
\mathcal{D}_{\Omega}^{n}=\bigcup_{i=1}^{N_{E}} T_{i}^{n} .
$$

To ease notation, we omit the superscript $n$ in the following description, meaning that the spatial domain is discretized as follows at each time level $t^{n}$. The index $i$ refers to the element $T_{i}, f$ addresses a face (which is of dimension $d-1$ ) and $r$ denotes a node. The double index $r i$ is used to express a quantity defined at node $r$ from element $T_{i}$; likewise, $f i$ is used to express a quantity defined at the barycenter of face $f$ from element $T_{i}$. The Neumann neighbor of element $T_{i}$ which shares face $f$ is denoted with $T_{j}$, and the outward pointing unit normal vector is $\mathbf{n}_{f i}$. Finally, the cell volume is $\left|T_{i}\right|$ and the length (2D) or surface (3D) of a face $f$ is addressed with $s_{f}$.

At the aid of Figure 1, the following notation is adopted for the definition of different topological sets:

- $\mathcal{R}_{i}$ is the set of nodes $r$ of element $T_{i}$;

- $\mathcal{R}_{f}$ is the set of nodes $r$ of face $f$;

- $\mathcal{F}_{i}$ is the set of faces $f$ of element $T_{i}$;

- $\mathcal{F}_{r}$ is the set of faces $f$ sharing node $r$;

- $\mathcal{F}_{r i}$ is the set of faces $f$ sharing node $r$ and belonging to element $T_{i}$;

- $\mathcal{T}_{r}$ is the set of elements $T$ sharing node $r$, i.e. the Voronoi neighborhood of node $r$.

Each cell can be divided into subcells $T_{r i}$, that are obtained by connecting the cell centroid with the barycenter of the faces belonging to $\mathcal{F}_{r i}$. The cell centroid $\mathbf{x}_{i}$ is defined as

$$
\mathbf{x}_{i}=\frac{1}{\left|T_{i}\right|} \int_{T_{i}} \mathbf{x} \mathrm{d} \mathbf{x}
$$


while the barycenter of a face $\mathbf{x}_{f}$ is given by

$$
\mathbf{x}_{f}=\frac{1}{N_{r f}} \sum_{r \in R_{f}} \mathbf{x}_{r}
$$

with $N_{r f}$ representing the total number of nodes which share face $f$, i.e. all nodes belonging to $\mathcal{R}_{f}$. Let us now introduce the corner vector $\mathbf{c}_{r i}[40,83]$, which is a geometric object defined at node $r$ within element $T_{i}$ :

$$
\mathbf{c}_{r i}=\frac{1}{d} \sum_{f \in \mathcal{F}_{r i}} s_{f} \mathbf{n}_{f i} .
$$

These corner vectors are a linear combination of the oriented surfaces $s_{f} \mathbf{n}_{f i}$, which assume a linear velocity field over the face so that they remain planar. Therefore, the corner vectors $\mathbf{c}_{r i}$ provide a consistent discretization of the cell boundary $\partial T_{i}$ so that

$$
\sum_{r \in \mathcal{R}_{i}} \mathbf{c}_{r i}=0
$$

which is a second order discretization of Gauss theorem over the control volume $T_{i}$.

Each control volume defined in the physical space $\mathbf{x}$ can be mapped onto a reference element $T_{e}$ in the reference coordinate system $\xi=(\xi, \eta, \zeta)$, see Figure 2. The reference element is the unit triangle in $2 \mathrm{D}$ with vertices $\boldsymbol{\xi}_{1 e}=$ $\left(\xi_{1 e}, \eta_{1 e}\right)=(0,0), \xi_{2 e}=\left(\xi_{2 e}, \eta_{2 e}\right)=(1,0)$ and $\xi_{3 e}=\left(\xi_{3 e}, \eta_{3 e}\right)=(0,1)$, or the unit tetrahedron in 3D with vertices $\boldsymbol{\xi}_{1 e}=\left(\xi_{1 e}, \eta_{1 e}, \zeta_{1 e}\right)=(0,0,0), \boldsymbol{\xi}_{2 e}=\left(\xi_{2 e}, \eta_{2 e}, \zeta_{2 e}\right)=(1,0,0), \xi_{3 e}=\left(\xi_{3 e}, \eta_{3 e}, \zeta_{3 e}\right)=(0,1,0)$ and $\xi_{4 e}=\left(\xi_{4 e}, \eta_{4 e}, \zeta_{4 e}\right)=$ $(0,0,1)$. The spatial mapping in $3 \mathrm{D}$ reads

$$
\mathbf{x}=\mathbf{x}_{1 i}+\left(\mathbf{x}_{2 i}-\mathbf{x}_{1 i}\right) \xi+\left(\mathbf{x}_{3 i}-\mathbf{x}_{1 i}\right) \eta+\left(\mathbf{x}_{4 i}-\mathbf{x}_{1 i}\right) \zeta
$$

where $\mathbf{x}_{k i}=\left(x_{k i}, y_{k i}, z_{k i}\right)$ represents the vector of physical spatial coordinates of the $k$-th vertex of element $T_{i}$ for $k=\{1,2,3,4\}$. Notice that the mapping (25) corresponds to the linear isoparametric approximation of the LagrangeEuler transformation between the deformed (physical) and undeformed (reference) configuration.

\subsection{Integral form of the governing equations}

Let $\Omega(t) \subset \mathbb{R}^{d}$ denote the time-dependent control volume in $d=\{2,3\}$ space dimensions, and $\partial \Omega(t) \subset \mathbb{R}^{d-1}$ its surface with $\mathbf{n}$ being the outward pointing unit normal vector. The time coordinate is $t \in \mathbb{R}_{0}^{+}$and the physical space is
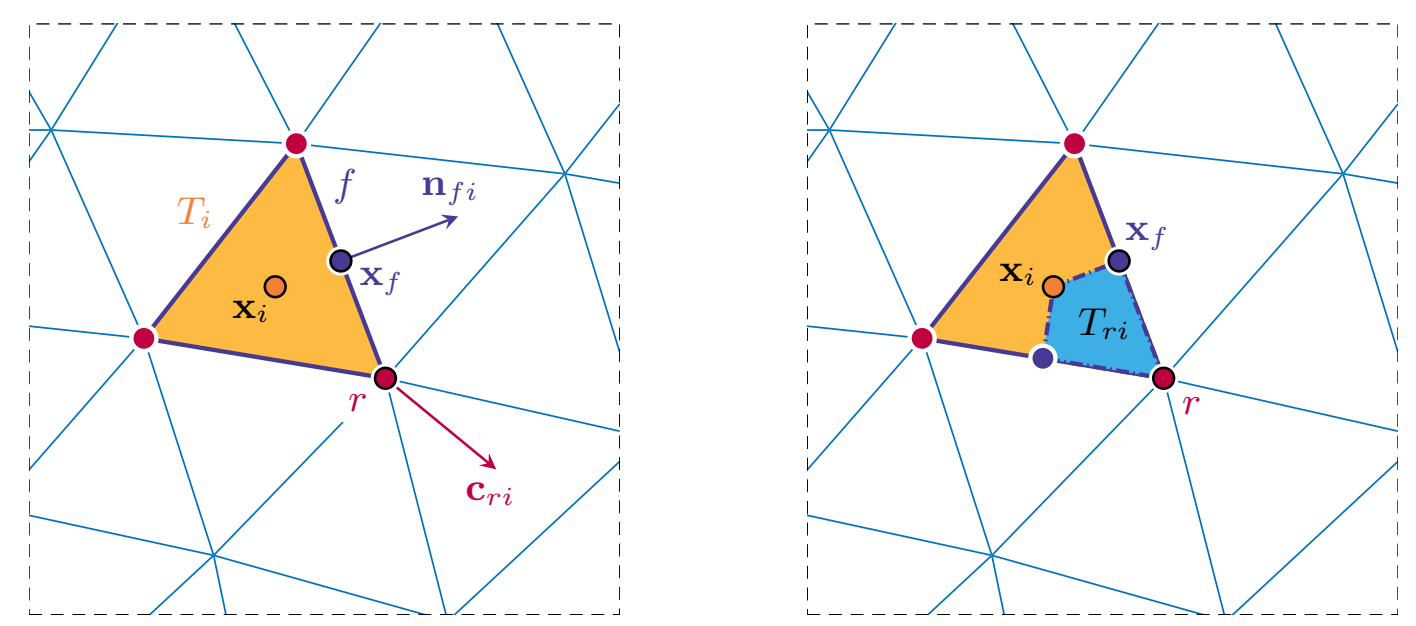

Figure 1: Notation for the mesh. Left: cell barycenter $\mathbf{x}_{i}$ of element $T_{i}$, face barycenter $\mathbf{x}_{f}$ and normal vector $\mathbf{n}_{f i}$ relative to face $f$, corner vector $\mathbf{c}_{r i}$ relative to node $r$. Right: subcell $T_{r i}$ associated with corner $r$ of element $T_{i}$. 
defined by the position vector $\mathbf{x}=(x, y, z)$. To derive a conservative finite volume discretization, the governing equations (28) must be integrated over the control volume $\Omega(t)$. Mass conservation directly follows from the Lagrangian description of continuum mechanics and holds true by construction, that is

$$
\frac{\mathrm{d}}{\mathrm{d} t} \int_{\Omega(t)} \rho \mathrm{d} \mathbf{x}=0 .
$$

After addressing a generic physical variable with $\phi=\phi(\mathbf{x}, t)$, let us introduce the transport relation

$$
\frac{\mathrm{d}}{\mathrm{d} t} \int_{\Omega(t)} \rho \phi \mathrm{d} \mathbf{x}=\int_{\Omega(t)} \rho \frac{\mathrm{d} \phi}{\mathrm{d} t} \mathrm{~d} \mathbf{x},
$$

which is derived from the Reynolds transport theorem [86] using the mass conservation property (26). The control volume formulation of the PDE system (28) can then be expressed as follows:

$$
\begin{aligned}
& \frac{\mathrm{d}}{\mathrm{d} t} \int_{\Omega(t)} \mathrm{d} \mathbf{x}-\int_{\partial \Omega(t)} \mathbf{v} \cdot \mathbf{n} \mathrm{d} s=0, \\
& \frac{\mathrm{d}}{\mathrm{d} t} \int_{\Omega(t)} \rho \mathbf{v} \mathrm{d} \mathbf{x}-\int_{\partial \Omega(t)} \mathbf{T} \mathbf{n} s=\mathbf{0}, \\
& \frac{\mathrm{d}}{\mathrm{d} t} \int_{\Omega(t)} \rho E \mathrm{~d} \mathbf{x}-\int_{\partial \Omega(t)}(\mathbf{T} \mathbf{v}+\mathbf{q}) \cdot \mathbf{n} \mathrm{d} s=0, \\
& \frac{\mathrm{d}}{\mathrm{d} t} \int_{\Omega(t)} \rho \mathbf{J} \mathrm{d} \mathbf{x}+\int_{\partial \Omega(t)} T \mathbf{I n} \mathrm{d} s=-\int_{\Omega(t)} \frac{\rho \mathbf{H}}{\Psi} \mathrm{d} \mathbf{x}, \\
& \int_{\Omega(t)} \mathrm{d} \frac{\mathbf{G}{ }_{e}}{\mathrm{~d} t} \mathrm{~d} \mathbf{x}+\int_{\Omega(t)} \rho\left(\mathbf{G}_{e} \mathbb{L}(\mathbf{v})+\mathbb{L}(\mathbf{v})^{\top} \mathbf{G}_{e}\right) \mathrm{d} \mathbf{x}=\int_{\Omega(t)} \rho \frac{2}{\rho \Theta} \boldsymbol{\sigma} \mathrm{d} \mathbf{x} .
\end{aligned}
$$
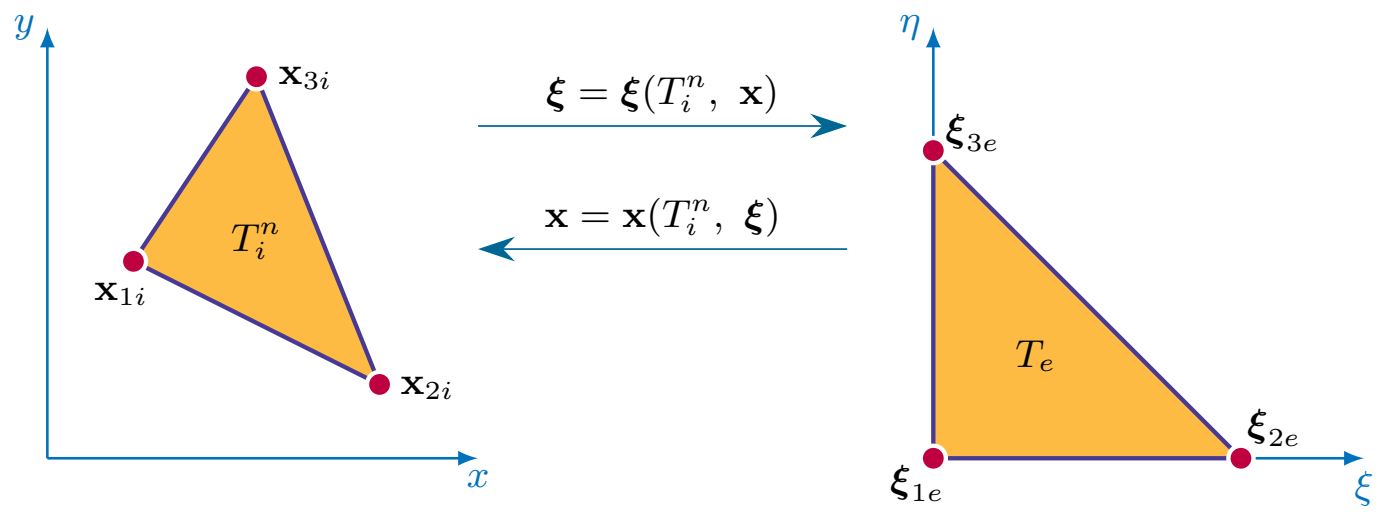

Figure 2: Mapping from the physical element $T_{i}^{n}$ in the global coordinate system $\mathbf{x}=(x, y)$ to the reference element $T_{e}$ in the reference coordinate system $\xi=(\xi, \eta)$. 
Equation (28a) is also referred to as Geometric Conservation Law (GCL) and it is equivalent to the local kinematic equation which governs the mesh motion:

$$
\frac{\mathrm{d} \mathbf{x}}{\mathrm{d} t}=\mathbf{v}, \quad \mathbf{x}(t=0)=\mathbf{x}_{0}
$$

where $\mathbf{x}_{0}$ denotes the initial position at time $t=0$ of the material particle. The velocity gradient operator $\mathbb{L}(\mathbf{v})$ used in (28e) is defined by applying the divergence theorem as

$$
\mathbb{L}(\mathbf{v})=\nabla \mathbf{v}=\frac{1}{|\Omega|} \int_{\partial \Omega} \mathbf{v} \otimes \mathbf{n} \mathrm{d} s
$$

which is the cornerstone for a compatible space discretization with the geometric conservation law (28a).

The above integral formulation (28) of the GPR model is suitable for the design of cell-centered finite volume methods.

\subsection{Fully discrete first order scheme}

Equation (26) implies that the mass of a generic control volume remains constant in time, that is

$$
m_{i}:=\int_{T_{i}(t)} \rho \mathrm{d} \mathbf{x},
$$

with $m_{i}$ denoting the mass of cell $T_{i}(t)$ over time. As usual for finite volume schemes, data are stored and evolved in time as piecewise constant cell averages. Therefore, for a generic variable $\phi(\mathbf{x}, t)$, its mass averaged value $\phi_{i}$ over the element $T_{i}(t)$ is defined as

$$
\phi_{i}=\frac{1}{m_{i}} \int_{T_{i}(t)} \rho \phi \mathrm{d} \mathbf{x} .
$$

Following [81], let us introduce the subcell force $\boldsymbol{f}_{r i}$, which is the traction force exerted on the outer boundary of the subcell $T_{r i}$ (see Figure 1). Specifically, the surface integral of the momentum equation (28b) is split over the subcell boundaries, hence obtaining

$$
\int_{\partial T_{i}} \boldsymbol{T n} \mathrm{d} s=\sum_{r \in \mathcal{R}_{i}} \int_{\partial T_{r i} \cap \partial T_{i}} T \mathbf{n} \mathrm{d} s,
$$

which yields the definition of the subcell force [81, 82] as

$$
f_{r i}:=\int_{\partial T_{r i} \cap \partial T_{i}} \operatorname{Tn} \mathrm{d} s .
$$

Using the definition of the subcell force and the mass average quantity introduced above, a fully discrete finite volume scheme for system (28) writes

$$
\begin{aligned}
& \omega_{i}^{n+1}=\omega_{i}^{n}+\frac{\Delta t}{m_{i}} \sum_{r \in \mathcal{R}_{i}} \tilde{\mathbf{v}}_{r}^{*} \cdot \frac{1}{6}\left(\mathbf{c}_{r i}^{n}+4 \mathbf{c}_{r i}^{n+1 / 2}+\mathbf{c}_{r i}^{n+1}\right), \\
& \mathbf{v}_{i}^{n+1}=\mathbf{v}_{i}^{n}+\frac{\Delta t}{m_{i}} \sum_{r \in \mathcal{R}_{i}} \tilde{\boldsymbol{f}}_{r i}^{*}, \\
& E_{i}^{n+1}=E_{i}^{n}+\frac{\Delta t}{m_{i}}\left[\sum_{r \in \mathcal{R}_{i}} \tilde{\boldsymbol{f}}_{r i}^{*} \cdot \tilde{\mathbf{v}}_{r}^{*}+\sum_{f \in \mathcal{F}_{i}} \widehat{\mathbf{q}_{f i} \cdot \mathbf{n}_{f i}}{ }^{n} s_{f}^{n}\right]=0, \\
& \mathbf{J}_{i}^{n+1}=\mathbf{J}_{i}^{n}-\frac{\Delta t}{m_{i}} \sum_{f \in \mathcal{F}_{i}} \widehat{T_{f i} \boldsymbol{I} \cdot \mathbf{n}_{f i}}{ }^{n} s_{f}^{n}-\Delta t \frac{\mathbf{H}_{i}^{n+1}}{\Psi_{i}^{n+1}}, \\
& \mathbf{G}_{e_{i}}^{n+1}=\mathbf{G}_{e_{i}}^{n}-\Delta t\left(\mathbf{G}_{e_{i}}^{n} \mathbb{L}_{i}\left(\tilde{\mathbf{v}}^{*}\right)+\mathbb{L}_{i}\left(\tilde{\mathbf{v}}^{*}\right)^{\top} \boldsymbol{G}_{e_{i}}^{n}\right)+\Delta t \frac{2 \boldsymbol{\sigma}_{i}^{n+1}}{\rho \Theta_{i}^{n+1}},
\end{aligned}
$$


which must be coupled with the discrete trajectory equation to move the grid nodes:

$$
\mathbf{x}_{r}^{n+1}=\mathbf{x}_{r}^{n}+\Delta t \tilde{\mathbf{v}}_{r}^{*} .
$$

The vector of state variables is $\mathbf{Q}=\left\{\omega, \mathbf{v}, E, \mathbf{J}, \boldsymbol{G}_{e}\right\}$, and these quantities are evolved in time with the discrete equations written above. Let us now examine more in details the finite volume scheme (35).

Time discretization. All terms in (35) are discretized explicitly, apart from the sources in the thermal impulse equation (35d) and in the equation for the metric tensor (35e), because they might become stiff when approaching the limit cases of Navier-Stokes and Fourier. The stiff source terms are handled with an exponential integrator for ODE that will be presented in Section 3.5, which is applied to both the thermal impulse (35d) and the metric tensor equations (35e). Consequently, an implicit discretization of $\boldsymbol{G}_{e_{i}}^{n+1}$ implies that the stress tensor $\boldsymbol{\sigma}_{i}^{n+1}$ is also taken implicitly. Therefore a formally implicit treatment is considered for the total stresses in the momentum and energy equations (35b) and (35c), embedded in the subcell force $\boldsymbol{f}_{r i}$ according to (34), as well as for the node velocity. These terms are marked with the asterisk superscript, i.e. $\tilde{\boldsymbol{f}}_{r i}^{*}$ and $\tilde{\mathbf{v}}_{r}^{*}$. To overcome the resulting non-linearity in the stress tensor, i.e. $\boldsymbol{\sigma}_{i}^{n+1}=\boldsymbol{\sigma}_{i}^{n+1}\left(\boldsymbol{G}_{e_{i}}^{n+1}\right)$ according to (4), an iterative Picard technique will be introduced later for the computation of the node velocity and

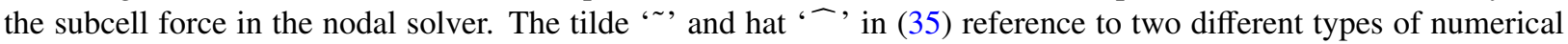
fluxes explained below.

According to (36), the coordinate position $\mathbf{x}_{r}$ is a linear function of time. The corner vector $\mathbf{c}_{r i}$ in (35a) exhibits a linear or quadratic time dependency, in $2 \mathrm{D}$ or $3 \mathrm{D}$, respectively. Therefore, the flux in the equation for the specific volume $\omega_{i}^{n+1}$ must be exactly integrated in time in order to ensure the satisfaction of the GCL at the discrete level. In (35a), the fourth order accurate Kepler quadrature rule is used, which might be replaced by a simple midpoint rule for 2D meshes. This ensures that the new density $\rho_{i}^{n+1}$ computed from the GCL (35a) is equivalent to the density deduced from the mesh motion, i.e. $\rho_{i}^{n+1}=m_{i} /\left|T_{i}^{n+1}\right|$, see $[79,84]$ for further details.

Numerical fluxes. The finite volume scheme (35) involves two different types of numerical fluxes for the discretization of the divergence operators, namely vertex-based and face-based fluxes. The computation of the nodal velocity is based on the nodal solver presented in Section 3.4, where multiple one-dimensional Riemann problems are simultaneously solved across all faces impinging to a node. Numerical dissipation is embedded into the nodal solver, thus the resulting node velocity $\tilde{\mathbf{v}}_{r}^{*}$ is numerically stable. This velocity is subsequently used to compute the subcell force $\tilde{f}_{r i}^{*}$, and the tilde symbol in (35) indicates these vertex-based numerical fluxes.

The second type of fluxes are face-based and those terms are marked with the hat symbol. A Rusanov-type numerical flux function [99] is adopted, thus the heat flux in the energy equation (35c) and the temperature gradient in the thermal impulse equation (35d) are explicitly given by

$$
\begin{aligned}
\widehat{\mathbf{q}_{f i} \cdot \mathbf{n}_{f i}} & =\frac{1}{2}\left(\left(\alpha^{2} T \mathbf{J}\right)_{f i}+\left(\alpha^{2} T \mathbf{J}\right)_{f j}\right) \cdot \mathbf{n}_{f i}-\frac{1}{2}\left|\lambda_{f}\right|\left(E_{f j}-E_{f i}\right), \\
\widehat{T_{f i} \boldsymbol{I} \cdot \mathbf{n}_{f i}} & =\frac{1}{2}\left((T \boldsymbol{I})_{f i}+(T \boldsymbol{I})_{f j}\right) \cdot \mathbf{n}_{f i}-\frac{1}{2}\left|\lambda_{f}\right|\left(\mathbf{J}_{f j}-\mathbf{J}_{f i}\right),
\end{aligned}
$$

where the subscripts $f i$ and $f j$ denote the value of the physical quantity computed at face $f$ from element $T_{i}$ and from the neighbor element $T_{j}$, respectively. The numerical dissipation is given in terms of the maximum eigenvalue estimate $\lambda_{f}$ in spatial normal direction between the elements sharing face $f$, which is evaluated as

$$
\lambda_{f}=\max \left(a_{i}, a_{j}\right),
$$

with the wave speeds $\left(a_{i}, a_{j}\right)$ defined by (19).

Non-conservative products. The last equation (35e) contains non-conservative products between the metric tensor $\boldsymbol{G}_{e}$ and the velocity gradient $\mathbb{L}=\nabla \mathbf{v}$. This equation can be also seen as a pure geometric relation and thus, according to [27, 23], we do not consider the jump contribution across the cell boundaries of the non-conservative products as done in [19], but only the smooth part in the cell volume is taken into account. The discrete version of the velocity gradient (30) writes

$$
\mathbb{L}_{i}(\mathbf{v})=\frac{1}{\left|T_{i}\right|} \sum_{r \in \mathcal{R}(i)} \mathbf{v}_{r} \otimes \mathbf{c}_{r i},
$$


which is used to evaluate the products $\boldsymbol{G}_{e_{i}}^{n} \mathbb{L}_{i}\left(\tilde{\mathbf{v}}^{*}\right)$ and $\mathbb{L}_{i}\left(\tilde{\mathbf{v}}^{*}\right)^{\top} \boldsymbol{G}_{e_{i}}^{n}$ in (35e). Let us notice that the discrete gradient operator (40) is applied to the velocity field $\tilde{\mathbf{v}}^{*}$, which results from the nodal solver.

\subsection{Nonlinear nodal solver}

The nodal fluxes are computed starting from the nodal solver algorithm proposed in a series of works [83, 81, 80, $84,31]$. The nodal solver aims at evaluating a unique velocity vector at each node $\tilde{\mathbf{v}}_{r}^{*}$ by satisfying global conservation of momentum as well as compatibility with the second law of thermodynamics, meaning that the entropy variation in the cell is non-negative.

The starting point is the knowledge of the state variables $\mathbf{Q}^{n}$ and the geometry $\mathbf{x}^{n}$ at time $t^{n}$. Next, the nodal velocity $\tilde{\mathbf{v}}_{r}^{*}$ is obtained by solving the system

$$
\boldsymbol{M}_{r} \tilde{\mathbf{v}}_{r}^{*}=\sum_{i \in \mathcal{T}_{r}} \boldsymbol{M}_{i r} \mathbf{v}_{i}^{n}-\mathbf{T}_{i}^{*} \mathbf{c}_{r i}^{n},
$$

with the discrete subcell matrix $\boldsymbol{M}_{i r}$ and nodal matrix $\boldsymbol{M}_{r}$ given by

$$
\boldsymbol{M}_{i r}=\sum_{f \in \mathcal{F}_{r i}} z_{i}^{n} s_{f}^{n} \mathbf{n}_{f}^{n} \otimes \mathbf{n}_{f}^{n}, \quad \boldsymbol{M}_{r}=\sum_{i \in \mathcal{T}_{r}} \boldsymbol{M}_{i r} .
$$

The numerical dissipation is introduced by the swept mass flux $z_{i}^{n}=\rho_{i}^{n} a_{i}^{n}$, with $a_{i}^{n}$ given by (19). Notice that the above matrices are symmetric positive definite by construction, thus $\boldsymbol{M}_{r}$ in (41) is always invertible. Once the node velocity is computed, the subcell force can easily be evaluated as

$$
\tilde{f}_{r i}^{*}=\mathbf{c}_{r i}^{n} \boldsymbol{T}_{i}^{*}+\boldsymbol{M}_{i r}\left(\tilde{\mathbf{v}}_{r}^{*}-\mathbf{v}_{i}^{n}\right),
$$

whereas the node coordinates are updated to the new position with the trajectory equation (36).

The geometric quantities $\mathbf{c}_{r i}^{n}$ and $\mathbf{n}_{f}^{n}$ are considered at the current time level $t^{n}$ as well as the cell quantities $\mathbf{v}_{i}^{n}$ and $z_{i}^{n}$, which are evaluated at the cell centroid, i.e. $\mathbf{v}_{r}\left(\mathbf{x}_{i}\right)^{n}$ and $z_{i}\left(\mathbf{x}_{i}\right)^{n}$. However, the total Cauchy stress $\boldsymbol{T}_{i}^{*}$, and more specifically the tangential stress tensor $\sigma_{i}^{*}$, should take into account elastic, viscous or plasticity effects that might occur in the material, yielding a significant change in the node velocity. Therefore, a formally implicit discretization is used for the tangential stress tensor, that according to (4) leads to (for the energy potential (3))

$$
\boldsymbol{\sigma}_{i}^{n+1}=-\rho_{i}^{n+1} c_{\mathrm{sh}}^{2} \mathbf{G}_{e_{i}}^{n+1}{\stackrel{\mathbf{G}}{e_{i}}}^{n+1},
$$

so that the nodal solver (41) must be coupled with the trajectory equation (36), the GCL (35a) and the equation for $\mathbf{G}_{e_{i}}$ (35e), in order to obtain $\mathbf{x}_{r}^{n+1}, \rho_{i}^{n+1}$ and $\mathbf{G}_{e_{i}}^{n+1}$, respectively. This choice wold lead to a strongly nonlinear system to be solved, whose convergence would become difficult to control. Consequently, we rely on a Picard iterative technique that has been already used in the context of all Mach flow solvers [16, 17]. More precisely, let $l$ be the index for the Picard iteration, and let the viscous stress tensor be initialized at time $t^{n}$, i.e. $\sigma_{i}^{l, n+1}=\sigma_{i}^{n}$ for $l=0$. The nodal solver algorithm proceeds then iteratively as follows for $l=1, \ldots, \mathcal{L}$ :

$$
\begin{aligned}
& \tilde{\mathbf{v}}_{r}^{l+1, n+1}=\left(\sum_{i \in \mathcal{T}_{r}} \boldsymbol{M}_{i r} \mathbf{v}_{i}^{n}-\boldsymbol{T}_{i}^{l, n+1} \mathbf{c}_{r i}^{n}\right) \boldsymbol{M}_{r}^{-1}, \\
& \mathbf{x}_{r}^{l+1, n+1}=\mathbf{x}_{r}^{n}+\Delta t \tilde{\mathbf{v}}_{r}^{l+1, n+1}, \\
& \rho_{i}^{l+1, n+1}=\frac{m_{i}}{\left|T_{i}\right|^{l+1, n+1},} \\
& \mathbf{G}_{e_{i}}^{l+1, n+1}=\mathbf{G}_{e_{i}}^{n}-\Delta t\left(\mathbf{G}_{e_{i}}^{n} \mathbb{L}_{i}\left(\tilde{\mathbf{v}}^{l+1, n+1}\right)+\mathbb{L}_{i}\left(\tilde{\mathbf{v}}^{l+1, n+1}\right)^{\top} \mathbf{G}_{e_{i}}^{n}\right)+\Delta t \frac{2 \boldsymbol{\sigma}_{i}^{l+1, n+1}}{\rho^{l+1, n+1} \Theta_{i}^{l+1, n+1}}, \\
& \boldsymbol{\sigma}_{i}^{l+1, n+1}=-\rho_{i}^{l+1, n+1} c_{\mathrm{sh}}^{2} \mathbf{G}_{e_{i}}^{l+1, n+1}{\stackrel{\mathbf{G}}{e_{i}}}^{l+1, n+1} .
\end{aligned}
$$

The only fully implicit discretization is concerned with the computation of $\mathbf{G}_{e_{i}}^{l+1, n+1}$, where the non-linearity contained in the source term is solved by an exponential integrator discussed in the next section. To improve the efficiency of 
the iterative scheme (45), the new density $\rho_{i}^{l+1, n+1}$ is deduced from the new volume $\left|T_{i}\right|^{l+1, n+1}$ computed with the new coordinates $\mathbf{x}_{r}^{l+1, n+1}$, thus the GCL (35a) is not directly solved. However, due to the compatible time update of the fluxes in (35a), this approach is equivalent to explicitly solve the GCL. The multi-index $\{l, n+1\}$ is shorten to the asterisk index $\{*\}$, hence the result of the nonlinear nodal solver is the nodal velocity $\tilde{\mathbf{v}}_{r}^{*}$ and the subcell force $\tilde{\boldsymbol{f}}_{r i}^{*}$, evaluated according to (43), with the total Cauchy stress $\boldsymbol{T}_{i}^{*}$ (which is a function of $\boldsymbol{\sigma}_{i}^{*}$ ) computed within the Picard loop (45). The iterative scheme stops when one of the following exit conditions is satisfied, based on a tolerance set to $\delta=10^{-12}$.

- The material is an ideal gas, thus the hydrodynamics limit of the model is reached:

$$
\epsilon_{h}^{l+1}:=\left|\boldsymbol{G}_{e_{i}}^{l+1, n+1}-\left(\frac{\rho^{l+1, n+1}}{\rho_{0}}\right)^{2 / 3} \boldsymbol{I}\right| \leq \delta,
$$

which corresponds to the stiff relaxation limit for $\mathbf{G}_{e}$.

- The material is a purely elastic solid, therefore

$$
\epsilon_{e}^{l+1}:=\left|\mathbf{G}_{e_{i}}^{l+1, n+1}-\mathbf{G}_{i, *}^{l+1, n+1}\right| \leq \delta,
$$

where $\boldsymbol{G}_{i, *}^{l+1, n+1}$ is the solution of the homogeneous equation related to (45d). This condition means that the source term vanishes and no relaxation is needed.

- Convergence is achieved between two consecutive iterations for any of the following residuals:

$$
\left|\epsilon_{h}^{l+1}-\epsilon_{h}^{l}\right| \leq \delta, \quad\left|\epsilon_{e}^{l+1}-\epsilon_{e}^{l}\right| \leq \delta
$$

The maximum number of iterations is set to $\mathcal{L}=10$. In the numerical tests shown in Section 5 , the nonlinear nodal solver has always achieved convergence before reaching $\mathcal{L}$ iterations. Furthermore, let us point out that if the material is either an ideal fluid or an elastic solid, the nonlinear solver converges in one Picard iteration, namely one of the exit conditions between (46) and (47) is fulfilled. In these particular situations, the discretization of the tangential stresses $\sigma$ becomes fully explicit and the nodal solver exactly reduces to the EUCCLHYD scheme [79] for ideal hydrodynamics, or the corresponding version for hyperelasticity materials presented in [27, 23].

Remark. Boundary conditions are imposed in a compatible way with our numerical scheme. More precisely, either the Cauchy stress or the velocity normal to a boundary face must be prescribed in the nodal solver in order to ensure global conservation of momentum. All the details can be found in [83, 80, 22, 23]. The face-based fluxes are computed with a standard finite volume scheme that requires ghost states to properly set the boundary condition. The reader is referred to [15] and references therein for an exhaustive explanation on the treatment of boundary conditions in cell-centered finite volume schemes on moving unstructured meshes.

\subsection{Exponential integrator for stiff source terms}

Due to the wide span of possible timescales characterizing the evolution of the elastic metric tensor $\boldsymbol{G}_{e}$, the strain relaxation source on the right hand side of Eq. (2e) oftentimes is of stiff nature and cannot be easily treated with an explicit method. The same holds for the simpler thermal impulse equation (2d). For this reason we employ an efficient semi-implicit, semi-analytical integration scheme that can handle such arbitrarily stiff source terms, and in particular can accurately recover the asymptotic equilibrium state associated with viscous fluxes in the Navier-Stokes equations and also relax to the Fourier limit of thermal conduction.

\subsubsection{Thermal impulse relaxation solver}

The thermal impulse equation (2d) can be written in extended form as

$$
\frac{\mathrm{d} \mathbf{J}}{\mathrm{d} t}=-\frac{\nabla T}{\rho}-\frac{\alpha^{2} T}{\rho \kappa} \mathbf{J}=\mathbf{P}_{*}-\frac{1}{\tau_{2}} \mathbf{J},
$$


which, once the discretization of $-\nabla T / \rho$ is fixed (to the constant value $\mathbf{P}_{*}$ ), can be seen as a system of three uncoupled first order linear ordinary differential equations (ODEs) and an exact solution is indeed found thanks to the linearity and independence of the three equations. Explicitly, the solution is

$$
\mathbf{J}^{n+1}=\left(\mathbf{J}^{n}-\tau_{2} \mathbf{P}_{*}\right) \exp \left(-\Delta t / \tau_{2}\right)+\tau_{2} \mathbf{P}_{*}
$$

with $\mathbf{P}_{*}=-\sum_{f \in \mathcal{F}_{i}} \widehat{T_{f i} \boldsymbol{I} \cdot \mathbf{n}_{f i}}{ }^{n} s_{f}^{n} / m_{i}$ being approximated as the constant discrete time-derivative of $\mathbf{J}$ as given by the update formula (35d) when the source term is neglected. The only degenerate case to be considered is that if $\Delta t / \tau_{2}$ is very small (of the order of $10^{-8}$ ), i.e. if the source term is not stiff at all, then (50) might yield inaccurate results, due to floating point representation issues. In this case, the solution algorithm simply opts to switch to explicit Euler integration, which for such mild (vanishing) sources yields perfectly valid solutions.

Finally the result can be combined with the implicit-explicit Runge-Kutta framework by separating the (formally implicit) contribution due to the relaxation source $\Delta \mathbf{J}_{r e l}=\mathbf{J}^{n+1}-\left(\mathbf{J}^{n}+\Delta t \mathbf{P}_{*}\right)$ from the explicit operator associated with the convective discretization $\Delta \mathbf{J}_{\text {conv }}:=\Delta t \mathbf{P}_{*}$.

\subsubsection{Strain relaxation solver}

The solver for the strain relaxation source is based on the exponential integrator developed in [107] for the computation of diffuse interface fractures and material failure, but exploits in a deeper manner the particular structure of the equation being solved, following the technique presented in [36] for the integration of stiff finite-rate pressure relaxation sources.

An important aspect of the scheme is that it avoids fractional-step-type splitting, so that the Navier-Stokes stress tensor and the Fourier heat flux can be recovered regardless of the ratio between the computational time step size and the relaxation timescales. This means that the global time step size need not to be adjusted to accommodate for the fast dynamics of the relaxation sources.

Moreover, we recall that the solver employed in [107] required, in general, the solution of a sequence of a nonhomogeneous nine-by-nine systems of linear ordinary differential equations for the nine independent components of the distortion field $\boldsymbol{A}_{e}$, which involves the numerical computation of matrix exponentials and the inversion of the Jacobian matrix of the ODE system. Both these operations constitute delicate tasks in linear algebra that require special care to be carried out in an efficient and accurate manner.

The approach used in this work entirely foregoes the solution of such nine-by-nine systems (six-by-six, in the case of the symmetric tensor $\mathbf{G}_{e}$ ) and the associated linear algebra intricacies. Thus, we compute the analytical solution to one of the several different linearized equations (automatically chosen by the solver) that approximate the nonlinear ODE

$$
\frac{\mathrm{d} \mathbf{G}_{e}}{\mathrm{~d} t}=\boldsymbol{L}_{*}-\frac{6}{\tau_{1}} \operatorname{det}\left(\mathbf{G}_{e}\right)^{5 / 6} \mathbf{G}_{e} \dot{\mathbf{G}}_{e},
$$

while admitting simple solutions that can be evaluated in a robust fashion. Here, with $\boldsymbol{L}_{*}$ we denote a constant convective forcing term to be given in the following paragraphs, in analogy to the previously defined $\mathbf{P}_{*}$ discrete time derivative of thermal impulse.

This is achieved by first computing, cell by cell, the update to $\mathbf{G}_{e}$ associated with the left hand side of (2e), and then including its effects in (51), in the form of the constant forcing term $\boldsymbol{L}_{*}$. Formally, this first step amounts to computing the solution $\mathbf{G}_{e *}=\mathbf{G}_{e}\left(t_{n+1}\right)$ to the initial value problem

$$
\left\{\begin{array}{l}
\frac{\mathrm{d} \mathbf{G}_{e}}{\mathrm{~d} t}+\mathbf{G}_{e} \nabla \mathbf{v}+\nabla \mathbf{v}^{\top} \mathbf{G}_{e}=\mathbf{0}, \\
\mathbf{G}_{e}\left(t^{n}\right)=\mathbf{G}_{e}{ }^{n},
\end{array}\right.
$$

which, in our case, is solved with the compatible method applied to the equation for the metric tensor $\boldsymbol{G}_{e}$, yielding a point-wise update to the cell averages that allows to define the constant convective forcing term

$$
\boldsymbol{L}_{*}=\frac{\mathbf{G}_{e *}-\mathbf{G}_{e}{ }^{n}}{\Delta t} .
$$

Then a sub-timestepping loop with adaptive step size $\delta t^{m}$ is entered in order to approximate the solution of (51) with a sequence of solutions of linearized ODEs. Such a sub-timestepping loop is useful for ensuring the robustness 
and accuracy of the solver in complex flow configurations, but generally in our computations the solver achieves convergence in one single sub-time-step $\delta t^{m}=\Delta t$. We remand to [36, 107] for more details on the sub-timestepping approach, and we carry on our presentation of the method by listing three possible approximation choices for the solution of (51).

\subsubsection{Approximate analytical solution for strain (1)}

When dealing with fluid flows (i.e., when the source term acts on fast timescales), rather often one may assume that $\mathbf{G}_{e}$ is a perturbation of a spherical tensor, that is, $\stackrel{\mathbf{G}}{e}_{e}$ can be assumed small. Then, it is advantageous to rewrite the evolution equation for the elastic metric tensor (51) as

$$
\frac{\mathrm{d} \boldsymbol{G}_{e}}{\mathrm{~d} t}=\boldsymbol{L}_{*}-k \dot{\boldsymbol{G}}_{e} \dot{\mathbf{G}}_{e}+k\left(\frac{\operatorname{tr} \mathbf{G}_{e}}{3}\right)^{2} \boldsymbol{I}-k \frac{\operatorname{tr} \boldsymbol{G}_{e}}{3} \mathbf{G}_{e},
$$

with $k=6 \operatorname{det}\left(\boldsymbol{G}_{e}\right)^{5 / 6} / \tau_{1}$ taken constant for the sub-time-step. This splits the source in four pieces. The first is the constant $\boldsymbol{L}_{*}$, associated with convection which, by definition, cannot be stiff as its size is limited by the CFL constraint of the global timestepping scheme. The second is a (small by hypothesis) quadratic term in $\dot{G}_{e}$ which can be safely approximated as constant. The third is a function of $\operatorname{tr} \boldsymbol{G}_{e}$ only, again formally taken constant. This assumption can be justified by writing the evolution equation for the trace of the metric tensor $\mathbf{G}_{e}$

$$
\frac{\mathrm{d}}{\mathrm{d} t}\left(\operatorname{tr} \mathbf{G}_{e}\right)=\boldsymbol{L}_{*}-k \operatorname{tr}\left(\stackrel{\circ}{e}_{e} \stackrel{\circ}{e}_{e}\right)
$$

which shows that either $\operatorname{tr} G_{e}$ varies on a timescale associated with convection (by definition, slow), or as a quadratic function of $\boldsymbol{G}_{e}$ (small by assumption). The approximate equation (54) then admits the simple exact solution

$$
\mathbf{G}_{e}^{m+1}=\mathbf{G}_{e}\left(t^{m}+\delta t^{m}\right)=\exp \left(-k \frac{\operatorname{tr} \boldsymbol{G}_{e}}{3} \delta t^{m}\right)\left(\mathbf{G}_{e}{ }^{m}+\boldsymbol{F}_{0}\right)-\boldsymbol{F}_{0},
$$

with

$$
\boldsymbol{F}_{0}=-\frac{3}{k \operatorname{tr} \mathbf{G}_{e}}\left(\boldsymbol{L}_{*}+k\left(\frac{\operatorname{tr} \boldsymbol{G}_{e}}{3}\right)^{2} \boldsymbol{I}-k \stackrel{\circ}{e}_{e} \stackrel{\mathbf{G}}{e}_{e}\right) .
$$

We should remark that nowhere in this approximate solution we neglected the contributions due to $\mathbf{G}_{e}$, they only have taken to be constant for a sub-time-step, so that, in principle there are no hypotheses restricting the use of such an approximation, beside increased requirements imposed on the sub-timestepping scheme when $\boldsymbol{G}_{e}$ is not small. Specifically, in this work our constant approximations are initially set to $\operatorname{tr} \mathbf{G}_{e}=\operatorname{tr} \mathbf{G}_{e}{ }^{m}$ and $\mathbf{G}_{e}=\dot{\mathbf{G}}_{e}{ }^{m}$ and then updated within a fixed point iteration as $\operatorname{tr} \mathbf{G}_{e}=\operatorname{tr}\left(\mathbf{G}_{e}{ }^{m}+\mathbf{G}_{e}{ }^{m+1}\right) / 2$ and $\mathbf{G}_{e}=\operatorname{dev}\left(\mathbf{G}_{e}{ }^{m}+\mathbf{G}_{e}{ }^{m+1}\right) / 2$.

\subsubsection{Approximate analytical solution for strain (2)}

Whenever the deviatoric part of $\mathbf{G}_{e}$ cannot be assumed small, i.e. in practice when

$$
\sqrt{\operatorname{tr}\left(\dot{\boldsymbol{G}}_{e}^{m}{\stackrel{\circ}{\mathbf{G}_{e}}}^{m}\right)}>\epsilon_{1} \operatorname{det}\left(\mathbf{G}_{e}{ }^{m}\right)^{1 / 3},
$$

better accuracy in the approximation of (51) can be obtained by observing that it is possible to switch the order of the operands of the matrix product $\mathbf{G}_{e} \mathbf{G}_{e}$ appearing in (51). In this work the coefficient $\epsilon_{1}$ is permanently set to $\epsilon_{1}=0.2$, which means that this approximation mode has been used also when $\boldsymbol{G}_{e}$ is not strictly small and indeed one may in principle choose higher values for $\epsilon_{1}$ without compromising the behavior of the solver. Then we can rewrite (51) as

$$
\frac{\mathrm{d} \mathbf{G}_{e}}{\mathrm{~d} t}=\boldsymbol{L}_{*}-k \mathbf{G}_{e} \stackrel{\circ}{\mathbf{G}}_{e}
$$

where we will take $\boldsymbol{L}_{*}, k=6 \operatorname{det} \boldsymbol{G}_{e}{ }^{5 / 6} / \tau$, and $\dot{\boldsymbol{G}}_{e}$ to be constant at each sub-time-step. At the implementation level, in order to simplify the solution of (59), we work in the principal reference frame, which diagonalizes $\boldsymbol{G}_{e}$ and $\boldsymbol{G}_{e}$, i.e. 
we compute the orthonormal matrix $\boldsymbol{E}$ such that $\hat{\boldsymbol{G}}_{e}=\boldsymbol{E}^{-1} \boldsymbol{G}_{e} \boldsymbol{E}$ and $\hat{\mathbf{G}}_{e}=\boldsymbol{E}^{-1} \stackrel{\circ}{\mathbf{G}}_{e} \boldsymbol{E}$ are diagonal matrices and apply the associated change of basis to all vectors and tensors in our equation. In this way the exact solution to Eq. (59) is

$$
\boldsymbol{G}_{e}{ }^{m+1}=\mathbf{G}_{e}\left(t^{m}+\delta t^{m}\right)=\boldsymbol{E}\left[\exp \left(-k \hat{\boldsymbol{G}}_{e} \delta t^{m}\right)\left(\boldsymbol{E}^{-1} \boldsymbol{G}_{e}{ }^{n}+\frac{1}{k} \hat{\boldsymbol{G}}_{e}^{-1} \boldsymbol{E}^{-1} \boldsymbol{L}_{*}\right)-\frac{1}{k} \hat{\boldsymbol{G}}_{e}^{-1} \boldsymbol{E}^{-1} \boldsymbol{L}_{*}\right]
$$

The three-by-three matrix $\boldsymbol{E}$ having for columns the eigenvectors of $\boldsymbol{G}_{e}$ can be quickly and robustly computed to arbitrary precision by means of Jacobi method for the eigenstructure of symmetric matrices, and its inverse is simply given by $\boldsymbol{E}^{-1}=\boldsymbol{E}^{\top}$. Furthermore, $\hat{\boldsymbol{G}}_{e}$ can be inverted trivially in the principal reference frame by just taking the reciprocal of each diagonal entry. Like for the previous solution we iteratively update the constant estimate $\hat{\mathbf{G}}_{e}=$ $\operatorname{dev}\left(\hat{\boldsymbol{G}}_{e}{ }^{m}+\hat{\boldsymbol{G}}_{e}{ }^{m+1}\right) / 2$ so to gain higher accuracy while maintaining the linearity of the ODE being solved.

\subsubsection{Determinant constraint}

In the solution of the equation for the metric tensor $\mathbf{G}_{e}$, specifically when the computation involves fluid-type behavior, special care must be paid to preserve the nonlinear algebraic constraint $\operatorname{det} \mathbf{G}_{e}(t, \mathbf{x})=\left(\rho(t, \mathbf{x}) / \rho_{0}\right)^{2}$. For the purpose of notation compactness, in the following we will denote the target determinant as $D(t, \mathbf{x})=\left(\rho(t, \mathbf{x}) / \rho_{0}\right)^{2}$. Thanks to the compatible discretization of the scheme presented in this work, the GCL is fulfilled, and the solution for density is evolved in a way that is compatible with the mesh motion, thus the convective part of the equation satisfies the determinant constraint up to the order of accuracy of the time discretization (see [23] for further details). However, the same constraint must be actively enforced when source terms are present. A simple approach to the problem consists in uniformly multiplying all components of $\mathbf{G}_{e}$ by $\left(D / \operatorname{det} \mathbf{G}_{e}\right)^{1 / 3}$ so that the resulting determinant will be $D$.

The specific numerical value of the target determinant $D$ is clearly known (as a function of density) at the time levels $t^{n}$ and $t^{n+1}$, however it must be somehow approximated for all the in-between times during which we operate our sub-timestepping procedure. In this work we impose that for a given sub-time-step indexed by $m$, connecting $t^{m}$ and $t^{m+1}$, the determinant $D$ is computed as

$$
D=\beta_{s} D_{s}+\left(1-\beta_{s}\right) D_{f}
$$

where we define $D_{s}=\operatorname{det}\left(\boldsymbol{G}_{e}+\left(t^{m}+\delta t^{m}-t^{n}\right) \boldsymbol{L}_{*}\right)$ to be the value that the determinant would have following a linear segment path connecting the two states $\boldsymbol{G}_{e}$ and $\boldsymbol{G}_{e_{*}}$, that is, the value that would allow exact integration of the (zero) source term in the solid limit to be preserved. In the fluid limit instead, we take $D_{f}=\operatorname{det} \mathbf{G}_{e}+\left(t^{m}+\delta t^{m}-t^{n}\right)\left(\operatorname{det} \boldsymbol{G}_{e_{*}}-\right.$ $\operatorname{det} \boldsymbol{G}_{e}{ }^{n}$ ) as a second order approximation of the determinant. The mixing ratio $\beta_{s}$ for the two approximations $D_{s}$ and $D_{f}$ is a heuristic measure of how close to a solid can the material be considered and in particular the expression we adopt is

$$
\beta_{s}=\min \left(1, \frac{\left\|\boldsymbol{L}_{*}\right\|_{2}^{2}}{\left\|6 / \tau_{1} \operatorname{det}\left(\mathbf{G}_{e}^{m}\right)^{5 / 6} \mathbf{G}_{e}{ }^{m} \stackrel{\mathbf{G}}{e}^{m}\right\|_{2}^{2}+10^{-14}}\right)^{4}
$$

with $\|\bullet\|_{2}^{2}$ denoting the square of the Frobenius norm of a given tensor.

\subsubsection{Approximate analytical solution for strain (3): fixed point iteration for the Navier-Stokes equilibrium state}

Oftentimes the timescale $\tau_{1}$ of strain relaxation is so fast that one may decide, out of computational efficiency concerns, to just compute the strain state for which the forcing term due to convection $\boldsymbol{L}_{*}$ and the relaxation source are balanced yielding a local equilibrium state corresponding to the Navier-Stokes limit of the GPR model. Such an equilibrium state can be easily computed by means of a fixed point iteration in the form

$$
\begin{aligned}
& \widetilde{\boldsymbol{G}}_{e l}=\frac{\tau_{1}}{6 \operatorname{det}\left(\mathbf{G}_{e l}\right)^{5 / 6}} \operatorname{dev}\left(\boldsymbol{G}_{e l}^{-1} \boldsymbol{L}_{*}\right)+\frac{\operatorname{tr}\left(\boldsymbol{L}_{*}-\boldsymbol{G}_{e l} \operatorname{dev}\left(\mathbf{G}_{e l}^{-1} \boldsymbol{L}_{*}\right)\right)}{\operatorname{tr}\left(\mathbf{G}_{e l}^{-1} \boldsymbol{L}_{*}\right)} \boldsymbol{I}, \\
& \boldsymbol{G}_{e l+1}=\widetilde{\boldsymbol{G}}_{e l}\left(\frac{D}{\operatorname{det} \widetilde{\boldsymbol{G}_{e l}}}\right)^{1 / 3},
\end{aligned}
$$


with $D$ the target determinant as defined in (61) and $l$ the iteration index in the fixed point procedure. We found that the fixed point iteration (63) is always convergent regardless of the initial guess, but nonetheless we care to provide a simple and efficient choice in the form

$$
\boldsymbol{G}_{e 1}=\widetilde{\boldsymbol{G}}_{e 0}\left(\frac{D}{\operatorname{det} \widetilde{\boldsymbol{G}}_{e 0}}\right)^{1 / 3} \text {, with } \widetilde{\boldsymbol{G}}_{e 0}=\boldsymbol{I}+\frac{\tau_{1}}{6 \operatorname{det}\left(\mathbf{G}_{e}{ }^{m}\right)^{5 / 6}} \operatorname{dev} \boldsymbol{L}_{*} .
$$

We remark that, when the relaxation timescale is much faster than the time step size, this approximate solution is not only very efficient and robust, but will also converge to the Navier-Stokes equilibrium state up to machine accuracy.

\subsubsection{Summary of the selection procedure for the approximation method}

At each sub-timestep between $t^{m}$ and $t^{m+1}$, our solver for the equation of the elastic metric tensor $\mathbf{G}_{e}$ has to select the optimal approximation method for the specific flow configuration at hand. The selection procedure is carried out as follows:

1. If the source is not stiff, i.e. in practice if $\beta_{s}>1-10^{-14}$, then we use explicit Euler integration and compute the solution at the next time sub-level as $\mathbf{G}_{e}{ }^{m+1}=\mathbf{G}_{e}{ }^{m}+\Delta t\left(\boldsymbol{L}_{*}-\frac{6}{\tau_{1} \operatorname{det}\left(\mathbf{G}_{e}{ }^{m}\right)^{5 / 6} \mathbf{G}_{e} \mathbf{G}_{e}}\right)$.

2. Else, we define the indicator matrix $\boldsymbol{\Lambda}=\operatorname{abs}\left(\mathbf{G}_{e}{ }^{m-1} \boldsymbol{L}_{*}-k \dot{\boldsymbol{G}}_{e}{ }^{m}\right)$ and if the sum of the off-diagonal components of $\Lambda$ is less than $\operatorname{tr} \Lambda / 5$ while also $\delta t^{m}>\tau_{1}$ holds, then the scheme opts for the fixed point iteration (63). Here, $\operatorname{abs}(\bullet)$ is applied component-wise to a given matrix.

3. Else, if $\sqrt{\operatorname{tr}\left(\dot{\boldsymbol{G}}_{e}^{m} \stackrel{\mathrm{G}}{e}^{m}\right)}<\epsilon_{1} \operatorname{det}\left(\mathbf{G}_{e}{ }^{m}\right)^{1 / 3}$ or if any of the diagonal entries of $\hat{\boldsymbol{G}}_{e}{ }^{m}$ has magnitude smaller than $\epsilon_{2} \operatorname{tr} \hat{\boldsymbol{G}}_{e}{ }^{m}$, then the scheme selects (54). In this work we permanently set $\epsilon_{2}=10^{-3}$ in order to prevent division by small numbers in (59).

4. If none of the above, then we apply approximation (59).

Regardless of the chosen approximation method, at the end of each sub-timestep, the result $\mathbf{G}_{e}{ }^{m+1}=\mathbf{G}_{e}\left(t^{m+1}\right)$ must be multiplied by $\left(D / \operatorname{det}\left(\mathbf{G}_{e}{ }^{m+1}\right)\right)^{1 / 3}$ so that the determinant constraint is satisfied.

\subsection{Second order extension in space and time}

The accuracy of the Godunov-type scheme (35) is improved up to second order in space and time by performing a spatial TVD linear reconstruction of the state variables and an IMEX Runge-Kutta time stepping scheme, respectively.

TVD piecewise linear reconstruction. To achieve second order of accuracy in space, a piecewise linear reconstruction is performed for all variables in the state vector $\mathbf{Q}$. The starting point is given by the known cell averages $\mathbf{Q}_{i}^{n}$ and the mesh configuration at time $t^{n}$. As a result, piecewise linear polynomials $\mathbf{w}_{i}^{n}\left(\mathbf{x}^{n}\right)$ are obtained for each cell and each variable of the governing system (2). This reconstruction polynomial is expressed in terms of a set of piecewise linear spatial basis functions that form a modal basis, that is

$$
\mathbf{w}_{i}^{n}\left(\mathbf{x}^{n}\right)=\sum_{l=1}^{\mathcal{M}} \psi_{l}(\boldsymbol{\xi}) \widehat{\mathbf{w}}_{l, i}^{n}:=\psi_{l}(\boldsymbol{\xi}) \widehat{\mathbf{w}}_{l, i}^{n},
$$

where the classical tensor index notation based on the Einstein summation convention is adopted, which implies summation over two equal indices. The modal basis functions $\psi_{l}(\boldsymbol{\xi})$ are defined in the reference system and count a total number $\mathcal{M}=d+1$ of unknown degrees of freedom. They explicitly write

$$
\psi_{l}(\xi)= \begin{cases}(1, \xi-1 / 3, \eta-1 / 3)^{\top} & \text { in 2D } \\ (1, \xi-1 / 4, \eta-1 / 4, \zeta-1 / 4)^{\top} & \text { in 3D }\end{cases}
$$

A so-called reconstruction stencil $\mathcal{S}_{i}=\bigcup_{j=1}^{n_{e}} T_{m(j)}^{n}$ is needed, where $1 \leq j \leq n_{e}$ is a local index that counts the elements belonging to the stencil, while $m(j)$ maps the local index to the global element numbers used in the mesh configuration 
(20). Due to the unstructured mesh, neither the stencil nor the element configuration is symmetric, thus the stencil contains a total number of $n_{e}=d \cdot \mathcal{M}$ elements to avoid ill-conditioned reconstruction matrices, as suggested in [3, 69]. The reconstruction stencil is filled considering the Voronoi neighbors of $T_{i}^{n}$ (i.e. the neighbor elements sharing at least one vertex with element $T_{i}^{n}$ ), that are recursively added until $n_{e}$ is reached. The reconstruction is based on integral conservation for each element $T_{j}^{n} \in \mathcal{S}_{i}$, that is

$$
\frac{1}{\left|T_{j}^{n}\right|} \int_{T_{j}^{n}} \psi_{l}(\boldsymbol{\xi}) \widehat{\mathbf{w}}_{l, i}^{c} \mathrm{~d} \mathbf{x}=\mathbf{Q}_{j}^{n}, \quad \forall T_{j}^{n} \in \mathcal{S}_{i},
$$

where the degrees of freedom $\widehat{\mathbf{w}}_{l, i}^{c}$ refer to the high order unlimited polynomial obtained from a central reconstruction. To enforce conservation of the reconstruction polynomial we must at least require that the above expression holds exactly for cell $T_{i}^{n}$, that is $\frac{1}{\left|T_{i}^{n}\right|} \int_{T_{i}^{n}} \psi_{l}(\boldsymbol{\xi}) \widehat{\mathbf{w}}_{l, i}^{c} \mathrm{~d} \mathbf{x}=\mathbf{Q}_{i}^{n}$. This linear constraint is added to the overdetermined system (67), which is solved only for the unknown expansion coefficients $\widehat{\mathbf{w}}_{l, i}^{c}$ relying on a classical Lagrangian multiplier approach, see $[19,45]$. The integrals appearing in (67) are evaluated with second order accurate quadrature rules and they are defined in the reference element $T_{e}$.

The above procedure generates a second order reconstruction polynomial within each cell, that is linear in the sense of Godunov [105], thus it is oscillatory and non-monotone across discontinuities of the numerical solution. To overcome this problem, the higher modes $\widehat{\mathbf{w}}_{l, i}^{c}$ of the expansion (65) must be limited so that the cell gradient respects the following monotonicity condition, which also ensures the TVD (Total Variation Diminishing) property:

$$
\mathbf{Q}_{i}^{n, \min } \leq \mathbf{w}_{i}^{n}\left(\mathbf{x}^{n}\right) \leq \mathbf{Q}_{i}^{n, \max }, \quad \mathbf{Q}_{i}^{n, \min }=\min \left\{\mathbf{Q}_{i}^{n}, \min _{j \in \mathcal{T}_{r(i)}} \mathbf{Q}_{j}^{n}\right\}, \quad \mathbf{Q}_{i}^{n, \max }=\max \left\{\mathbf{Q}_{i}^{n}, \max _{j \in \mathcal{T}_{r(i)}} \mathbf{Q}_{j}^{n}\right\},
$$

with $\mathcal{T}_{r(i)}$ denoting the set of elements which share at least one node with cell $T_{i}$. To respect the above relation (68), let us define a scalar $b_{i} \in[0 ; 1]$, that is used to limit the higher modes of the reconstruction polynomial. For each state variable and for $l=2, \ldots, \mathcal{M}$ we compute

$$
\widehat{\mathbf{w}}_{l, i}^{n}=b_{i} \widehat{\mathbf{w}}_{l, i}^{c} \quad \text { with } \quad b_{i}=\min _{r \in \mathcal{R}_{i}} b_{i, r},
$$

while the cell average $\widehat{\mathbf{w}}_{1, i}^{n}=\widehat{\mathbf{w}}_{1, i}^{c}$ is ensured by construction thanks to the constrained least square approach used to solve the linear system (67). Once the limiting procedure (69) has been carried out, the final degrees of freedom $\widehat{\mathbf{w}}_{l, i}^{n}$ are known and the limited reconstruction polynomial (65) is fully defined for each cell. The coefficient $b_{i}$ in (69) depends on the element node and is computed according to [4] as

$$
b_{i, r}=\left\{\begin{array}{llc}
\min \left(1, \frac{\mathbf{Q}_{i}^{n, \max }-\mathbf{Q}_{i}^{n}}{\mathbf{w}_{i}^{n}\left(\mathbf{x}_{r}^{n}\right)-\mathbf{Q}_{i}^{n}}\right) & \text { if } & \mathbf{w}_{i}^{n}\left(\mathbf{x}_{r}^{n}\right)>\mathbf{Q}_{i}^{n} \\
\min \left(1, \frac{\mathbf{Q}_{i}^{n, \min }-\mathbf{Q}_{i}^{n}}{\mathbf{w}_{i}^{n}\left(\mathbf{x}_{r}^{n}\right)-\mathbf{Q}_{i}^{n}}\right) & \text { if } & \mathbf{w}_{i}^{n}\left(\mathbf{x}_{r}^{n}\right)<\mathbf{Q}_{i}^{n} \\
1 & \text { if } & \mathbf{w}_{i}^{n}\left(\mathbf{x}_{r}^{n}\right)=\mathbf{Q}_{i}^{n}
\end{array} .\right.
$$

Implicit-Explicit Runge-Kutta time stepping. The second order in time extension is based on an implicit-explicit (IMEX) Runge-Kutta approach [2, 89], that is particularly suitable for discretizing hyperbolic systems of PDE with relaxation source terms. For the sake of clarity and compact notation in the description of the second order in time method, we call $\mathcal{L}_{i m}$ the implicit operator and $\mathcal{L}_{e x}$ the explicit one, hence obtaining the semi-discrete form of the governing equations (2):

$$
\frac{\mathrm{d} \mathbf{Q}}{\mathrm{d} t}=\mathcal{L}_{e x}(t, \mathbf{Q}, \nabla \mathbf{Q})+\mathcal{L}_{i m}(t, \mathbf{Q})
$$

with

$$
\mathcal{L}_{e x}(t, \mathbf{Q}, \nabla \mathbf{Q})=\left[\begin{array}{c}
\rho^{-1} \nabla \cdot \mathbf{v} \\
\rho^{-1} \nabla \cdot \mathbf{T} \\
\rho^{-1} \nabla \cdot(\mathbf{T} \mathbf{v}+\mathbf{q}) \\
\rho^{-1} \nabla \cdot T \boldsymbol{I} \\
-\left(\mathbf{G}_{e} \nabla \mathbf{v}+\nabla \mathbf{v}^{\top} \mathbf{G}_{e}\right)
\end{array}\right], \quad \mathcal{L}_{i m}(t, \mathbf{Q})=\left[\begin{array}{c}
0 \\
0 \\
0 \\
-\mathbf{H} / \Psi \\
2 \boldsymbol{\sigma} /(\rho \Theta)
\end{array}\right]
$$


The vertex-based fluxes are considered explicitly as well as the flux in the trajectory equation (29), because the nonlinear nodal solver described in Section 3.4 reduces to a fully explicit scheme in the stiff relaxation limits of the model (2). In this way, the first order finite volume scheme (35) is compactly written in semi-discrete form as

$$
\mathbf{Q}^{n+1}=\mathbf{Q}^{n}-\Delta t \mathcal{L}_{e x}\left(t^{n}, \mathbf{Q}^{n}, \nabla \mathbf{Q}^{n}\right)+\Delta t \mathcal{L}_{i m}\left(t^{n+1}, \mathbf{Q}^{n+1}\right)
$$

To improve the time accuracy of the first order method (73), we use the second-order ARS(2,2,2) IMEX scheme [2] detailed in Table 1 by means of its two tableaux, where $\beta=1-\sqrt{2} / 2$ and $\alpha=1-1 /(2 \beta)$. Remarking that $\alpha=\beta-1$

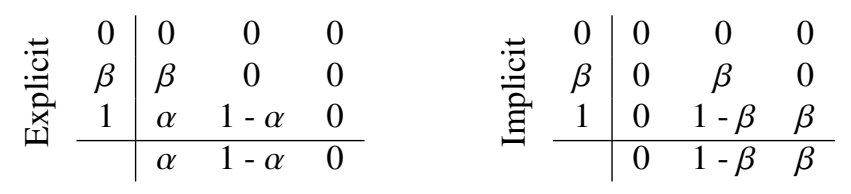

Table 1: Butcher tableaux for the $\operatorname{ARS}(2,2,2)$ time discretization. Left panel: explicit tableau. Right panel: implicit tableau. $\beta=1-\frac{\sqrt{2}}{2}, \alpha=\beta-1$.

and $1-\alpha=2-\beta$, the following two-step scheme for the Lagrangian GPR model (2) is obtained:

$$
\begin{aligned}
\frac{\mathbf{Q}^{(1)}-\mathbf{Q}^{n}}{\Delta t} & =\beta \mathcal{L}_{e x}\left(t^{n}, \mathbf{Q}^{n}, \nabla \mathbf{Q}^{n}\right)+\beta \mathcal{L}_{i m}\left(t^{(1)}, \mathbf{Q}^{(1)}\right), \\
\frac{\mathbf{Q}^{n+1}-\mathbf{Q}^{n}}{\Delta t} & =(\beta-1) \mathcal{L}_{e x}\left(t^{n}, \mathbf{Q}^{n}, \nabla \mathbf{Q}^{n}\right)+(2-\beta) \mathcal{L}_{e x}\left(t^{(1)}, \mathbf{Q}^{(1)}, \nabla \mathbf{Q}^{(1)}\right) \\
& +\beta \mathcal{L}_{i m}\left(\mathbf{Q}^{(1)}\right)+(1-\beta) \mathcal{L}_{i m}\left(t^{(1)}, \mathbf{Q}^{(1)}\right)+\beta \mathcal{L}_{i m}\left(t^{n+1}, \mathbf{Q}^{n+1}\right),
\end{aligned}
$$

where the superscript (1) denotes the intermediate Runge-Kutta stage. The trajectory ODE also follows the explicit tableau, since it contains only vertex-based fluxes, thus one gets

$$
\begin{aligned}
\frac{\mathbf{x}^{(1)}-\mathbf{x}^{n}}{\Delta t} & =\beta \tilde{\mathbf{v}}^{*, n} \\
\frac{\mathbf{x}^{n+1}-\mathbf{x}^{n}}{\Delta t} & =(\beta-1) \tilde{\mathbf{v}}^{*, n}+(2-\beta) \mathbf{v}^{*,(1)} .
\end{aligned}
$$

Let us notice that the $\operatorname{ARS}(2,2,2)$ IMEX scheme in Table 1 has the property of being stiffly accurate (SA), meaning that the last Runge-Kutta stage coincides with the new solution at time $t^{n+1}$. This is a crucial feature for developing asymptotic preserving time discretizations, as detailed in [89]. Furthermore, the scheme is only diagonally implicit, therefore it requires the solution of one implicit equation for the heat source and for the source of the metric tensor equation. This is also confirmed by looking at the time stepping scheme (74)-(75), where the implicit contribution is only taken into account by the terms $\beta \mathcal{L}_{i m}\left(\mathbf{Q}^{(1)}\right)$ in the first stage (74) and $\beta \mathcal{L}_{i m}\left(\mathbf{Q}^{n+1}\right)$ in the second stage (75).

\section{Asymptotic analysis of the scheme}

In this section we analyze the stiff relaxation limits of the fully discrete first order finite volume scheme (35), which are retrieved for $\tau_{1} \rightarrow 0$ and $\tau_{2} \rightarrow 0$. The numerical method is proven to be asymptotic preserving for the viscous stress tensor $\boldsymbol{\sigma}$ and for the thermal impulse $\mathbf{J}$. To that aim, let us introduce the $k$-th order Chapman-Enskog expansion of a generic variable $\phi$ in powers of the stiffness parameter $\tau$ (i.e. $\tau=\tau_{1}$ or $\tau=\tau_{2}$ ), that reads

$$
\phi=\phi_{(0)}+\tau \phi_{(1)}+\tau^{2} \phi_{(2)}+\ldots+O\left(\tau^{k}\right) .
$$


Application of the expansion (78) up to the first order in $\tau$ to $\mathbf{G}_{e_{i}}$ and $\mathbf{J}_{i}$ in the cell-centered evolution equations (35d) and (35e) yields

$$
\begin{aligned}
& \frac{\boldsymbol{G}_{e_{i}(0)}^{n+1}+\tau_{1} \boldsymbol{G}_{e_{i}(1)}^{n+1}-\boldsymbol{G}_{e_{i}(0)}^{n}-\tau_{1} \boldsymbol{G}_{e_{i}(1)}^{n}}{\Delta t}=-\left(\boldsymbol{G}_{e_{i}(0)}^{n}+\tau_{1} \boldsymbol{G}_{e_{i}(1)}^{n}\right) \mathbb{L}_{i}\left(\tilde{\mathbf{v}}^{*}\right)-\mathbb{L}_{i}\left(\tilde{\mathbf{v}}^{*}\right)^{\top}\left(\boldsymbol{G}_{e_{i}(0)}^{n}+\tau_{1} \boldsymbol{G}_{e_{i}(1)}^{n}\right) \\
& +\frac{6}{\tau_{1}}\left|\boldsymbol{G}_{e_{i}(0)}^{n+1}+\tau_{1} \boldsymbol{G}_{e_{i}(1)}^{n+1}\right|^{5 / 6}\left(\boldsymbol{G}_{e_{i}(0)}^{n+1}+\tau_{1} \boldsymbol{G}_{e_{i}(1)}^{n+1}\right)\left({\stackrel{\mathcal{G}}{e_{i}(0)}}^{n+1}+\tau_{1}{\stackrel{\mathbf{G}}{e_{i}(1)}}^{n+1}\right) \\
& +O\left(\tau_{1}^{2}\right) \\
& \frac{\mathbf{J}_{i(0)}^{n+1}+\tau_{2} \mathbf{J}_{i(1)}^{n+1}-\mathbf{J}_{i(0)}^{n}-\tau_{2} \mathbf{J}_{i(1)}^{n}}{\Delta t}=-\frac{1}{m_{i}} \sum_{f \in \mathcal{F}_{i}} \widehat{T_{f i} \boldsymbol{I} \cdot \mathbf{n}_{f i}}{ }^{n} s_{f}^{n}-\frac{1}{\tau_{2}} \frac{T_{i}^{n}}{T_{0}} \frac{\rho_{0}}{\rho_{i}^{n+1}}\left(\mathbf{J}_{i(0)}^{n+1}+\tau_{2} \mathbf{J}_{i(1)}^{n+1}\right)+O\left(\tau_{2}^{2}\right) \text {. }
\end{aligned}
$$

Asymptotic limit of the viscous stress tensor at zeroth order approximation. Multiplying by $\tau_{1}$ equation (79), letting $\tau_{1} \rightarrow 0$ and retaining leading order terms for $\boldsymbol{G}_{e_{i}}$ leads to

$$
6\left|\mathbf{G}_{e_{i}(0)}^{n+1}\right|^{5 / 6} \mathbf{G}_{e_{i}(0)}^{n+1} \stackrel{\circ}{\mathbf{G}}_{e_{i}(0)}^{n+1}=O\left(\tau_{1}^{2}\right) .
$$

According to the GPR model at the continuous level [93] and due to the compatible discretization of the GCL (35a) along the lines of [23], the finite volume scheme (35) ensures the following compatibility condition

$$
\sqrt{\left|\mathbf{G}_{e_{i}}^{n+1}\right|}=\frac{\rho_{i}^{n+1}}{\rho_{0}}+O(\Delta t)>0 .
$$

Therefore, $\left|\boldsymbol{G}_{e_{i}(0)}^{n+1}\right|^{5 / 6}>0$ in (81) and at zeroth leading order the discrete metric tensor becomes trace-free for $\tau_{1} \rightarrow 0$, i.e. ${\stackrel{G}{e_{i}(0)}}_{n+1}^{n+1} O\left(\tau_{1}^{2}\right)$. Inserting this result in the discrete viscous stress tensor (44), we obtain

$$
\boldsymbol{\sigma}_{i}^{n+1}=-\rho_{i}^{n+1} c_{\mathrm{sh}}^{2} \mathbf{G}_{e_{i}(0)}^{n+1} \stackrel{\circ}{\mathbf{G}}_{e_{i}(0)}^{n+1}=O\left(\tau_{1}^{2}\right) .
$$

Hence, viscous stresses vanish and we retrieve the inviscid case which corresponds to the compressible Euler equations. Let us notice that, in the absence of heat conduction $(\alpha=\kappa=0)$, the finite volume scheme (35a)-(35c) exactly reduces to the EUCCLHYD scheme presented in [79]. Furthermore, we can write

$$
\mathbf{G}_{e_{i}(0)}^{n+1}=\frac{1}{3} \operatorname{tr} \mathbf{G}_{e_{i}(0)}^{n+1} \boldsymbol{I}+O\left(\tau_{1}^{2}\right):=g_{i}^{n+1} \boldsymbol{I},
$$

thus allowing us to write the Chapman-Enskog expansion of $\mathbf{G}_{e_{i}}^{n+1}$ as

$$
\mathbf{G}_{e_{i}}^{n+1}=g_{i}^{n+1} \boldsymbol{I}+\tau_{1} \mathbf{G}_{e_{i}(1)}^{n+1}+O\left(\tau_{1}^{2}\right)
$$

The unknown coefficient $g_{i}^{n+1}$ can be determined by computing the determinant of $\mathbf{G}_{e_{i}}^{n+1}$ from the above relation and neglecting terms of the order $O\left(\tau_{1}\right)$, that is

$$
\left|\mathbf{G}_{e_{i}}^{n+1}\right|=\left(g_{i}^{n+1}\right)^{3}+O\left(\tau_{1}\right), \quad \Rightarrow \quad g_{i}^{n+1}=\left(\frac{\rho_{i}^{n+1}}{\rho_{0}}\right)^{2 / 3}+O\left(\tau_{1}\right) .
$$

Asymptotic limit of the viscous stress tensor at first order approximation. Here, a first order approximation of the viscous stress tensor is analyzed in the stiff limit $\tau_{1} \rightarrow 0$. Let us start from the previous expansion (85), which also implies ${\stackrel{\circ}{e_{i}}}^{n+1}=\tau_{1} \stackrel{\circ}{\mathbf{G}}_{e_{i}(1)}^{n+1}+O\left(\tau_{1}^{2}\right)$. The compatibility condition (82) allows the density $\rho_{i}^{n+1}$ to be computed as

$$
\rho_{i}^{n+1}=\rho_{0}\left|g_{i}^{n+1}+\tau_{1} \mathbf{G}_{e_{i}(1)}^{n+1}\right|^{1 / 2}+O\left(\tau_{1}^{2}\right)=\rho_{0}\left(\left(g_{i}^{n+1}\right)^{3 / 2}+\frac{\tau_{1}}{2}\left(g_{i}^{n+1}\right)^{1 / 2} \operatorname{tr} \mathbf{G}_{e_{i}(1)}^{n+1}\right)+O\left(\tau_{1}^{2}\right),
$$

thus a first order expansion of the discrete viscous stress tensor (44) writes

$$
\boldsymbol{\sigma}_{i}^{n+1}=-\rho_{0} c_{\mathrm{sh}}^{2}\left(\left(g_{i}^{n+1}\right)^{3 / 2}+\frac{\tau_{1}}{2}\left(g_{i}^{n+1}\right)^{1 / 2} \operatorname{tr} \mathbf{G}_{e_{i}(1)}^{n+1}\right)\left(g_{i}^{n+1} \boldsymbol{I}+\tau_{1} \mathbf{G}_{e_{i}(1)}^{n+1}\right) \tau_{1}{\stackrel{\circ}{\mathbf{G}_{i}(1)}}^{n+1}+O\left(\tau_{1}^{4}\right)
$$


By neglecting high order terms in $\tau_{1}$, the above expression leads to

$$
\boldsymbol{\sigma}_{i}^{n+1}=-\tau_{1} \rho_{0} c_{\mathrm{sh}}^{2}\left(g_{i}^{n+1}\right)^{5 / 2} \stackrel{\circ}{G}_{e_{i}(1)}^{n+1}+O\left(\tau_{1}^{2}\right) .
$$

Following [47] and recalling that ${\stackrel{\mathrm{G}}{e_{i}(0)}}^{n+1}=O\left(\tau_{1}^{2}\right)$ from the zeroth approximation asymptotic analysis, the deviatoric operator is applied to the discrete equation (35e), then only first order terms for $\tau_{1}$ are retained, hence obtaining

$$
\mathbf{G}_{e_{i}(0)}^{n+1} \mathbb{L}_{i}\left(\tilde{\mathbf{v}}^{*}\right)+\mathbb{L}_{i}\left(\tilde{\mathbf{v}}^{*}\right)^{\top} \mathbf{G}_{e_{i}(0)}^{n+1}-\frac{2}{3} \operatorname{tr}\left(\boldsymbol{G}_{e_{i}(0)}^{n+1} \mathbb{L}_{i}\left(\tilde{\mathbf{v}}^{*}\right)\right) \boldsymbol{I}=-6\left|\mathbf{G}_{e_{i}(0)}^{n+1}\right|^{7 / 6}{\stackrel{\circ}{\mathbf{G}_{i}(1)}}_{e^{\prime}}^{n+1}+O\left(\tau_{1}^{2}\right)
$$

Using (84), the above equation simplifies to

$$
\begin{aligned}
& g_{i}^{n+1}\left(\mathbb{L}_{i}\left(\tilde{\mathbf{v}}^{*}\right)+\mathbb{L}_{i}\left(\tilde{\mathbf{v}}^{*}\right)^{\top}-\frac{2}{3} \operatorname{tr}\left(\mathbb{L}_{i}\left(\tilde{\mathbf{v}}^{*}\right)\right) \boldsymbol{I}\right)=-6\left(g_{i}^{n+1}\right)^{7 / 2}{\stackrel{\mathbf{G}}{e_{i}(1)}}_{n+1}+O\left(\tau_{1}^{2}\right), \\
& \left(g_{i}^{n+1}\right)^{5 / 2} \stackrel{\circ}{G}_{e_{i}(1)}^{n+1}=-\frac{1}{6}\left(\mathbb{L}_{i}\left(\tilde{\mathbf{v}}^{*}\right)+\mathbb{L}_{i}\left(\tilde{\mathbf{v}}^{*}\right)^{\top}-\frac{2}{3} \operatorname{tr}\left(\mathbb{L}_{i}\left(\tilde{\mathbf{v}}^{*}\right)\right) \boldsymbol{I}\right)+O\left(\tau_{1}^{2}\right) .
\end{aligned}
$$

By substituting now the left hand-side of the above expression into the first order approximation of the viscous stress tensor (89), we eventually obtain

$$
\boldsymbol{\sigma}_{i}^{n+1}=\frac{\tau_{1}}{6} \rho_{0} c_{\mathrm{sh}}^{2}\left(\mathbb{L}_{i}\left(\tilde{\mathbf{v}}^{*}\right)+\mathbb{L}_{i}\left(\tilde{\mathbf{v}}^{*}\right)^{\top}-\frac{2}{3} \operatorname{tr}\left(\mathbb{L}_{i}\left(\tilde{\mathbf{v}}^{*}\right)\right) \boldsymbol{I}\right)+O\left(\tau_{1}^{2}\right),
$$

which is the discrete version of the stress tensor of the compressible Navier-Stokes equations based on Stokes hypothesis, in which the effective dynamic viscosity coefficient is defined by

$$
\mu=\frac{1}{6} \rho_{0} \tau_{1} c_{\mathrm{sh}}^{2}
$$

that retrieves the analytical formulation (11).

Asymptotic limit of the heat flux at first order approximation. From the leading order term $\tau_{2}^{-1}$ in (80), it follows that

$$
\mathbf{J}_{i(0)}^{n+1}=O\left(\tau_{2}^{2}\right) .
$$

Assuming well-prepared initial data, i.e $\mathbf{J}_{i(0)}^{n}=O\left(\tau_{2}^{2}\right)$, and inserting the above result into (80) while letting $\tau_{2} \rightarrow 0$ yields

$$
\mathbf{J}_{i(1)}^{n+1}=-\frac{T_{0}}{T_{i}^{n}} \frac{\rho_{i}^{n+1}}{\rho_{0}} \frac{1}{m_{i}} \sum_{f \in \mathcal{F}_{i}}{\widehat{T_{f i} \boldsymbol{I} \cdot \mathbf{n}_{f i}}}^{n} s_{f}^{n}+\mathcal{O}\left(\tau_{2}^{2}\right) .
$$

Finally, using (94) and (95) in the Chapman-Enskog expansion (78), we obtain

$$
\mathbf{J}_{i}^{n+1}=-\tau_{2} \frac{T_{0}}{T_{i}^{n}} \frac{1}{\rho_{0}} \frac{1}{\left|T_{i}^{n+1}\right|} \sum_{f \in \mathcal{F}_{i}}{\widehat{T_{f i} \boldsymbol{I} \cdot \mathbf{n}_{f i}}}^{n} s_{f}^{n}+\mathcal{O}\left(\tau_{2}^{2}\right), \quad\left|T_{i}^{n+1}\right|=m_{i} / \rho_{i}^{n+1} .
$$

Therefore, in the stiff relaxation limit, the discrete heat flux vector $\mathbf{q}_{i}^{n}=\alpha^{2} T_{i}^{n} \mathbf{J}_{i}^{n}$ in the energy equation (35c) becomes

$$
\mathbf{q}_{i}^{n}=-\tau_{2} \alpha^{2} \frac{T_{0}}{\rho_{0}} \frac{1}{\left|T_{i}^{n}\right|} \sum_{f \in \mathcal{F}_{i}}{\widehat{T_{f i} \boldsymbol{I} \cdot \mathbf{n}_{f i}}}^{n} s_{f}^{n}+\mathcal{O}\left(\tau_{2}^{2}\right)
$$

Notice that the term $1 /\left|T_{i}^{n}\right| \sum_{f \in \mathcal{F}_{i}}{\widehat{T_{f i} \boldsymbol{I} \cdot \mathbf{n}_{f i}}}_{f}^{n} s_{f}^{n}$ in (97) is nothing but the discrete gradient operator for the temperature, i.e. $\nabla T$. Hence, taking the heat conduction coefficient $\kappa$ as in (16), equation (97) is an asymptotically consistent discretization of the Fourier law of heat conduction $\mathbf{q}=-\kappa \nabla T$, that is recovered by the finite volume scheme (35) in the stiff relaxation limit $\tau_{2} \rightarrow 0$. 


\section{Numerical results}

In this section, we perform some numerical applications which aim at demonstrating the accuracy and the robustness of the new Lagrangian finite volume scheme (35) for the solution of the GPR model (2). We refer to this numerical algorithm with the abbreviation LGPR. A wide range of test cases is proposed, that covers simulations for ideal and viscous heat conducting fluids, elasto-plastic solids as well as purely elastic solids. We emphasize that for each test case the full GPR model is solved, without neglecting any evolution equation.

The setup of the test problems shown hereafter requires some parameters that will be specified, the most important being the relaxation times $\tau_{1}$ and $\tau_{2}$ which determine the mechanical amd thermodynamical behavior of the material under consideration. The parameters of the GPR model are summarized in Table 2, together with the equation of state used for each test problem. For solids, the shear modulus is always computed as $G=\rho_{0} c_{\mathrm{sh}}^{2}$, while for fluids either the viscosity $\mu$ or the relaxation time $\tau_{1}$ must be prescribed, which are linked by the relation (11). In the case of heat conducting fluids, the relaxation time $\tau_{2}$ is deduced from (16). The adiabatic sound speed $c_{0}$ for ideal gas EOS is evaluated according to (5), for Mie-Grüneisen EOS it is a parameter of the material, whereas for Neo-Hookean materials relation (9) holds true.

Table 2: Parameters of the GPR model for the test problems shown in Section 5. The hydrodynamics equation of state (EOS) is chosen among the ideal gas (IG), Mie-Grüneisen (MG) or Neo-Hookean (NH).

\begin{tabular}{l|ccccccccc|c} 
Test & $\rho_{0}$ & $c_{\mathrm{sh}}$ & $\mu$ & $c_{v}$ & $\alpha$ & $\kappa$ & $T_{0}$ & $\tau_{1}$ & $\tau_{2}$ & EOS \\
\hline Isentropic vortex & 1 & 0.5 & - & 1 & 0 & 0 & 1 & $10^{-14}$ & $10^{-14}$ & IG \\
Swinging plate & 1100 & 73 & - & 1 & 0 & 0 & 1 & $10^{14}$ & $10^{-14}$ & $\mathrm{NH}$ \\
Kidder & 1 & 0.5 & - & 1 & 0 & 0 & 1 & $10^{-14}$ & $10^{-14}$ & IG \\
Saltzman & 1 & 0.5 & - & 1 & 0 & 0 & 1 & $10^{-14}$ & $10^{-14}$ & IG \\
Sedov & 1 & 0.5 & - & 1 & 0 & 0 & 1 & $10^{-14}$ & $10^{-14}$ & IG \\
Riemann problems & 1 & 10 & {$\left[10^{-3} ; 10^{-2}\right]$} & 2.5 & 0 & 0 & 1 & $(11)$ & $10^{-14}$ & IG \\
Heat conduction & 1 & 1 & $10^{-2}$ & 2.5 & 2 & $10^{-2}$ & 1 & $(11)$ & $(16)$ & IG \\
Viscous shock & 1 & 50 & $2 \cdot 10^{-2}$ & 2.5 & 50 & $9.33 \cdot 10^{-2}$ & 1 & $(11)$ & $(16)$ & IG \\
Shell collapse & 1845 & 9073.62 & - & 1 & 0 & 0 & 1 & $(12)$ & $10^{-14}$ & MG \\
2D projectile & 2785 & 3150 & - & 1 & 0 & 0 & 1 & $(12)$ & $10^{-14}$ & MG \\
3D Taylor bar & 8930 & 2245 & - & 1 & 0 & 0 & 1 & $(12)$ & $10^{-14}$ & MG \\
Elastic plate & 1845 & 9046.59 & - & 1 & 0 & 0 & 1 & $10^{14}$ & $10^{-14}$ & $\mathrm{NH}$ \\
Twisting column & 1100 & 73 & - & 1 & 0 & 0 & 1 & $10^{14}$ & $10^{-14}$ & $\mathrm{NH}$
\end{tabular}

If not specified, the material is initially unloaded, thus we set $\boldsymbol{G}_{e}=\left(\rho / \rho_{0}\right)^{2 / 3} \boldsymbol{I}$ according to (86). Furthermore, the thermal impulse vector is initialized with $\mathbf{J}=\mathbf{0}$ and the initial density distribution is $\rho=\rho_{0}$. The CFL number in (18) is taken to be CFL $=0.45$ in $2 \mathrm{D}$ and CFL $=0.3$ in $3 \mathrm{D}$. The simulations are run using the fully second order space-time scheme described in Section 3.6.

Physical units are based on the $[m, k g, s]$ unit system, thus Young and shear moduli for solids are measured in $[\mathrm{Pa}]$, as well as pressure and stresses. Energy is expressed in Joule $[\mathrm{J}]$.

\subsection{Numerical convergence studies}

The convergence studies of the LGPR scheme are carried out by considering both limits of the model, namely for $\tau_{1} \rightarrow 0$ and $\tau_{1} \rightarrow \infty$. The first case corresponds to the simulation of an ideal inviscid fluid (see Section 4 ), while the latter is concerned with a purely elastic material. These particular limits of the model permit to retrieve at the discrete level the EUCCLHYD scheme [79] for hydrodynamics, and the cell-centered Lagrangian algorithm for nearly incompressible hyperelastic solids presented in [23]. 
Convergence studies in the stiff limit $\tau_{1} \rightarrow 0$. We consider the isentropic vortex problem initially introduced in a two-dimensional setting [64] for the compressible Euler equations. The initial computational domain is $\Omega^{2 D}(0)=$ $[0 ; 10] \times[0 ; 10]$ and $\Omega^{3 D}(0)=[0 ; 10] \times[0 ; 10] \times[0 ; 5]$ with periodic boundaries. The ideal gas EOS (5) is used with $\gamma=1.4$, and the relaxation time is set to $\tau_{1}=10^{-14}$, so that the hydrodynamic limit is retrieved. The fluid is characterized by a homogeneous background field on the top of which some perturbations are added, thus

$$
\rho=\rho_{0}+\delta \rho, \quad u=u_{c}+\delta u, \quad v=v_{c}+\delta v, \quad w=w_{c}, \quad T=T_{0}+\delta T,
$$

where the perturbations for density and pressure read

$$
\delta \rho=(1+\delta T)^{\frac{1}{\gamma-1}}-1, \quad \delta p=(1+\delta T)^{\frac{\gamma}{\gamma-1}}-1,
$$

with the temperature fluctuation $\delta T=-\frac{(\gamma-1) \lambda^{2}}{8 \gamma \pi^{2}} e^{1-r^{2}}$. According to [64], the vortex strength is $\lambda=5$ and it moves with a convective velocity $\mathbf{v}_{c}=\left(u_{c}, v_{c}, w_{c}\right)=(1,1,0)$. The velocity field is affected by the following perturbations:

$$
\left(\begin{array}{l}
\delta u \\
\delta v
\end{array}\right)=\frac{\lambda}{2 \pi} e^{\frac{1-r^{2}}{2}}\left(\begin{array}{r}
-y^{\prime} \\
x^{\prime}
\end{array}\right), \quad x^{\prime}=x-x_{c}, \quad y^{\prime}=y-y_{c}
$$

where the center of the vortex is $\left(x_{c}, y_{c}, z_{c}\right)=(5,5,2.5)$ and the generic radial position is $r=\sqrt{x^{\prime 2}+y^{\prime 2}}$. The final time of the simulation is chosen to be $t_{f}=0.1$ and the exact solution is simply given by the time-advected initial condition with convective velocity $\mathbf{v}_{c}$. The simulation is performed on a sequence of four successively refined unstructured grids in $2 \mathrm{D}$ and in $3 \mathrm{D}$, and the numerical convergence results are reported in Table 3. The errors are measured in $L_{2}$ norm for the variables $\{\omega, u, E\}$, and $h\left(\Omega\left(t_{f}\right)\right)$ represents the mesh size which is taken to be the maximum diameter of the circumspheres or the circumcircles of the elements in the final domain configuration $\Omega\left(t_{f}\right)$. The expected first and second order of accuracy are achieved in all cases.

Convergence studies in the limit $\tau_{1} \rightarrow \infty$. Here, we study the convergence of the LGPR scheme when $\tau_{1}=10^{14}$, thus the source term in the metric tensor equation vanishes and purely elastic solids are simulated. The swinging plate/cube test problem is considered, according to the setup proposed in [102, 103]. The initial computational domain is $\Omega(0)=[0 ; 2]^{d}$ and the analytical solution for the velocity is given by

$$
\begin{gathered}
\mathbf{v}^{2 D}(t, \mathbf{x})=\Lambda U_{0} \cos (\Lambda t)\left(\begin{array}{r}
-\sin \left(\frac{\pi}{2} x\right) \cos \left(\frac{\pi}{2} y\right) \\
\cos \left(\frac{\pi}{2} x\right) \sin \left(\frac{\pi}{2} y\right)
\end{array}\right), \quad \Lambda=\frac{\pi}{2} \sqrt{\frac{2 G}{\rho_{0}}} \\
\mathbf{v}^{3 D}(t, \mathbf{x})=\Lambda U_{0} \cos (\Lambda t)\left(\begin{array}{r}
-2 \sin \left(\frac{\pi}{2} x\right) \cos \left(\frac{\pi}{2} y\right) \cos \left(\frac{\pi}{2} z\right) \\
\cos \left(\frac{\pi}{2} x\right) \sin \left(\frac{\pi}{2} y\right) \cos \left(\frac{\pi}{2} z\right) \\
\cos \left(\frac{\pi}{2} x\right) \cos \left(\frac{\pi}{2} y\right) \sin \left(\frac{\pi}{2} z\right)
\end{array}\right), \quad \Lambda=\pi \sqrt{\frac{3 G}{4 \rho_{0}}}
\end{gathered}
$$

with $U_{0}=5 \cdot 10^{-4}$. The Neo-Hookean hydrodynamic EOS (7) and shear elastic energy ( $3 \mathrm{~b}$ ) are adopted, with Poisson ratio $v=0.45$ and Young modulus $Y=1.7 \cdot 10^{7}$, thus a nearly incompressible solid is modeled. The velocity and displacement fields are divergence-free, leading to the exact pressure $p=0$. Space-time dependent boundary conditions are prescribed for the normal velocities, according to the exact solution (102). The final time of the simulation is chosen to be $t_{f}=\pi / \Lambda$, thus the final displacement corresponds to the initial one. As done for the stiff limit case, the swinging plate/cube test is run on a sequence of triangular and tetrahedral meshes that become finer, hence allowing the convergence rates to be computed. The results are collected in Table 4, demonstrating that the formal order of accuracy is achieved even when simulating elastic solids with the same set of equations and the same LGPR scheme (35) used for the hydrodynamics limit.

\subsection{Kidder problem}

The Kidder test case [71] describes the isentropic compression of a shell filled with perfect gas that is initially bounded between the internal radius $r_{i n t}=0.9$ and the external radius $r_{\text {ext }}=1.0$. The initial condition is given in terms 
Table 3: Numerical convergence results for the isentropic vortex test in $2 \mathrm{D}$ and in $3 \mathrm{D}$ using the LGPR scheme with relaxation time $\tau_{1}=10^{-14}$ on a sequence of refined unstructured meshes of size $h\left(\Omega\left(t_{f}\right)\right)$ measured at the end of the simulation $t_{f}=0.1$. The errors are measured in $L_{2}$ norm for specific volume $\omega$, horizontal velocity $u$ and total energy $E$.

\begin{tabular}{c|cccccc}
\multicolumn{7}{c}{ 2D LGPR $O 1\left(\tau_{1}=10^{-14}\right)$} \\
\hline$h\left(\Omega\left(t_{f}\right)\right)$ & $(\omega)_{L_{2}}$ & $O(1 / \rho)$ & $u_{L_{2}}$ & $O(u)$ & $E_{L_{2}}$ & $O(E)$ \\
\hline $3.26 \mathrm{E}-01$ & $5.405 \mathrm{E}-02$ & - & $1.547 \mathrm{E}-01$ & - & $2.579 \mathrm{E}-01$ & - \\
$2.47 \mathrm{E}-01$ & $4.164 \mathrm{E}-02$ & 0.96 & $1.219 \mathrm{E}-01$ & 0.88 & $2.044 \mathrm{E}-01$ & 0.86 \\
$1.63 \mathrm{E}-01$ & $3.053 \mathrm{E}-02$ & 0.74 & $8.866 \mathrm{E}-02$ & 0.76 & $1.471 \mathrm{E}-01$ & 0.78 \\
$1.28 \mathrm{E}-01$ & $2.286 \mathrm{E}-02$ & 1.20 & $7.041 \mathrm{E}-02$ & 0.96 & $1.164 \mathrm{E}-01$ & 0.97
\end{tabular}

\begin{tabular}{c|cccccc}
\multicolumn{7}{c}{ 2D LGPR $O 2\left(\tau_{1}=10^{-14}\right)$} \\
\hline$h\left(\Omega\left(t_{f}\right)\right)$ & $(\omega)_{L_{2}}$ & $O(1 / \rho)$ & $u_{L_{2}}$ & $O(u)$ & $E_{L_{2}}$ & $O(E)$ \\
\hline $3.26 \mathrm{E}-01$ & $4.996 \mathrm{E}-02$ & - & $4.895 \mathrm{E}-02$ & - & $9.281 \mathrm{E}-02$ & - \\
$2.47 \mathrm{E}-01$ & $3.312 \mathrm{E}-02$ & 1.49 & $3.020 \mathrm{E}-02$ & 1.76 & $5.509 \mathrm{E}-02$ & 1.90 \\
$1.63 \mathrm{E}-01$ & $1.913 \mathrm{E}-02$ & 1.32 & $1.534 \mathrm{E}-02$ & 1.63 & $2.858 \mathrm{E}-02$ & 1.58 \\
$1.28 \mathrm{E}-01$ & $1.327 \mathrm{E}-02$ & 1.51 & $9.153 \mathrm{E}-03$ & 2.13 & $1.770 \mathrm{E}-02$ & 1.98
\end{tabular}

\begin{tabular}{c|cccccc}
\multicolumn{7}{c}{ 3D LGPR $O 1\left(\tau_{1}=10^{-14}\right)$} \\
\hline$h\left(\Omega\left(t_{f}\right)\right)$ & $(\omega)_{L_{2}}$ & $O(1 / \rho)$ & $u_{L_{2}}$ & $O(u)$ & $E_{L_{2}}$ & $O(E)$ \\
\hline $5.29 \mathrm{E}-01$ & $2.389 \mathrm{E}-01$ & - & $5.600 \mathrm{E}-01$ & - & $8.781 \mathrm{E}-01$ & - \\
$3.62 \mathrm{E}-01$ & $2.013 \mathrm{E}-01$ & 0.35 & $4.075 \mathrm{E}-01$ & 0.65 & $6.660 \mathrm{E}-01$ & 0.56 \\
$2.31 \mathrm{E}-01$ & $1.752 \mathrm{E}-01$ & 0.31 & $2.882 \mathrm{E}-01$ & 0.77 & $4.877 \mathrm{E}-01$ & 0.69 \\
$1.81 \mathrm{E}-01$ & $1.454 \mathrm{E}-01$ & 0.76 & $2.301 \mathrm{E}-01$ & 0.91 & $3.974 \mathrm{E}-01$ & 0.83
\end{tabular}

\begin{tabular}{c|cccccc}
\multicolumn{7}{c}{ 3D LGPR $O 2\left(\tau_{1}=10^{-14}\right)$} \\
\hline$h\left(\Omega\left(t_{f}\right)\right)$ & $(\omega)_{L_{2}}$ & $O(1 / \rho)$ & $u_{L_{2}}$ & $O(u)$ & $E_{L_{2}}$ & $O(E)$ \\
\hline $5.29 \mathrm{E}-01$ & $2.899 \mathrm{E}-01$ & - & $2.946 \mathrm{E}-01$ & - & $5.185 \mathrm{E}-01$ & - \\
$3.62 \mathrm{E}-01$ & $1.426 \mathrm{E}-01$ & 1.44 & $1.188 \mathrm{E}-01$ & 1.85 & $2.275 \mathrm{E}-01$ & 1.67 \\
$2.31 \mathrm{E}-01$ & $8.304 \mathrm{E}-02$ & 1.20 & $5.829 \mathrm{E}-02$ & 1.59 & $1.099 \mathrm{E}-01$ & 1.62 \\
$1.81 \mathrm{E}-01$ & $5.931 \mathrm{E}-02$ & 1.37 & $3.600 \mathrm{E}-02$ & 1.96 & $7.206 \mathrm{E}-02$ & 1.72
\end{tabular}


Table 4: Numerical convergence results for the swinging plate test in $2 \mathrm{D}$ and in $3 \mathrm{D}$ using the LGPR scheme with relaxation time $\tau_{1}=10^{14}$ on a sequence of refined unstructured meshes of size $h\left(\Omega\left(t_{f}\right)\right)$ measured at the end of the simulation $t_{f}=\pi / \omega$. The errors are measured in $L_{2}$ norm for horizontal velocity $u$, total energy $E$, metric tensor component $\boldsymbol{G}_{e_{11}}$ and Cauchy stress component $\boldsymbol{T}_{11}$.

\begin{tabular}{c|cccccccc}
\multicolumn{8}{c}{ 2D LGPR $O 1\left(\tau_{1}=10^{14}\right)$} \\
\hline$h\left(\Omega\left(t_{f}\right)\right)$ & $u_{L_{2}}$ & $O(u)$ & $E_{L_{2}}$ & $O(E)$ & $\left(\mathbf{G}_{e_{11}}\right)_{L_{2}}$ & $O\left(\boldsymbol{G}_{e_{11}}\right)$ & $\left(\boldsymbol{T}_{11}\right)_{L_{2}}$ & $O\left(\boldsymbol{T}_{11}\right)$ \\
\hline $1.56 \mathrm{E}-01$ & $6.928 \mathrm{E}-02$ & - & $3.926 \mathrm{E}-03$ & - & $1.815 \mathrm{E}-04$ & - & $1.126 \mathrm{E}+03$ & - \\
$7.78 \mathrm{E}-02$ & $5.291 \mathrm{E}-02$ & 0.39 & $3.100 \mathrm{E}-03$ & 0.34 & $1.093 \mathrm{E}-04$ & 0.73 & $6.707 \mathrm{E}+02$ & 0.74 \\
$5.46 \mathrm{E}-02$ & $4.134 \mathrm{E}-02$ & 0.70 & $2.712 \mathrm{E}-03$ & 0.38 & $6.766 \mathrm{E}-05$ & 1.35 & $4.225 \mathrm{E}+02$ & 1.30 \\
$3.92 \mathrm{E}-02$ & $3.374 \mathrm{E}-02$ & 0.61 & $2.338 \mathrm{E}-03$ & 0.45 & $4.620 \mathrm{E}-05$ & 1.15 & $2.958 \mathrm{E}+02$ & 1.08
\end{tabular}

\begin{tabular}{c|cccccccc}
\multicolumn{8}{c}{ 2D LGPR $O 2\left(\tau_{1}=10^{14}\right)$} \\
\hline$h\left(\Omega\left(t_{f}\right)\right)$ & $u_{L_{2}}$ & $O(u)$ & $E_{L_{2}}$ & $O(E)$ & $\left(\mathbf{G}_{e_{11}}\right)_{L_{2}}$ & $O\left(\boldsymbol{G}_{e_{11}}\right)$ & $\left(\boldsymbol{T}_{11}\right)_{L_{2}}$ & $O\left(\boldsymbol{T}_{11}\right)$ \\
\hline $1.56 \mathrm{E}-01$ & $1.377 \mathrm{E}-02$ & - & $1.505 \mathrm{E}-03$ & - & $1.696 \mathrm{E}-04$ & - & $1.303 \mathrm{E}+03$ & - \\
$7.78 \mathrm{E}-02$ & $2.888 \mathrm{E}-03$ & 2.24 & $2.730 \mathrm{E}-04$ & 2.45 & $4.224 \mathrm{E}-04$ & 1.99 & $2.845 \mathrm{E}+02$ & 2.18 \\
$5.46 \mathrm{E}-02$ & $1.131 \mathrm{E}-03$ & 2.65 & $9.735 \mathrm{E}-05$ & 2.91 & $1.860 \mathrm{E}-05$ & 2.31 & $1.228 \mathrm{E}+02$ & 2.37 \\
$3.92 \mathrm{E}-02$ & $6.081 \mathrm{E}-04$ & 1.87 & $5.080 \mathrm{E}-05$ & 1.96 & $1.088 \mathrm{E}-05$ & 1.62 & $6.990 \mathrm{E}+01$ & 1.70
\end{tabular}

\begin{tabular}{c|cccccccc}
\multicolumn{8}{c}{ 3D LGPR $O 1\left(\tau_{1}=10^{14}\right)$} \\
\hline$h\left(\Omega\left(t_{f}\right)\right)$ & $u_{L_{2}}$ & $O(u)$ & $E_{L_{2}}$ & $O(E)$ & $\left(\boldsymbol{G}_{e_{11}}\right)_{L_{2}}$ & $O\left(\boldsymbol{G}_{e_{11}}\right)$ & $\left(\boldsymbol{T}_{11}\right)_{L_{2}}$ & $O\left(\boldsymbol{T}_{11}\right)$ \\
\hline $1.56 \mathrm{E}-01$ & $6.179 \mathrm{E}-02$ & - & $5.892 \mathrm{E}-03$ & - & $1.859 \mathrm{E}-04$ & - & $5.111 \mathrm{E}+03$ & - \\
$7.78 \mathrm{E}-02$ & $5.190 \mathrm{E}-02$ & 0.77 & $5.286 \mathrm{E}-03$ & 0.48 & $1.639 \mathrm{E}-04$ & 0.56 & $4.676 \mathrm{E}+03$ & 0.39 \\
$5.46 \mathrm{E}-02$ & $4.845 \mathrm{E}-02$ & 0.91 & $4.971 \mathrm{E}-03$ & 0.81 & $1.603 \mathrm{E}-04$ & 0.30 & $4.631 \mathrm{E}+03$ & 0.15 \\
$3.92 \mathrm{E}-02$ & $4.219 \mathrm{E}-02$ & 1.06 & $4.433 \mathrm{E}-03$ & 0.88 & $1.473 \mathrm{E}-04$ & 0.65 & $4.279 \mathrm{E}+03$ & 0.61
\end{tabular}

\begin{tabular}{c|cccccccc}
\multicolumn{8}{c}{ 3D LGPR $O 2\left(\tau_{1}=10^{14}\right)$} \\
\hline$h\left(\Omega\left(t_{f}\right)\right)$ & $u_{L_{2}}$ & $O(u)$ & $E_{L_{2}}$ & $O(E)$ & $\left(\boldsymbol{G}_{e_{11}}\right)_{L_{2}}$ & $O\left(\boldsymbol{G}_{e_{11}}\right)$ & $\left(\boldsymbol{T}_{11}\right)_{L_{2}}$ & $O\left(\boldsymbol{T}_{11}\right)$ \\
\hline $1.56 \mathrm{E}-01$ & $1.792 \mathrm{E}-02$ & - & $2.752 \mathrm{E}-03$ & - & $1.297 \mathrm{E}-04$ & - & $4.262 \mathrm{E}+03$ & - \\
$7.78 \mathrm{E}-02$ & $1.171 \mathrm{E}-02$ & 2.08 & $1.864 \mathrm{E}-03$ & 1.91 & $1.080 \mathrm{E}-04$ & 0.89 & $3.606 \mathrm{E}+03$ & 0.82 \\
$5.46 \mathrm{E}-02$ & $7.487 \mathrm{E}-03$ & 1.69 & $1.223 \mathrm{E}-03$ & 1.59 & $8.105 \mathrm{E}-05$ & 1.09 & $2.723 \mathrm{E}+03$ & 1.06 \\
$3.92 \mathrm{E}-02$ & $6.235 \mathrm{E}-03$ & 2.24 & $1.013 \mathrm{E}-03$ & 2.31 & $7.160 \mathrm{E}-05$ & 1.52 & $2.405 \mathrm{E}+03$ & 1.52
\end{tabular}


of the general radial coordinate $r=\sqrt{\mathbf{x}^{2}}$ and it reads

$$
\left(\begin{array}{c}
\rho(0, r) \\
\mathbf{v}(0, r) \\
p(0, r)
\end{array}\right)=\left(\begin{array}{c}
\left(\frac{r_{e x t}^{2}-r^{2}}{r_{e x t}^{2}-r_{i n t}^{2}} \rho_{i n t}^{\gamma-1}+\frac{r^{2}-r_{i n t}^{2}}{r_{e x t}^{2}-r_{e x t}^{2}} \rho_{e x t}^{\gamma-1}\right)^{-\frac{1}{\gamma-1}} \\
0 \\
s \rho(0, r)^{\gamma}
\end{array}\right)
$$

where $\rho_{\text {int }}=1$ and $\rho_{\text {ext }}=2$ are the initial values of density at the corresponding frontier of the shell, while $\gamma=5 / 3$ is the ratio of specific heats for the ideal gas EOS. The initial entropy distribution is assumed to be uniform, hence $s=\frac{p}{\rho^{\gamma}}=1$. The final time $t_{f}=\frac{\sqrt{3}}{2} \tilde{t}$ is determined in such a way that the shell is bounded by $0.45 \leq r \leq 0.5$, which provides a reference solution for the shell configuration at the end of the simulation. The focalisation time is given by $\tilde{t}=\sqrt{\frac{\gamma-1}{2} \frac{\left(r_{e x t}^{2}-r_{i n t}^{2}\right)}{c_{e x t}^{2}-c_{i n t}^{2}}}$, with the internal and external sound speeds $c_{i n t}=\sqrt{\gamma \frac{p_{\text {int }}}{\rho_{\text {int }}}}$ and $c_{e x t}=\sqrt{\gamma \frac{p_{e x t}}{\rho_{e x t}}}$. Pressure boundary conditions are set on the internal and external frontiers of the shell according to the exact solution available in [71]. Since this is a smooth problem, the numerical convergence is studied again on a sequence of successively refined computational meshes. The errors are measured in $L_{\infty}$ norm and reported in Table 5 for the second order version of the LGPR scheme, where the formal accuracy is reached in $2 \mathrm{D} / 3 \mathrm{D}$. The corresponding errors related to the internal and external frontier location, i.e. $R_{\text {int }}(t)$ and $R_{\text {ext }}(t)$, are estimated as the arithmetic average of the difference between the analytical and the numerical radial coordinate for each node lying on the internal and external frontier.

Table 5: Numerical convergence results for the Kidder problem in $2 \mathrm{D}$ and in $3 \mathrm{D}$ using the LGPR scheme with relaxation time $\tau_{1}=10^{-14}$ on a sequence of refined unstructured triangular and tetrahedral meshes of size $h\left(\Omega\left(t_{f}\right)\right)$ measured at the end of the simulation $t_{f}$. The errors are measured in $L_{\infty}$ norm for specific volume $1 / \rho$, total energy $E$, final position of the internal $R_{\text {int }}$ and external $R_{\text {ext }}$ radius of the shell.

\begin{tabular}{|c|c|c|c|c|c|c|c|c|}
\hline \multicolumn{9}{|c|}{ 2D LGPR $O 2$} \\
\hline$h\left(\Omega\left(t_{f}\right)\right)$ & $(1 / \rho)_{L_{\infty}}$ & $O(1 / \rho)$ & $E_{L_{\infty}}$ & $O(E)$ & $\left(R_{\text {int }}\right)_{L_{\infty}}$ & $O\left(R_{\text {int }}\right)$ & $\left(R_{e x t}\right)_{L_{\infty}}$ & $O\left(R_{e x t}\right)$ \\
\hline $2.15 \mathrm{E}-02$ & $7.648 \mathrm{E}-02$ & - & $5.302 \mathrm{E}+00$ & - & $5.538 \mathrm{E}-03$ & - & $5.001 \mathrm{E}-03$ & - \\
\hline $1.42 \mathrm{E}-02$ & $2.900 \mathrm{E}-02$ & 2.36 & $2.316 \mathrm{E}+00$ & 2.01 & $2.061 \mathrm{E}-03$ & 2.40 & $2.122 \mathrm{E}-03$ & 2.08 \\
\hline $7.95 \mathrm{E}-03$ & $1.356 \mathrm{E}-02$ & 1.30 & $4.888 \mathrm{E}-01$ & 2.67 & 5.991E-04 & 2.12 & 7.702E-04 & 1.74 \\
\hline 4.14E-03 & $3.836 \mathrm{E}-03$ & 1.94 & $1.670 \mathrm{E}-01$ & 1.65 & $2.145 \mathrm{E}-04$ & 1.57 & $2.528 \mathrm{E}-04$ & 1.71 \\
\hline \multicolumn{9}{|c|}{ 3D LGPR $O 2$} \\
\hline$h\left(\Omega\left(t_{f}\right)\right)$ & $(1 / \rho)_{L_{\infty}}$ & $O(1 / \rho)$ & $E_{L_{\infty}}$ & $O(E)$ & $\left(R_{\text {int }}\right)_{L_{\infty}}$ & $O\left(R_{\text {int }}\right)$ & $\left(R_{e x t}\right)_{L_{\infty}}$ & $O\left(R_{e x t}\right)$ \\
\hline $2.15 \mathrm{E}-02$ & $4.150 \mathrm{E}+00$ & - & $2.162 \mathrm{E}+01$ & - & $9.825 \mathrm{E}-02$ & - & $1.810 \mathrm{E}-02$ & - \\
\hline $1.42 \mathrm{E}-02$ & $2.982 \mathrm{E}+00$ & 2.27 & $1.757 \mathrm{E}+01$ & 1.42 & $5.620 \mathrm{E}-02$ & 3.84 & $1.101 \mathrm{E}-02$ & 3.41 \\
\hline 7.95E-03 & $1.327 \mathrm{E}-01$ & 7.22 & $9.911 \mathrm{E}+00$ & 1.33 & 3.009E-02 & 1.45 & $7.078 \mathrm{E}-03$ & 1.03 \\
\hline 4.14E-03 & $5.634 \mathrm{E}-02$ & 1.80 & $3.437 \mathrm{E}+00$ & 2.22 & $1.161 \mathrm{E}-02$ & 2.00 & $3.343 \mathrm{E}-03$ & 1.58 \\
\hline
\end{tabular}

Figure 3 depicts the initial and final pressure distribution in 2D and 3D, which preserves a good symmetry despite the unstructured meshes. The time evolution of the internal and external frontiers of the shell is compared against the analytical solution during the entire simulation, exhibiting an excellent agreement.

\subsection{Saltzman problem}

The Saltzman problem involves a strong shock wave that is generated by the motion of a piston traveling along the main direction of a rectangular box. This is a challenging test case first proposed in $[44,30]$ on a skewed quadrangular mesh to verify the robustness of Lagrangian schemes when the mesh is not aligned with the fluid flow. The initial computational domain is $\Omega^{2 D}(0)=[0 ; 1] \times[0 ; 0.1]$ and $\Omega^{3 D}(0)=[0 ; 1] \times[0 ; 0.1] \times[0 ; 0.1]$, with zero velocity slip-wall boundary conditions everywhere, except for the piston, which is assigned with moving slip-wall boundary condition. The usage of unstructured meshes allows any prescribed skewness to be ignored, since the computational mesh does not exhibit any face aligned with the fluid flow, as depicted in Figure 4 at the final time of the simulation $t_{f}=0.6$. The characteristic mesh size is $h=1 / 100$ for both 2D and 3D grids.

The initial pressure of the fluid is $p=10^{-6}$ and the ideal gas EOS is used with $\gamma=5 / 3$. The piston lies on the left side of the domain and moves with velocity $\mathbf{v}_{p}=(1,0,0)$. The CFL number is set to CFL $=0.01$ up to time $t=0.01$ in 

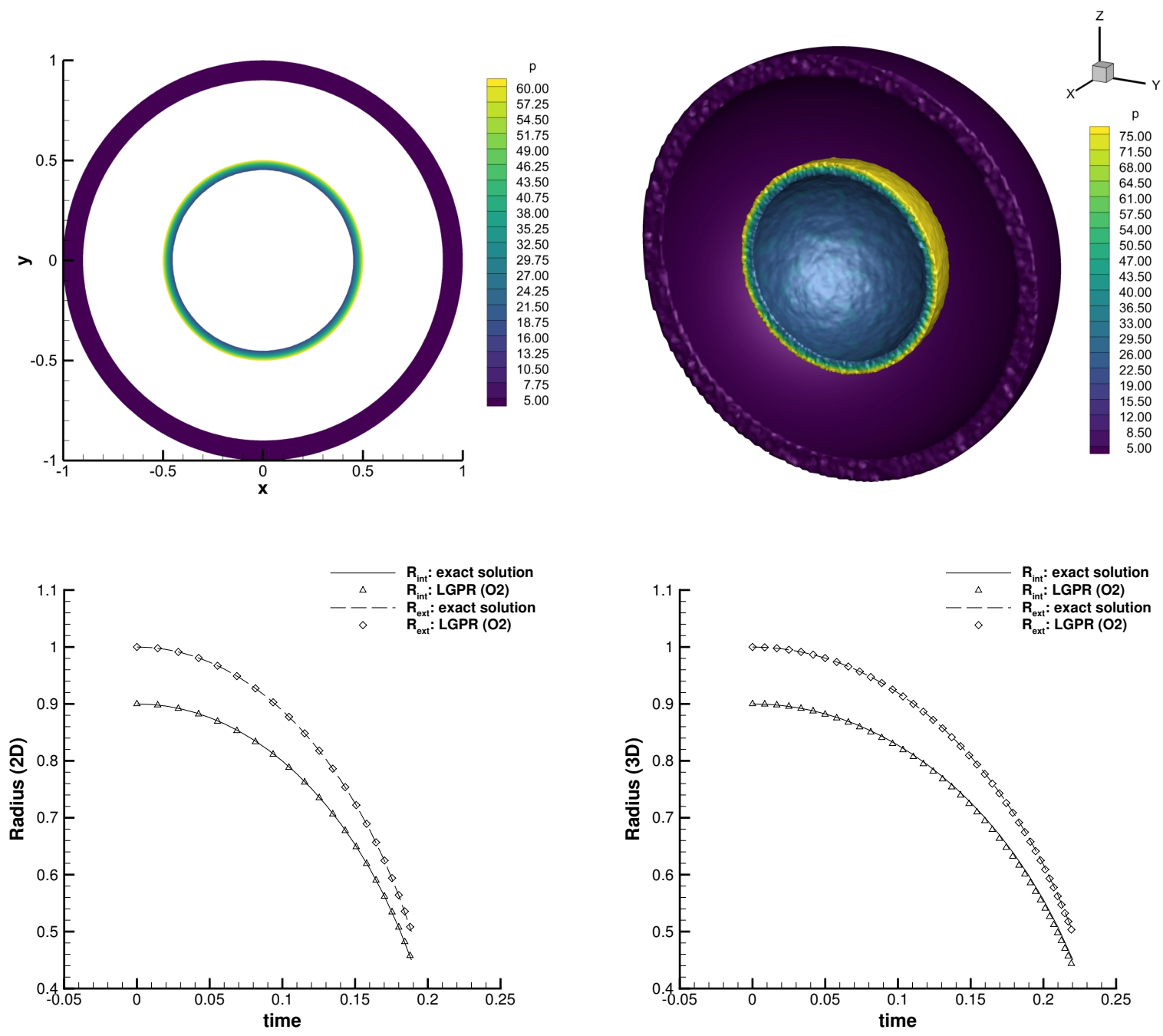

Figure 3: Kidder problem in 2D (left) and in 3D (right). Top: pressure distribution and geometry configuration at the initial and final time of the simulation. Bottom: evolution of the internal $R_{i n t}$ and external $R_{\text {ext }}$ radius of the shell and comparison between analytical and numerical solution. 

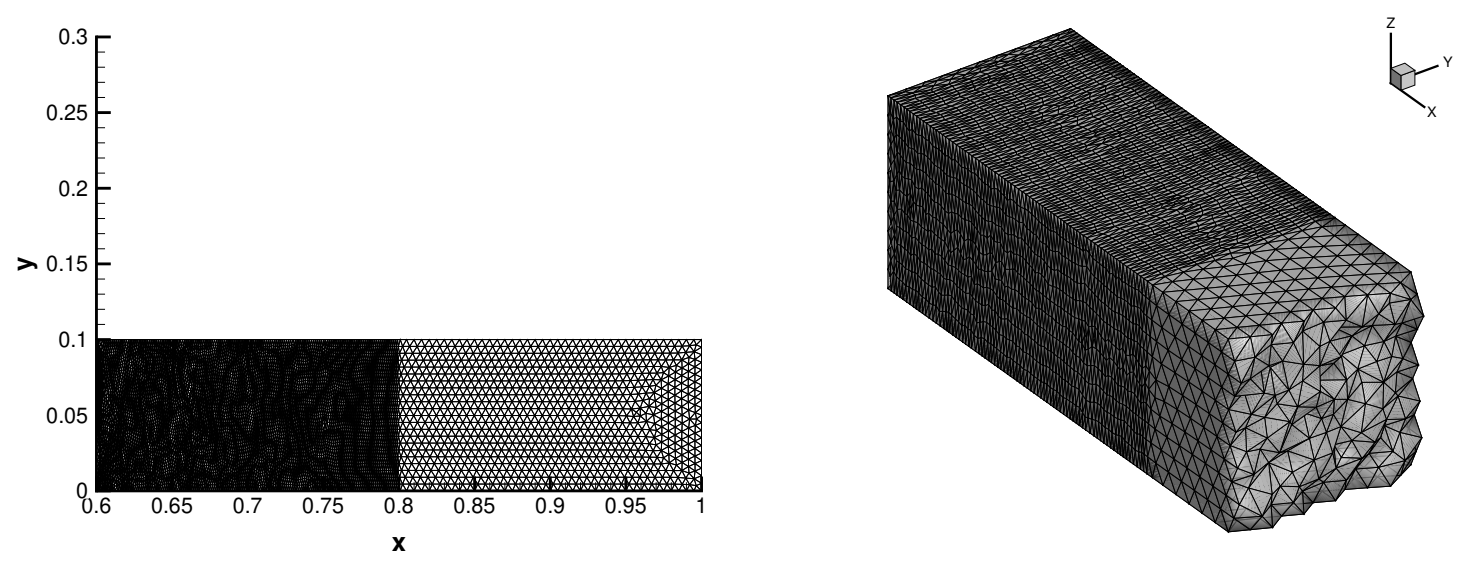

Figure 4: Saltzman problem. Mesh configuration in 2D (left) and in 3D (right) at time $t=0.6$.

order to prevent the generation of invalid elements in those cells lying near the piston that are instantaneously highly compressed for $t>0$. The numerical results are shown in Figure 5 as a scatter plot of all cell values. Compared to the analytical solution [18, 114], a good approximation of the shock plateau and the shock wave location is observed. The decrease of density near the piston, especially in 3D, is due to the well known wall-heating problem, see [113].

\subsection{Sedov problem}

The Sedov problem is concerned with the evolution of a blast wave with cylindrical or spherical symmetry, generated at the origin $\mathbf{O}=(x, y, z)=(0,0,0)$ of the initial computational domain $\Omega(0)=[0 ; 1.2]^{d}$. Symmetry boundary conditions are imposed on those faces which share the origin, while the remaining sides are treated as slip-walls. The characteristic mesh size is $h=1 / 60$ in 3D, whereas we use two different computational meshes in $2 \mathrm{D}$, namely with $h=1 / 40$ and $h=1 / 80$. The ideal gas with $\gamma=1.4$ is initially at rest with an initial pressure of $p=10^{-6}$ in the entire computational domain, apart in those cells containing the origin $\mathbf{O}$ where the pressure is prescribed as

$$
p_{o r}=(\gamma-1) \rho_{0} \frac{E_{t o t}}{\alpha V_{o r}} \quad \text { with } \quad E_{t o t}=\left\{\begin{array}{l}
0.244816 \text { if } d=2 \\
0.851072 \text { if } d=3
\end{array},\right.
$$

with $V_{\text {or }}$ denoting the volume of the elements attached to the origin. The factor $\alpha$ takes into account the cylindrical or spherical symmetry and is set to $\alpha=4$ and $\alpha=8$, respectively. An analytical solution can be derived from selfsimilarity arguments [68], making this test widely used in literature [79]. Figure 6 collects the results for all three simulations, plotting the final mesh configuration as well as a scatter plot of the cell density compared against the exact solution. The shock wave is correctly captured by the conservative LGPR scheme, and a very good symmetry of the density distribution can be appreciated. The density peak is well retrieved thanks to the Lagrangian nature of the scheme, which introduces much less numerical viscosity compared to Eulerian schemes on fixed grids at the same order of accuracy.

\subsection{Riemann problems with viscous fluids}

The test cases presented so far deal with the stiff hydrodynamics limit of the GPR model (2). Here, we want to test the new LGPR scheme for the simulation of viscous fluids using the same set of equations. To this purpose, we solve in a two-dimensional setting the well-known Sod shock tube problem, which is a classical one-dimensional test problem that involves a rarefaction wave traveling towards the left boundary as well as a right-moving contact discontinuity 

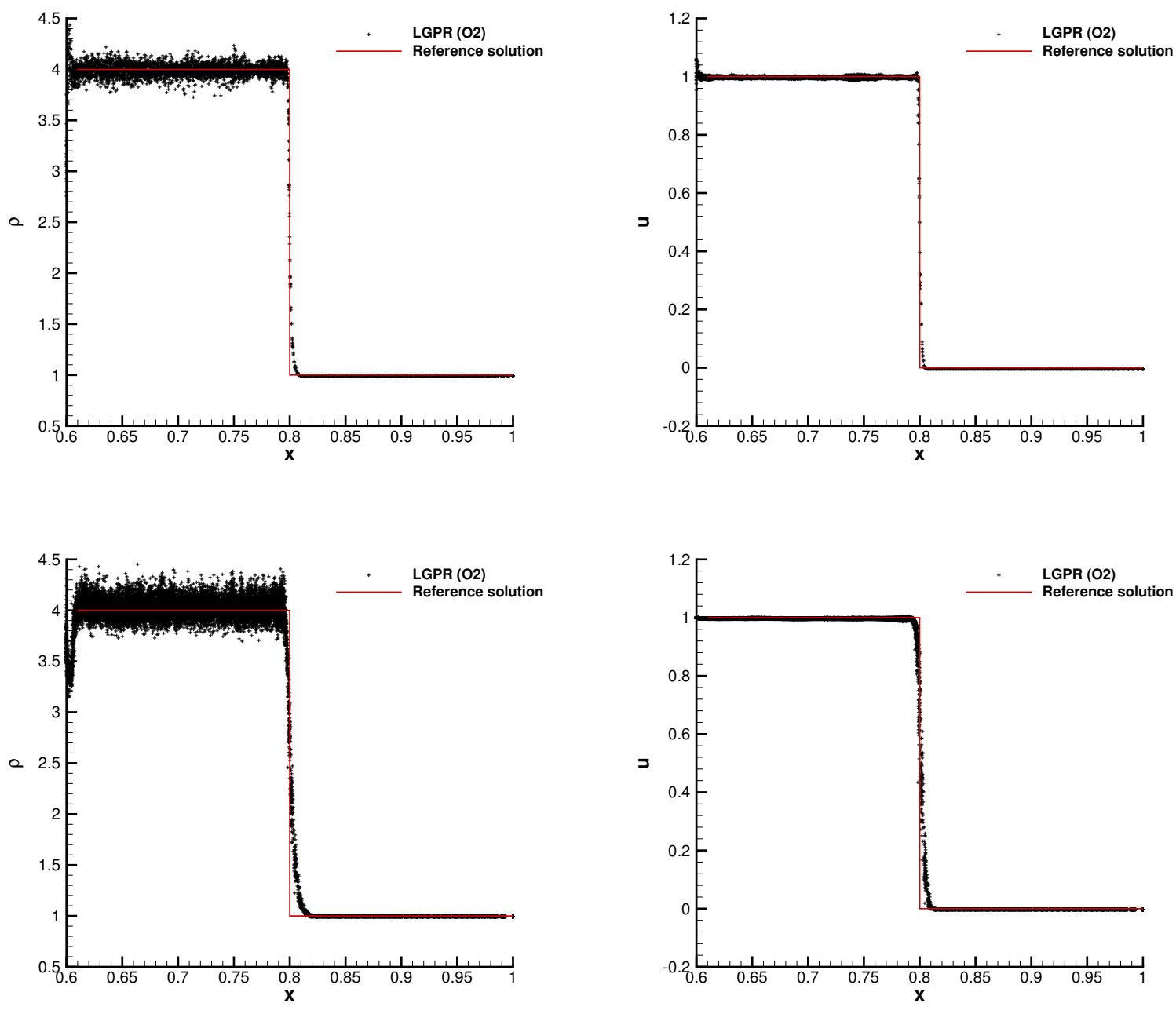

Figure 5: Saltzman problem in 2D (top) and in 3D (bottom). Scatter plot of cell density (left) and horizontal velocity (right) compared against the reference solution at time $t=0.6$. 

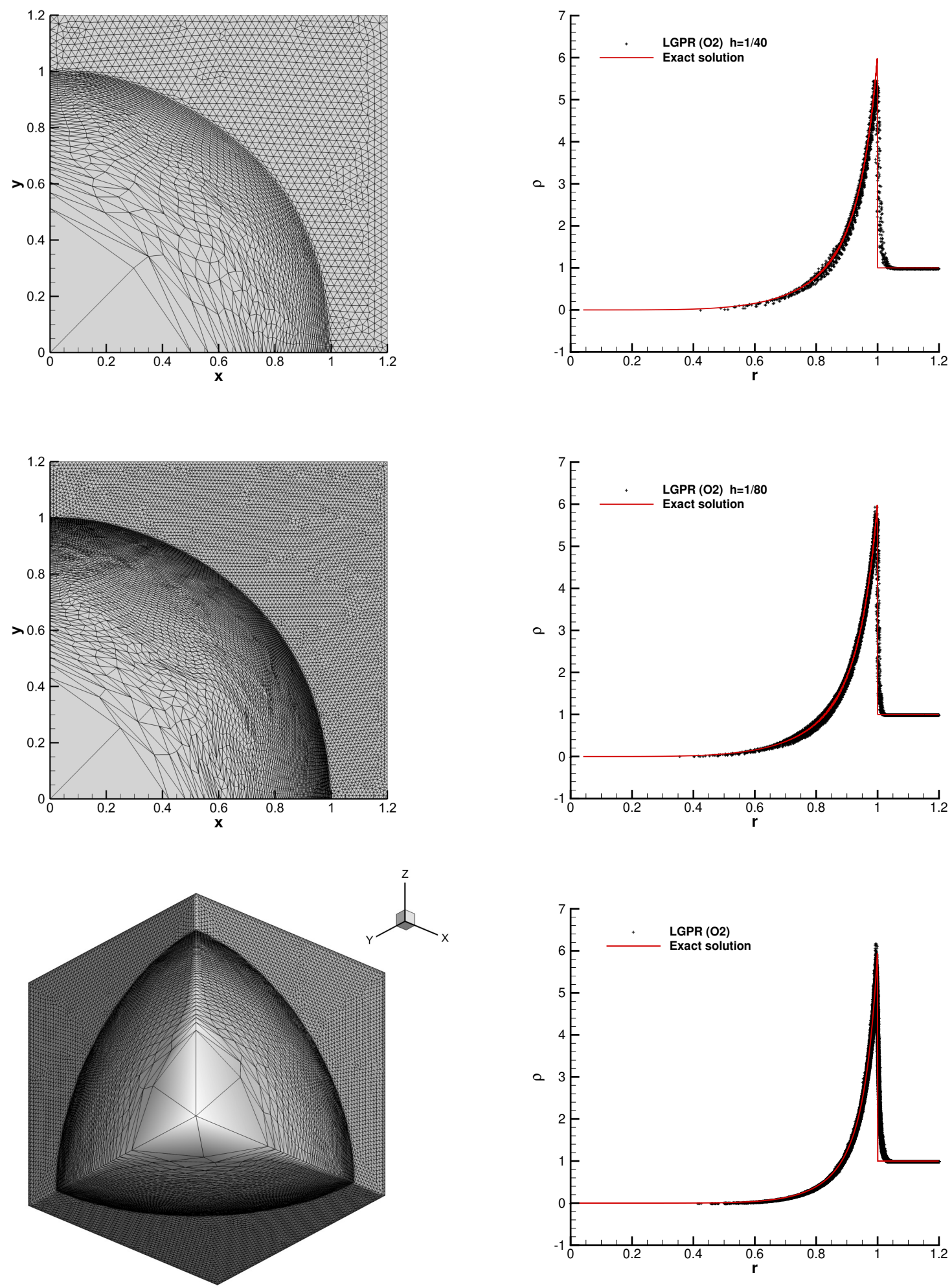

Figure 6: Sedov problem. Mesh configuration (left) and scatter plot of cell density (right) at the final time $t=1$ obtained with mesh size $h=1 / 40$ in $2 \mathrm{D}$ (top), $h=1 / 80$ in $2 \mathrm{D}$ (middle) and $h=1 / 60$ in $3 \mathrm{D}$ (bottom). 
and a shock wave traveling to the right. However, instead of ideal fluids, viscous ones are now considered, with three different viscosity coefficients, namely $\mu=10^{-3}, \mu=5 \cdot 10^{-3}$ and $\mu=10^{-2}$. The initial computational domain is the rectangular box $\Omega(0)=[0 ; 1] \times[0 ; 0.1]$ which is discretized with a characteristic mesh size of $h=1 / 200$. Slip-wall boundaries are set everywhere, hence prescribing zero normal velocity in the nodal solver. The final time is $t_{f}=0.2$ so that all waves remain bounded in the computational domain. The initial condition consists in a discontinuity located at $x_{0}=0.5$ between the left and the right state:

$$
(\rho(x), u(x), p(x))=\left\{\begin{array}{ll}
(1,0,1), & \text { if } \quad x \leq x_{0}, \\
(0.125,0,0.1), & \text { if } \quad x>x_{0} .
\end{array}, \quad t=0 .\right.
$$

The fluid is initially at rest and the ideal gas EOS is adopted with $\gamma=1.4$, while thermal conduction is neglected. According to the asymptotic analysis presented in Section 4, the viscous stress tensor of the Navier-Stokes equation should be retrieved, therefore the results of the LGPR scheme are compared with a reference solution of the NavierStokes equations without thermal conduction computed on a very fine one-dimensional mesh of 10000 cells with a MUSCL-TVD finite volume method. Figure 7 plots a three-dimensional view of the density distribution as well as the mesh configuration across the contact discontinuity at the final time, whereas Figure 8 shows a scatter plot for density, horizontal velocity and pressure of the numerical solution compared against the reference solution of the NavierStokes model. An excellent agreement is obtained for all different values of the viscosity coefficient, demonstrating that the novel LGPR scheme is capable of simulating viscous fluids as well.
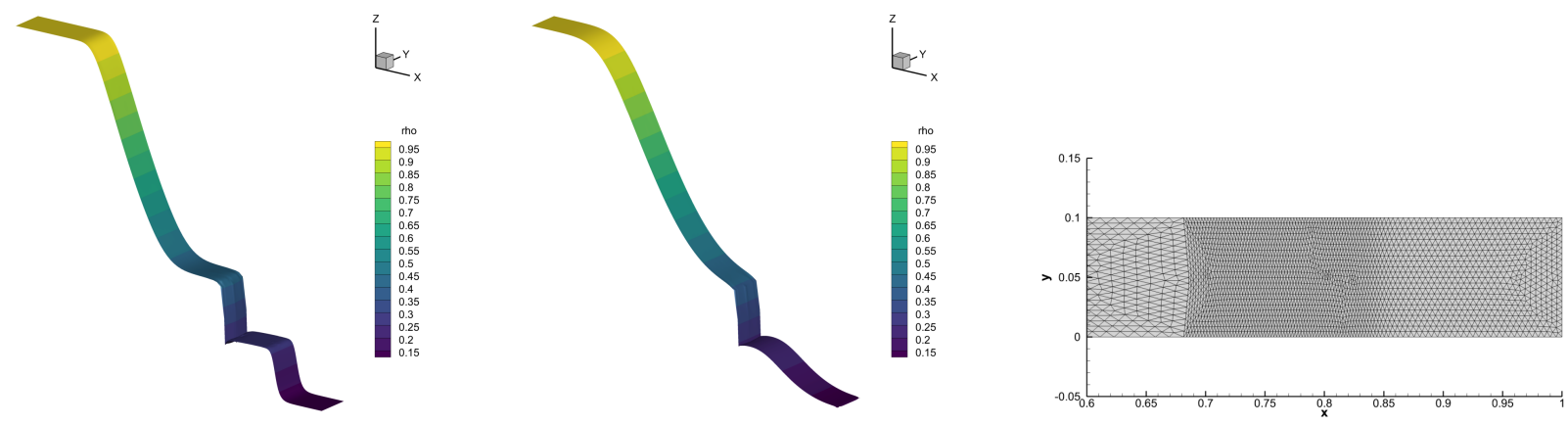

Figure 7: Riemann problems with viscous fluids. Three-dimensional density distribution at the final time $t=0.2$ with viscosity coefficient $\mu=10^{-3}$ (left) and $\mu=10^{-2}$ (middle). A zoom in view of the mesh configuration across the contact discontinuity for $\mu=10^{-3}$ (right).

\subsection{Heat conduction in a gas}

The aim of this test case is to verify the correct behavior of the novel LGPR scheme in the case of a problem dominated by heat transfer via heat conduction. A high density circle of gas is initialized at the center of the computational domain $\Omega(0)=[-0.5,0.5]^{2}$, that is

$$
\rho(0, r)= \begin{cases}2 & r \leq R_{0} \\ 0.5 & x>R_{0}\end{cases}
$$

with $r=\sqrt{x^{2}+y^{2}}$ representing the generic radial coordinate and $R_{0}=0.2$ being the radius of the circle containing the high density gas. The fluid is initially at rest $(u=v=w=0)$ with constant pressure $p=1$ and obeys an ideal gas law with $\gamma=1.4$. The heat wave velocity and heat conduction coefficient are specified in Table 2 . The computational domain is discretized with a characteristic mesh size of $h=1 / 100$ and slip-wall boundaries are imposed on all sides. Figure 9 shows the pressure distribution as well as the metric tensor components $\left(\boldsymbol{G}_{e_{11}}, \mathbf{G}_{e_{12}}\right)$ at the final time. Despite the highly unstructured and non-symmetric mesh shown in Figure 9, the numerical solution exhibits excellent symmetry. Finally, the solution along a 1D cut with 200 points along the $x$-direction at $y=0$ is compared against the reference solution of the Navier-Stokes-Fourier equations for temperature and the heat flux, achieving an excellent agreement. This demonstrates that the stiff limit of the heat conduction equation (2d) is properly retrieved by the LGPR scheme (35d), hence giving numerical evidence of the asymptotic preserving property of the scheme studied in Section 4. 

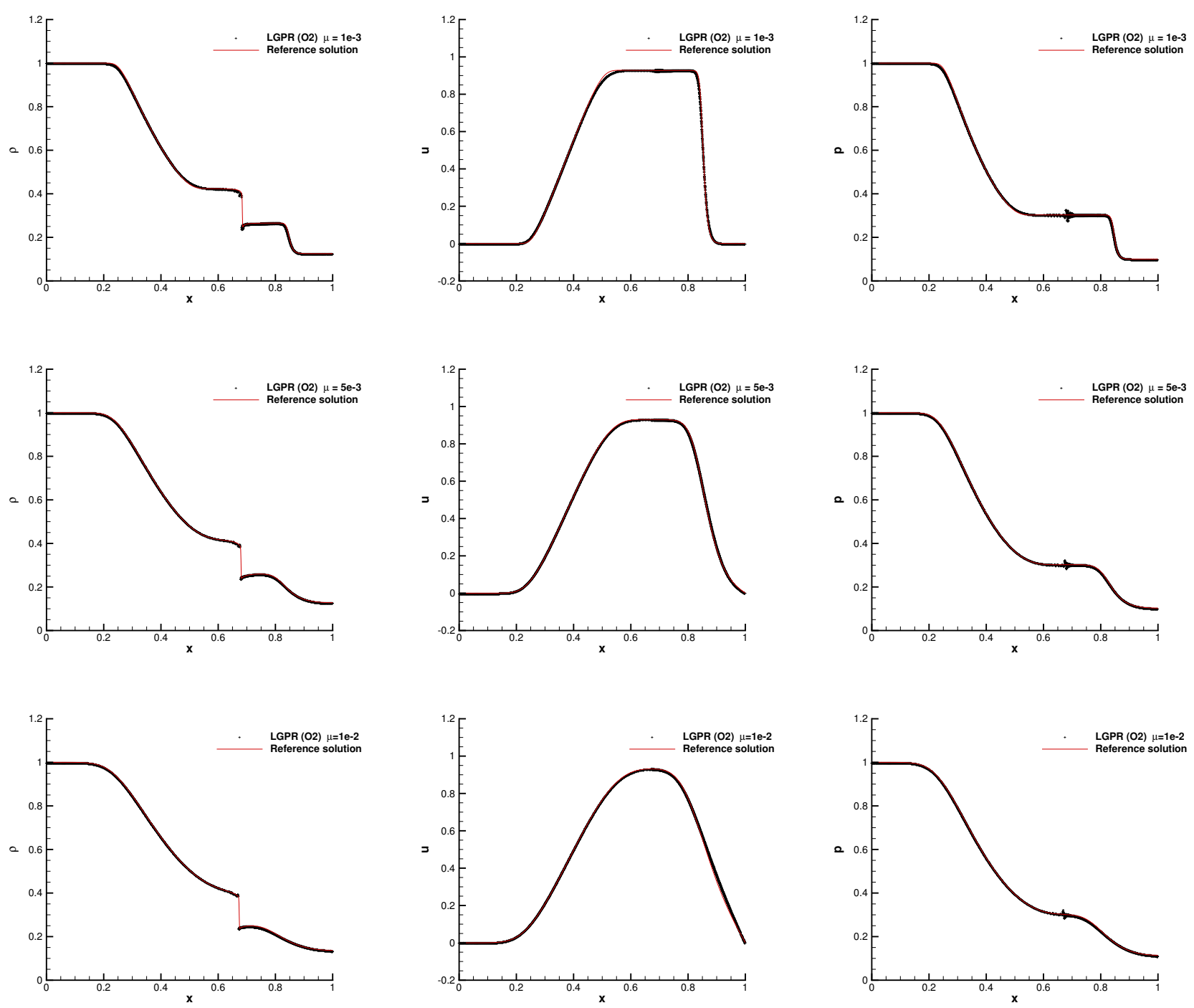

Figure 8: Viscous shock tube problem. Scatter plot of cell density (left), horizontal velocity (middle) and pressure (right) at the final time $t=0.2$ with viscosity coefficient $\mu=10^{-3}$ (top), $\mu=5 \cdot 10^{-3}$ (middle) and $\mu=10^{-2}$ (bottom). Comparison against the reference solution of the Navier-Stokes equations (solid red line). 

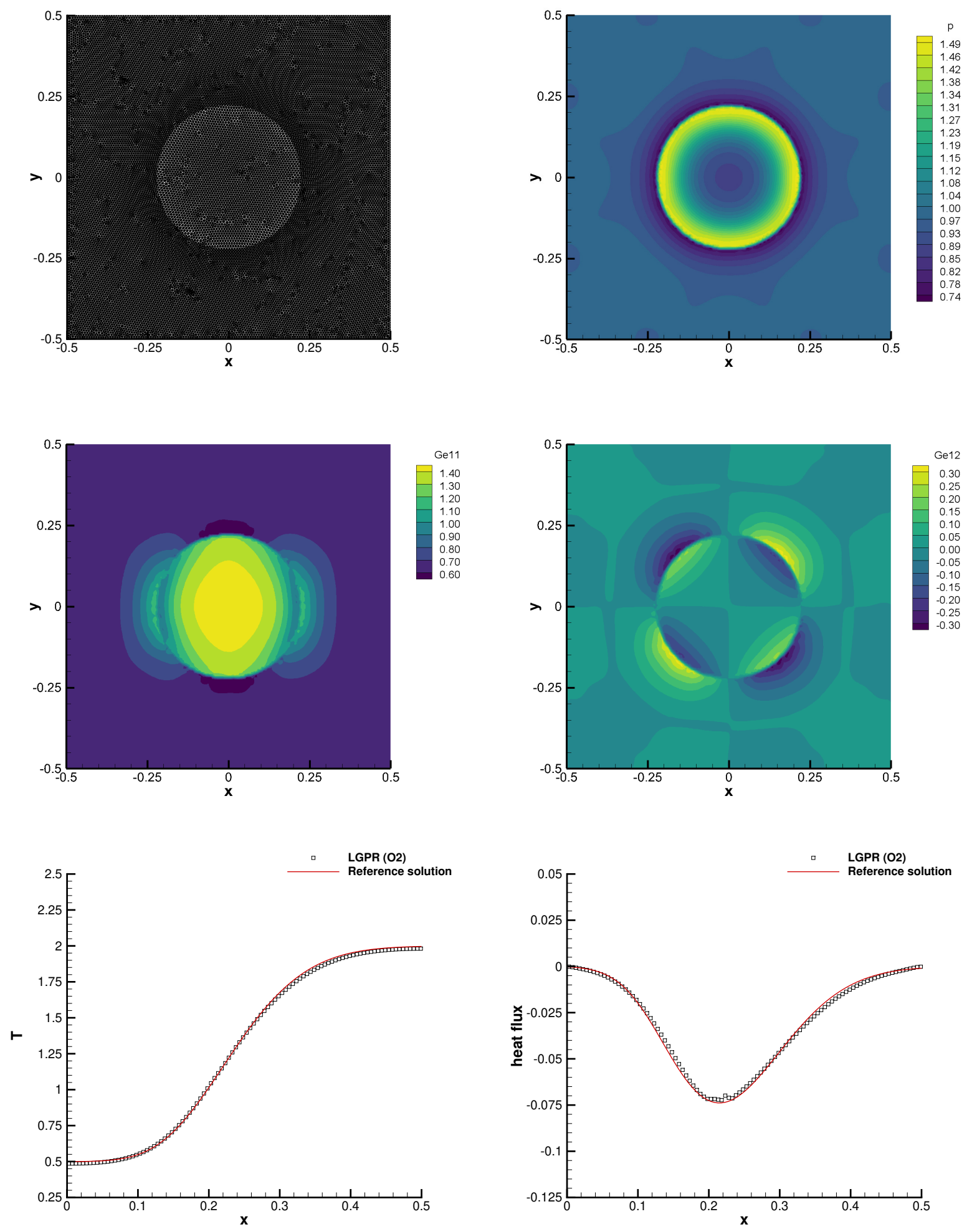

Figure 9: Heat conduction in a gas. Top: mesh configuration (left) and pressure distribution (right) Initial (left) at time $t=1$. Middle: tensor component $\boldsymbol{G}_{e_{11}}$ (left) and $\boldsymbol{G}_{e_{12}}$ (right). Bottom: temperature distribution (left) and heat flux (right). For the Navier-Stokes solution, the classical Fourier heat flux $q_{1}=-\kappa T_{x}$ is shown, while for the GPR model, we plot $q_{1}=\alpha^{2} T J_{1}$. 


\subsection{Viscous shock profile}

In order to verify the numerical method against supersonic viscous flows, we propose to solve the problem of an isolated viscous shock wave which is traveling into a viscous heat conducting fluid at rest with a shock Mach number of $M_{s}=2$. The analytical solution can be found in [7], where the compressible Navier-Stokes-Fourier equations are solved for the special case of a stationary shock wave at Prandtl number $\operatorname{Pr}=0.75$ with constant viscosity. The exact solution for the dimensionless velocity $\bar{u}=\frac{u}{M_{s} c_{0}}$ of this stationary shock wave with shock Mach number $M_{s}$ is then given by the root of the following equation, see [7]:

$$
\frac{|\bar{u}-1|}{\left|\bar{u}-\kappa^{2}\right|^{\kappa^{2}}}=\left|\frac{1-\kappa^{2}}{2}\right|^{\left(1-\kappa^{2}\right)} \exp \left(\frac{3}{4} \operatorname{Re}_{s} \frac{M_{s}^{2}-1}{\gamma M_{s}^{2}} x\right),
$$

with

$$
\kappa^{2}=\frac{1+\frac{\gamma-1}{2} M_{s}^{2}}{\frac{\gamma+1}{2} M_{s}^{2}} .
$$

Equation (107) allows the dimensionless velocity $\bar{u}$ to be obtained as a function of $x$. The form of the viscous profile of the dimensionless pressure $\bar{p}=\frac{p-p_{0}}{\rho_{0} c_{0}^{2} M_{s}^{2}}$ is given by the relation

$$
\bar{p}=1-\bar{u}+\frac{1}{2 \gamma} \frac{\gamma+1}{\gamma-1} \frac{(\bar{u}-1)}{\bar{u}}\left(\bar{u}-\kappa^{2}\right) .
$$

Finally, the profile of the dimensionless density $\bar{\rho}=\frac{\rho}{\rho_{0}}$ is derived from the integrated continuity equation: $\bar{\rho} \bar{u}=1$. To obtain an unsteady shock wave traveling into a medium at rest, it is sufficient to superimpose a constant velocity field $u=M_{s} c_{0}$ to the solution of the stationary shock wave found in the previous steps. The initial computational domain is the rectangular channel $\Omega(0)=[0,1] \times[0,0.2]$ which is paved with two different triangular meshes of characteristic mesh size $h=1 / 100$ and $h=1 / 200$. On the left side of the domain $(x=0)$ the constant inflow velocity is prescribed, whereas periodic boundaries are set along the $y$ direction and a constant pressure is imposed at $x=1$. The initial condition involves a shock wave centered at $x=0.25$ propagating at Mach $M_{s}=2$ from left to right with a Reynolds number of $R e=100$. The polytropic index of the ideal gas is $\gamma=1.4$ and the upstream shock state is defined by $\rho=1$, $u=v=0, p=1 / \gamma$. Figure 10 shows the initial and final mesh configuration at time $t_{f}=0.2$ with the shock front located at $x=0.65$.

Figure 11 illustrates a comparison against the analytical solution at the final time, where one can note an excellent matching. We compare the exact solution and the numerical solution, extracted as a 1D cut with 200 points along the $x$-direction at $y=0.1$, for density, horizontal velocity, pressure and heat flux. Mesh convergence is also qualitatively demonstrated by the numerical results obtained with $h=1 / 100$ and $h=1 / 200$. Finally, the heat flux and the viscous stress component $\sigma_{11}$ are depicted in Figure 12 and compared against the Navier-Stokes-Fourier model, where the Navier-Stokes stress tensor is recovered in the stiff limit by the LGPR scheme, as proven in Section 4.

\subsection{Collapse of a thick-walled cylindrical beryllium shell}

The next test case aims at exploiting the capability of the GPR model (2) to simulate elasto-plastic solids. We consider a test problem firstly proposed in [63], which describes the collapse of a cylindrical beryllium shell responding to an initial radial velocity field directed towards its center. The initial setup is taken from [67], thus the computational domain $\Omega(0)$ is the shell with inner and outer radii $r_{i n t}=8 \times 10^{-2}$ and $r_{\text {ext }}=10 \times 10^{-2}$. Free-traction boundary conditions are considered everywhere. The material is beryllium and the Mie-Grüneisen equation of state (6) is used with parameters $\Gamma_{0}=1.16$ and $s=1.124$. The adiabatic sound speed results to be $c_{0}=12870$. The initial pressure is $p=0$ and the radial velocity magnitude is given by $v_{r}(0, r)=-V_{0}\left(\frac{r_{i n t}}{r}\right)^{2}$, with $r=\sqrt{x^{2}+y^{2}}$. According to [1], three different values of $V_{0}$ are considered, namely $V_{0}^{(1)}=417.1, V_{0}^{(2)}=454.7$ and $V_{0}^{(3)}=490.2$. The corresponding final times are $t_{f}^{(1)}=125.67 \cdot 10^{-6}, t_{f}^{(2)}=131.6 \cdot 10^{-6}$ and $t_{f}^{(3)}=136.26 \cdot 10^{-6}$. The initial kinetic energy due to the velocity distribution is entirely dissipated by the plastic deformation of the material leading to a deceleration of the shell. Therefore, in [63] a closed form solution at the stopping time is derived under the ideal plasticity assumption, which leads to a relationship between the initial velocity $V_{0}$ and the inner and outer stopping radii. In this test case, the 

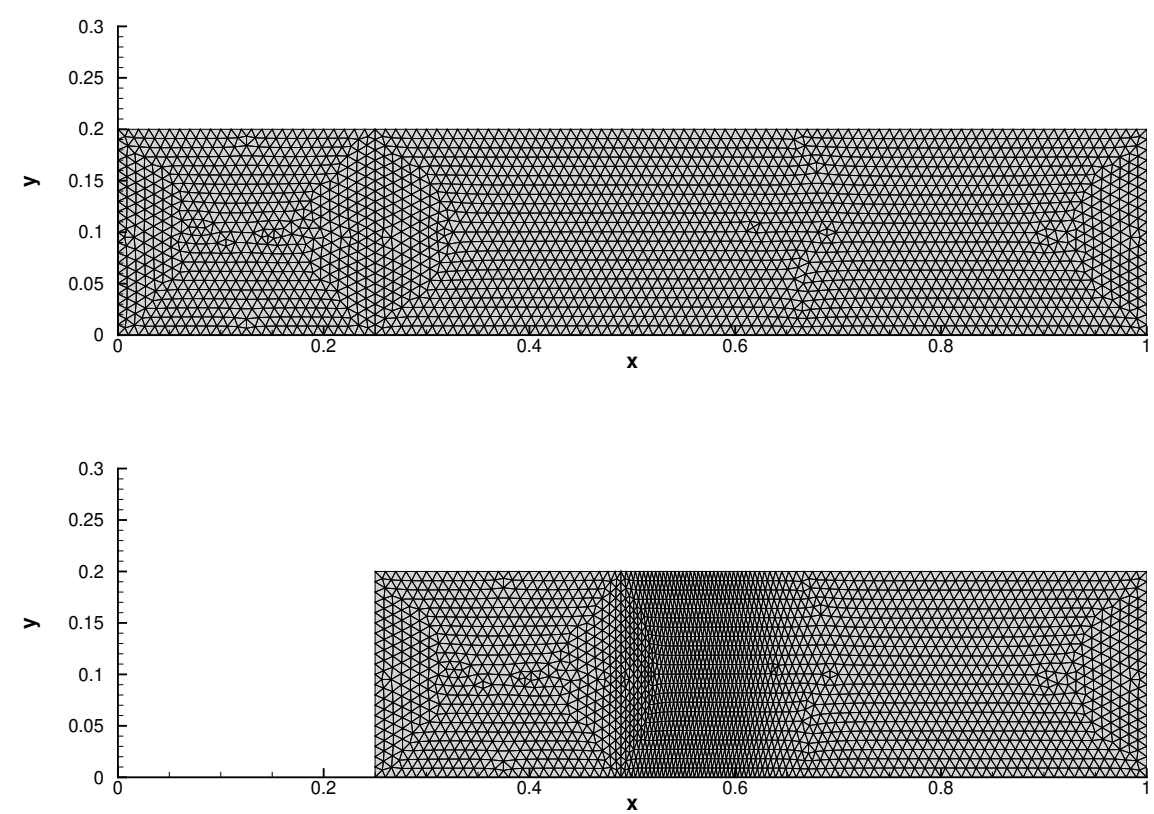

Figure 10: Viscous shock profile. Initial (left) and final (right) mesh configuration with $h=1 / 100$

material experiences elasto-plastic deformations, thus the closure relations (12) are adopted to account for plasticity effects. Specifically, the yield strength is $\sigma_{Y}=330 \cdot 10^{6}$ and the parameters of the power law for the computation of the relaxation time $\tau_{1}$ are the exponent $n=12$ and $\tau_{10}=10^{-7}$. The test case is run for all the three velocities $V_{0}$ on two different unstructured meshes with characteristic mesh size of $h=1 / 100$ and $h=1 / 140$, in order to show mesh convergence of the numerical solution. The final mesh configuration for $V_{0}=417.1$ is depicted in Figure 13, while the plastic map $\eta=\sigma / \sigma_{Y}$ and the normalized relaxation time $\tau_{1} / \tau_{10}$ at the final time of each simulation are shown in Figure 14.

The time evolution of the internal and external frontiers of the shell, i.e. $R_{i n t}(t)$ and $R_{e x t}(t)$, is plot in Figure 15 , which is compared against the exact displacement of the shell at the final time of each simulation. A good agreement can be observed and the finer mesh correctly retrieves a more accurate solution. We underline that the usage of a high quality, though unstructured, computational mesh is crucial for maintaining the symmetry of the numerical solution as demonstrated by Figure 14. Finally, the analysis of energy conservation over time is plot in Figure 15, where we report the volumetric and shear internal energy $\left(E_{h}\right.$ and $E_{e}$, respectively), kinetic $\left(E_{k}\right)$ and total $(E)$ energy. We clearly see that all initial kinetic energy is dissipated into internal energy due to elasto-plastic deformations that occur in the material. Indeed, the volumetric energy contribution is rather small compared to the shear internal energy $E_{e}$.

\section{9. $2 D$ projectile impact}

This problem consists of the impact of a two-dimensional aluminum bar impacting on a rigid wall and the setup is taken from [82]. The computational domain is the initial projectile $\Omega(0)=[0 ; 5] \times[0 ; 1]$ that is paved with two different triangular meshes of characteristic size $h=1 / 100$ and $h=1 / 200$. A slip-wall boundary is set on the left side of the domain, while free-traction boundary conditions are imposed elsewhere. The final time of the simulation is $t_{f}=0.005$ and the material undergoes plastic deformations that convert the initial kinetic energy into shear internal energy. The closure relation for the hydrodynamics part of the energy is the Mie-Grüneisen EOS with $\Gamma_{0}=2$ and $s=1.338$, while the relaxation time $\tau_{1}$ is computed relying on the formulation (12) with $n=10$ and $\tau_{10}=5 \cdot 10^{-4}$. The yield stress for aluminum is $\sigma_{Y}=300 \cdot 10^{6}$ and the adiabatic sound speed is $c_{0}=5328$. Initially, the material 

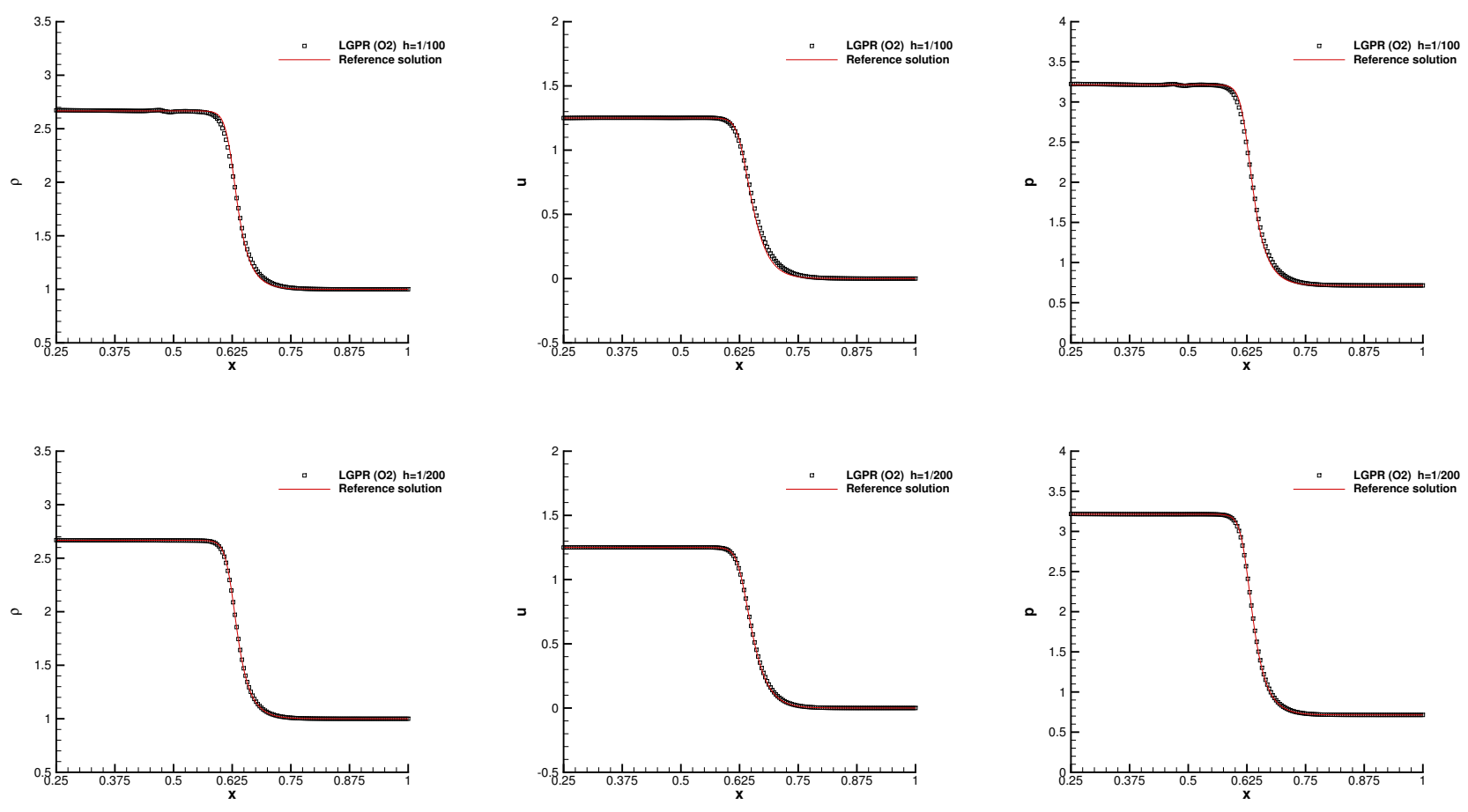

Figure 11: Viscous shock profile for the Mach number $M_{s}=2$. Comparison of the exact solution of the compressible Navier-Stokes equations with the Lagrangian GPR model for density (left), horizontal velocity (middle) and pressure (right) with mesh size $h=1 / 100$ (top) and $h=1 / 200$ (bottom). 

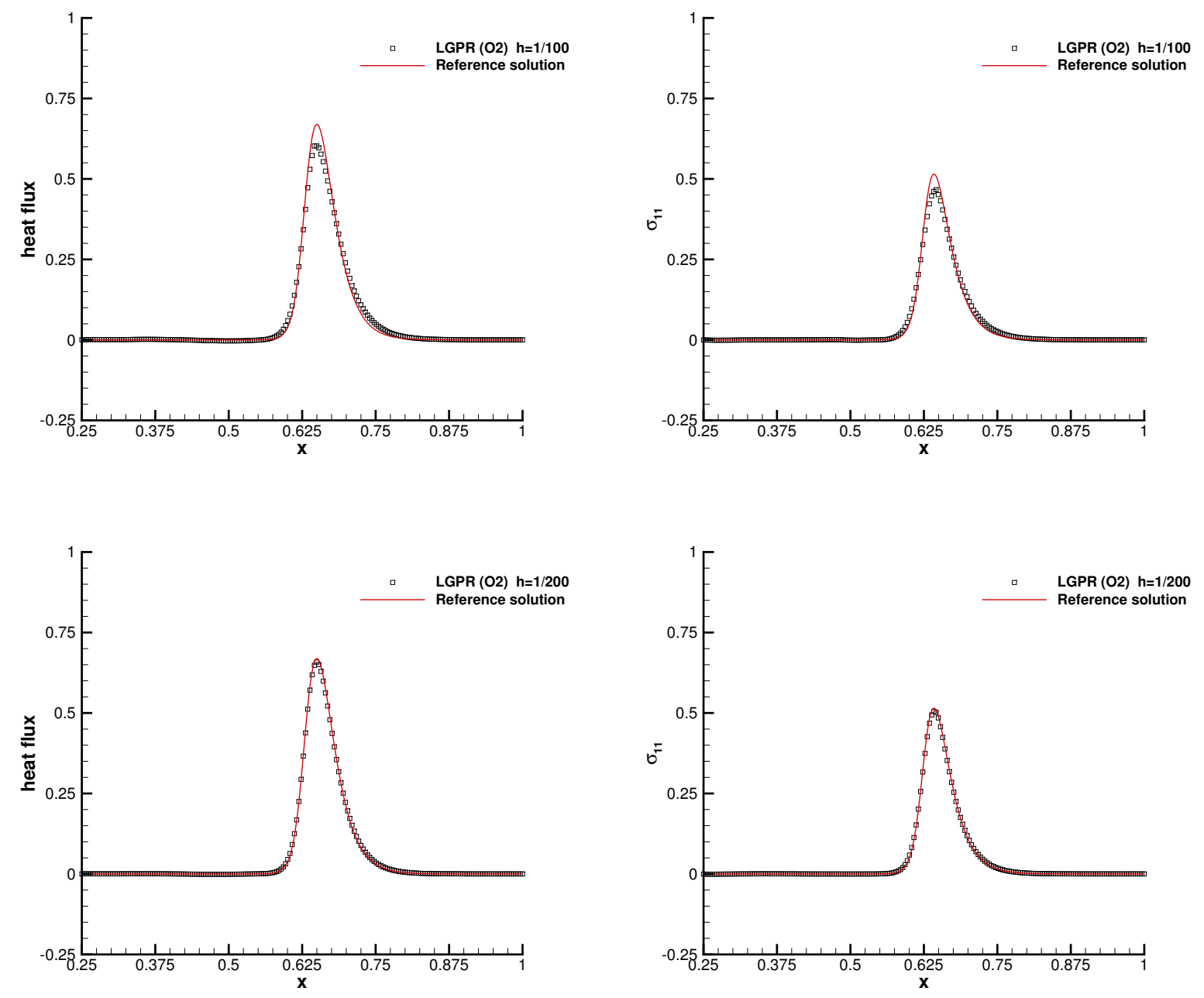

Figure 12: Viscous shock profile. Comparison of the exact solution of the compressible Navier-Stokes equations with the Lagrangian GPR model for viscous stress tensor component $\sigma_{11}$ (left) and heat flux (right) with mesh size $h=1 / 100$ (top) and $h=1 / 200$ (bottom). For the Navier-Stokes solution, the classical Fourier heat flux $q_{1}=-\kappa T_{x}$ is shown, while for the GPR model, we plot $q_{1}=\alpha^{2} T J_{1}$. 

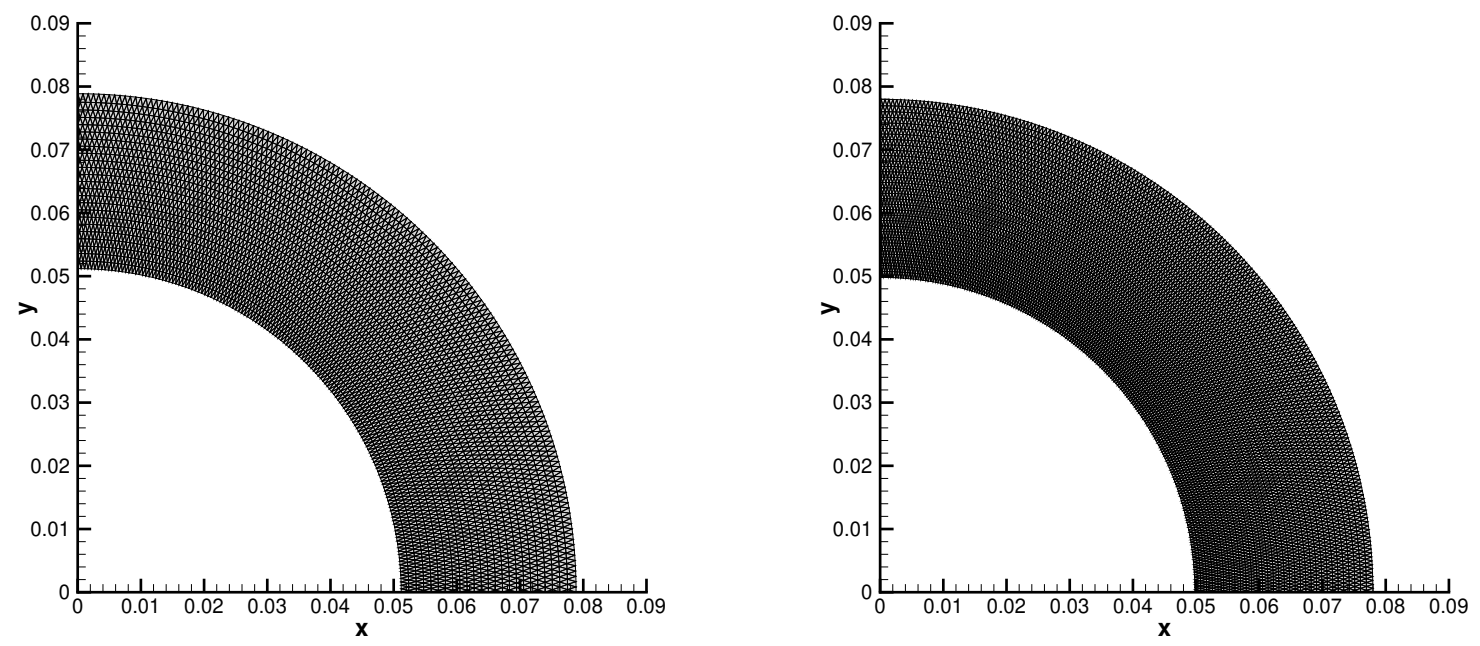

Figure 13: Collapse of beryllium shell for the test cases with $V_{0}^{(1)}=417.1$ (left). Final mesh configuration at time $t_{f}=125.67 \cdot 10^{-6}$ with mesh size $h=1 / 100$ (left) and $h=1 / 140$ (right).
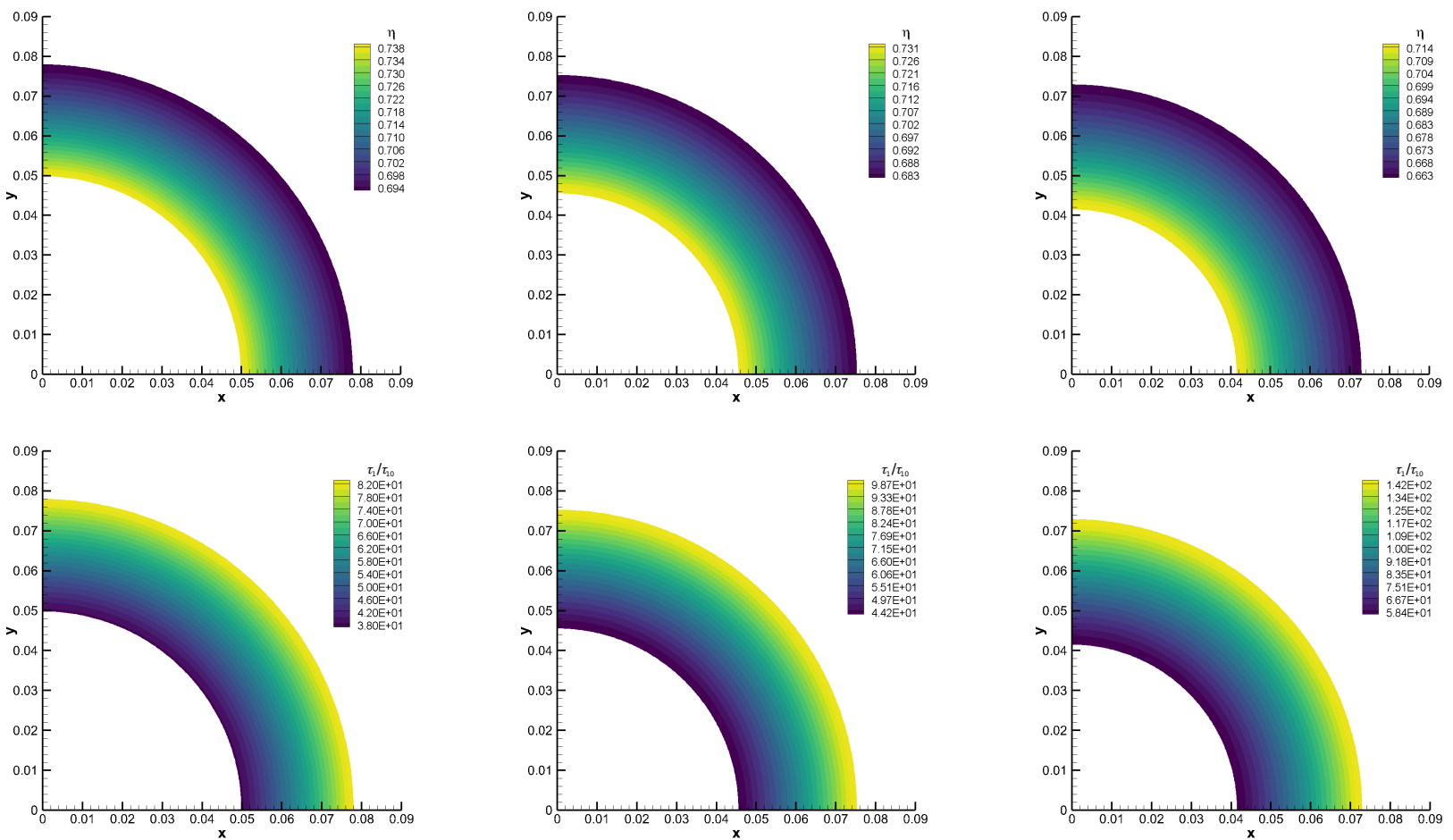

Figure 14: Collapse of beryllium shell for the three test cases: $V_{0}^{(1)}=417.1$ (left), $V_{0}^{(2)}=454.7$ (middle) and $V_{0}^{(3)}=490.2$ (right) with mesh size $h=1 / 140$. Numerical distribution of plastic map $(\eta)$ and normalized relaxation time $\left(\tau_{1} / \tau_{10}\right)$ at the final time of each test case. 

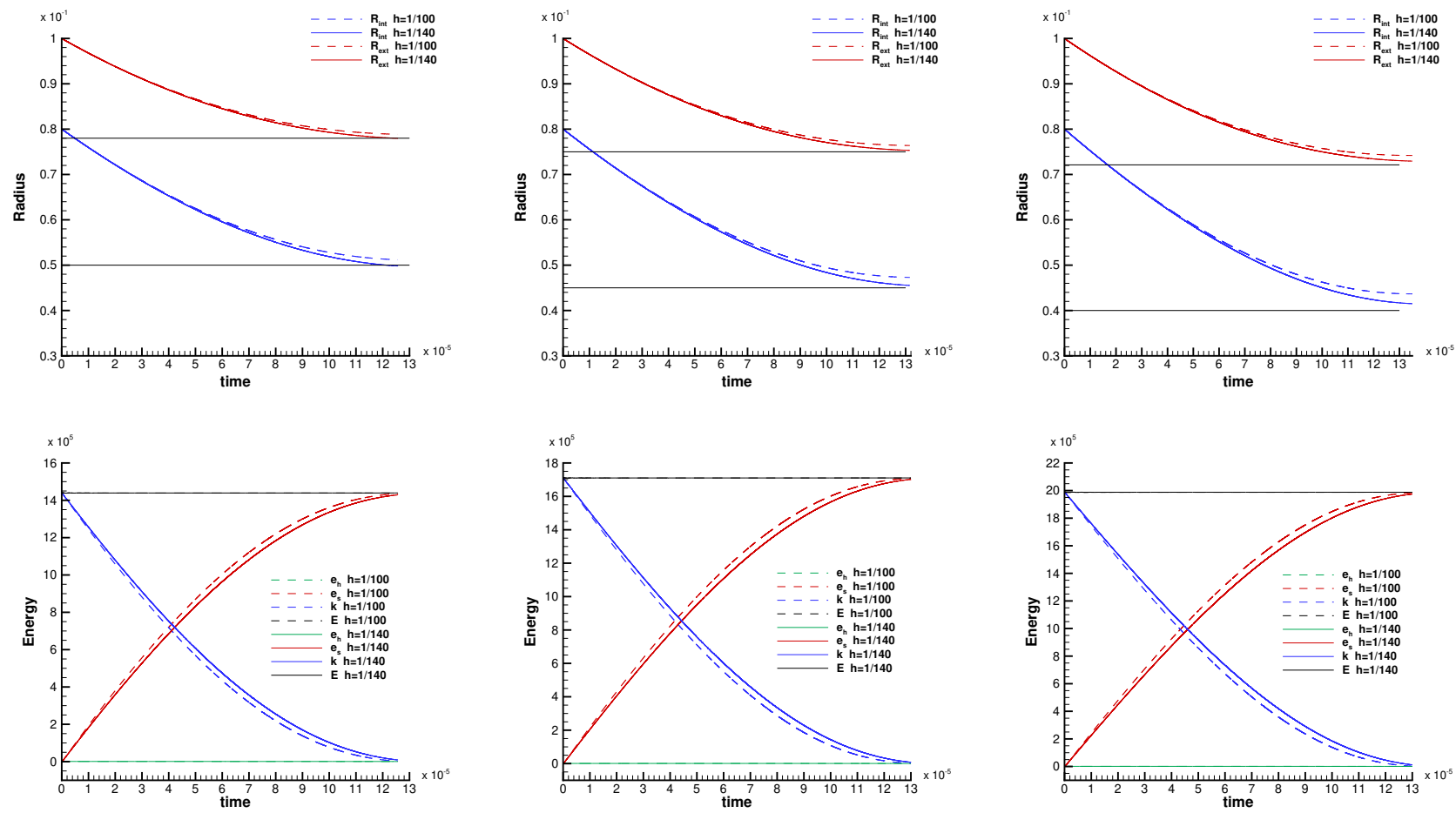

Figure 15: Collapse of beryllium shell for the three test cases: $V_{0}^{(1)}=417.1$ (left), $V_{0}^{(2)}=454.7$ (middle) and $V_{0}^{(3)}=490.2$ (right). Top: time evolution of the internal $R_{\text {int }}$ and external $R_{\text {ext }}$ radius of the shell and comparison between analytical and numerical solution. Bottom: analysis of energy conservation in terms of volumetric and shear internal energy $\left(E_{h}\right.$ and $E_{e}$, respectively), kinetic $\left(E_{k}\right)$ and total $(E)$ energy. Results with mesh size $h=1 / 100$ are drawn with dashed lines, while solid lines refer to mesh size $h=1 / 140$. 
is assigned $p=0$ with a velocity set to $\mathbf{v}=(-150,0,0)$, thus the projectile hits the wall located at the left side of the domain. Although there exists no exact solution for this problem, it is nonetheless employed for verifying robustness and accuracy. Figure 16 depicts the plastic map $\eta=\sigma / \sigma_{Y}$ at different output times as well as the final mesh configuration and the normalized relaxation time $\tau / \tau_{10}$. Plasticity effects are experienced by the material during the impact and the stresses at the end of the simulation visible in Figure 16 are residual stresses. This observation is also confirmed by the energy conservation analysis presented in Figure 17, where all the initial kinetic energy $E_{k}$ is converted into shear internal energy $E_{e}$. Finally, the time evolution of the maximum length of the projectile is depicted, obtaining a final length of approximately $L=4.62$ which is in good agreement with the literature [82, 90]. Mesh convergence is shown as well by comparing the results with $h=1 / 100$ and $h=1 / 200$.
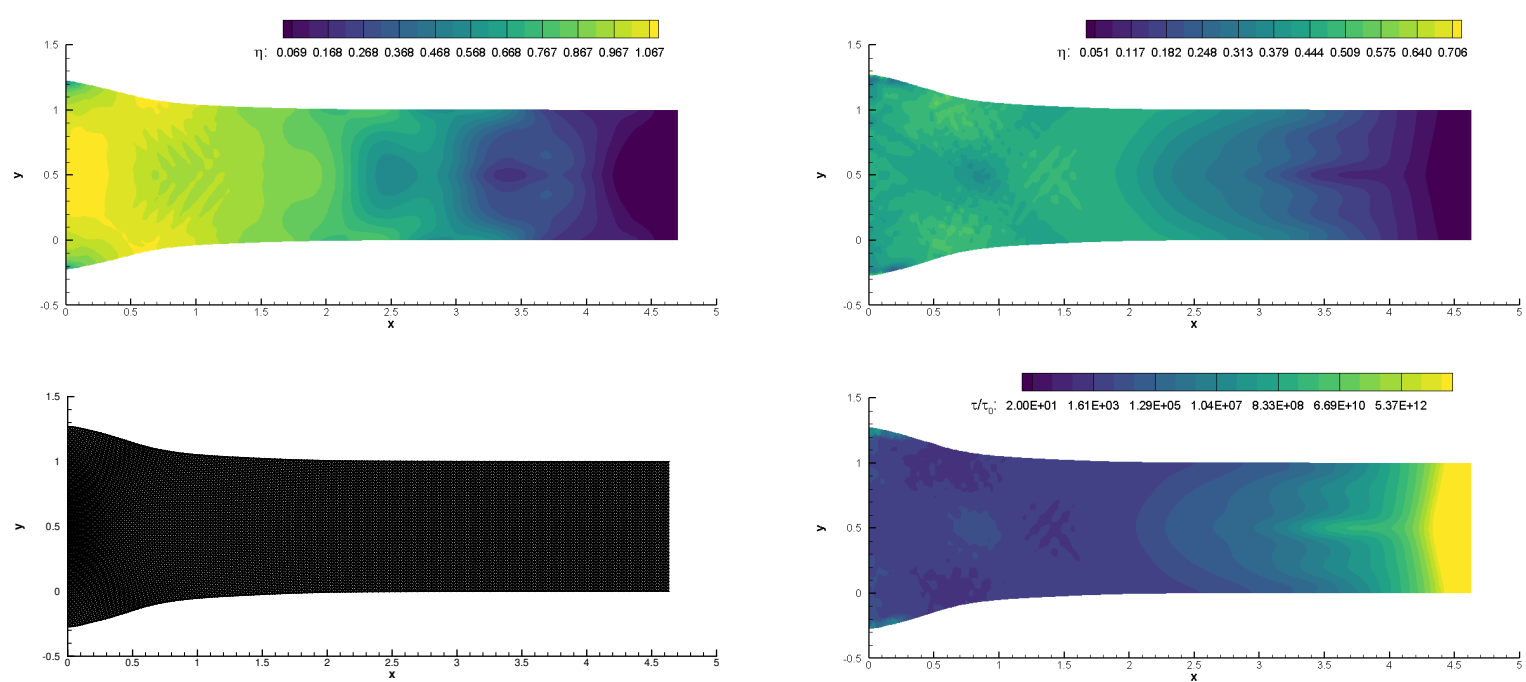

Figure 16: 2D projectile impact. Top: numerical distribution of plastic map $(\eta)$ at time $t=2.5 \cdot 10^{-3}$ (left) and $t=5 \cdot 10^{-3}$ (right). Bottom: numerical distribution of normalized relaxation time $\left(\tau / \tau_{10}\right)$ at the final time $t=5 \cdot 10^{-3}$ (right) and mesh configuration (left).

\subsection{D Taylor bar impact on a wall}

Next, we consider the fully three-dimensional simulation of a copper target that impacts a solid wall, according to the setup provided in [109]. A cylindrical rod made of copper has an initial length $L_{0}=0.0324$ and an initial radius $R_{0}=0.0032$. At $t=0$, it hits a rigid flat plate with velocity $\mathbf{v}(0, \mathbf{x})=(0,0,-227)$ and pressure $p=0$. The computational mesh is composed of $N_{E}=16464$ tetrahedra with characteristic mesh size $h=1 / 50$. Freetraction boundary conditions are set everywhere, apart on the impact surface where wall boundaries are imposed. The simulation is carried on until the final time $t_{f}=80 \cdot 10^{-6}$ and the Mie-Grüneisen EOS is used for copper with $\Gamma_{0}=2, s=1.48$ and yield strength $\sigma_{Y}=400 \cdot 10^{6}$. To take into account plastic deformations, the relaxation time $\tau_{1}$ is dynamically computed with (12) using $n=10$, while we study the different behavior of the material by setting $\tau_{10}=10^{-7}$ and $\tau_{10}=10^{-5}$. The plastic map $\eta$ and the pressure distribution are shown in Figure 18 for $\tau_{10}=10^{-5}$ at time $t=2 \cdot 10^{-5}$ and $t=t_{f}$, highlighting that most of the plasticity effects take place close to the wall at the initial instants of the impact. Figure 19 presents the study of energy conservation over time as well as the time evolution of the length of the copper rod. As $\tau_{10}$ increases, the material behavior gets closer to elastic solids, while a more realistic setting is recovered using $\tau_{10}=10^{-7}$, which correctly accounts for plastic deformations.

\subsection{Elastic vibration of a beryllium plate}

This test case describes the elastic vibration of a beryllium plate or bar, see [90, 27] for instance. Here we consider both the $2 \mathrm{D}$ and the $3 \mathrm{D}$ version. The computational domain is $\Omega^{2 D}(0)=[-0.03 ; 0.03] \times[-0.005 ; 0.005]$ and $\Omega^{3 D}(0)=[-0.03 ; 0.03] \times[-0.005 ; 0.005] \times[-0.005 ; 0.005]$, thus the length of the bar is $L=0.06$. A characteristic 

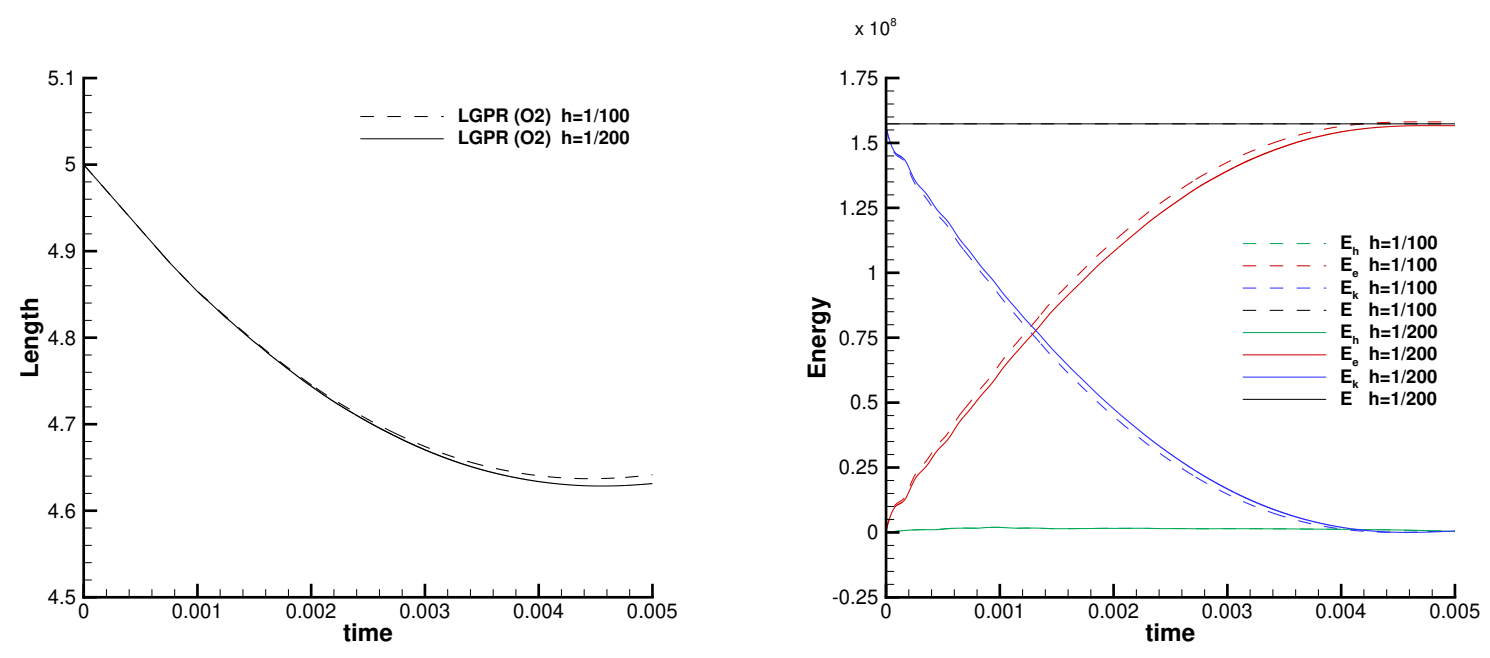

Figure 17: 2D projectile impact. Left: time evolution of the projectile length. Right: analysis of energy conservation in terms of volumetric and elastic energy ( $E_{h}$ and $E_{e}$, respectively), kinetic $\left(E_{k}\right)$ and total $(E)$ energy. Results with mesh size $h=1 / 100$ are drawn with dashed lines, while solid lines refer to mesh size $h=1 / 200$.

mesh size of $h=1 / 200$ is used in 2D, while $h=1 / 100$ is adopted in 3D. Free-traction boundary conditions are set everywhere and the final time of the simulation is chosen to be $t_{f}=3 \cdot 10^{-5}$, so that approximately one oscillating period is completed. The hydrodynamics part of the energy is given by the Neo-Hookean EOS (7), with Young modulus $Y=3.1827 \cdot 10^{11}$ and Poisson ratio $v=0.0539$. The material is initially loaded via a perturbed initial velocity field $\mathbf{v}^{2 D}(0, \mathbf{x})=\left(0, V_{0}(x), 0\right)$ and $\mathbf{v}^{3 D}(0, \mathbf{x})=\left(0,0, V_{0}(x)\right)$ of the form

$$
V_{0}(x)=A \omega\left[a_{1}\left(\sinh \left(x^{\prime}\right)+\sin \left(x^{\prime}\right)\right)-a_{2}\left(\cosh \left(x^{\prime}\right)+\cos \left(x^{\prime}\right)\right)\right],
$$

where $x^{\prime}=\alpha(x+L / 2), \alpha=78.834, A=4.3369 \times 10^{-5}, \omega=2.3597 \times 10^{5}, a_{1}=56.6368$ and $a_{2}=57.6455$. In this setting, the LGPR scheme exactly collapses to the cell-centered finite volume scheme recently introduced in [23]. Figure 20 depicts the mesh configuration and the pressure distribution at three different output times during one flexural period, whereas in Figure 21 we show the time evolution of the vertical component of the velocity of the barycenter of the bar, i.e. the mesh point originally located at $\mathbf{x}_{0}=(0,0,0)$. The first and second order schemes are compared, demonstrating that second order accuracy in space and time is responsible of a remarkable reduction of numerical dissipation. The bar dissipates almost all initial kinetic energy for the first order scheme, which is not due to plastic deformations, since the material is a purely elastic solid, but only because of an excessive numerical dissipation in the nodal solver and the face-based fluxes. This demonstrates the advantages induced by a higher order scheme in space and time.

\subsection{Twisting column}

Finally, a highly nonlinear scenario is considered by simulating a twisting column according to the test problem set up in [61] and the references therein. The computational domain is given by an initial unit squared cross section column of height $H=6$, i.e. $\Omega(0)=[-0.5 ; 0.5] \times[-0.5 ; 0.5] \times[0 ; 6]$. The $z=0$ face of the column is embedded into a wall, while the rest of the faces are assigned free-traction boundary conditions. An initial sinusoidal angular velocity field relative to the origin is given by $\mathbf{v}(0, \mathbf{x})=\omega_{0} \sin \left(\pi \frac{z}{2 H}\right)(y,-x, 0)^{t}$, while pressure is set to $p=0$. Two different magnitudes of the angular velocity are considered, namely $\omega_{0}=100$ and $\omega_{0}=200$. The main objective of this problem is to assess the capability of the proposed methodology to deal with the limit of incompressibility. A material with Neo-Hookean hydrodynamics EOS is used with Young modulus $Y=1.7 \cdot 10^{7}$ and Poisson ratio $v=0.45$. The simulation is run until $t_{f}=0.3$. Qualitatively one should observe a severe twist of the column which returns to its initial position. Driven by its own inertia, the bar keeps twisting until the final time. The mesh of the 


$$
\frac{\mathbb{I}}{\mathbb{I}}
$$



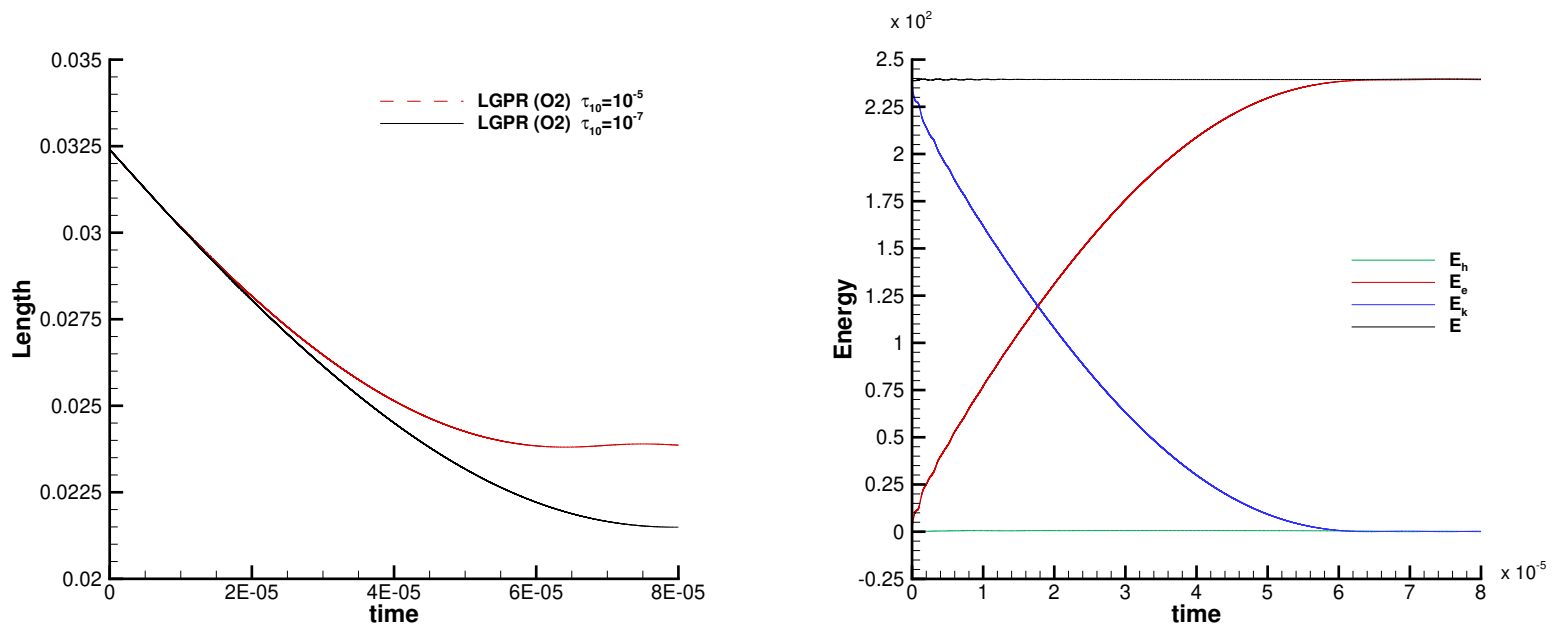

Figure 19: 3D Taylor bar impact on a wall. Left: time evolution of the bar length with $\tau_{10}=10^{-7}$ (solid line) and $\tau_{10}=10^{-5}$ (dashed line). Right: analysis of energy conservation in terms of volumetric and elastic energy ( $E_{h}$ and $E_{e}$, respectively), kinetic $\left(E_{k}\right)$ and total $(E)$ energy for $\tau_{10}=10^{-5}$.
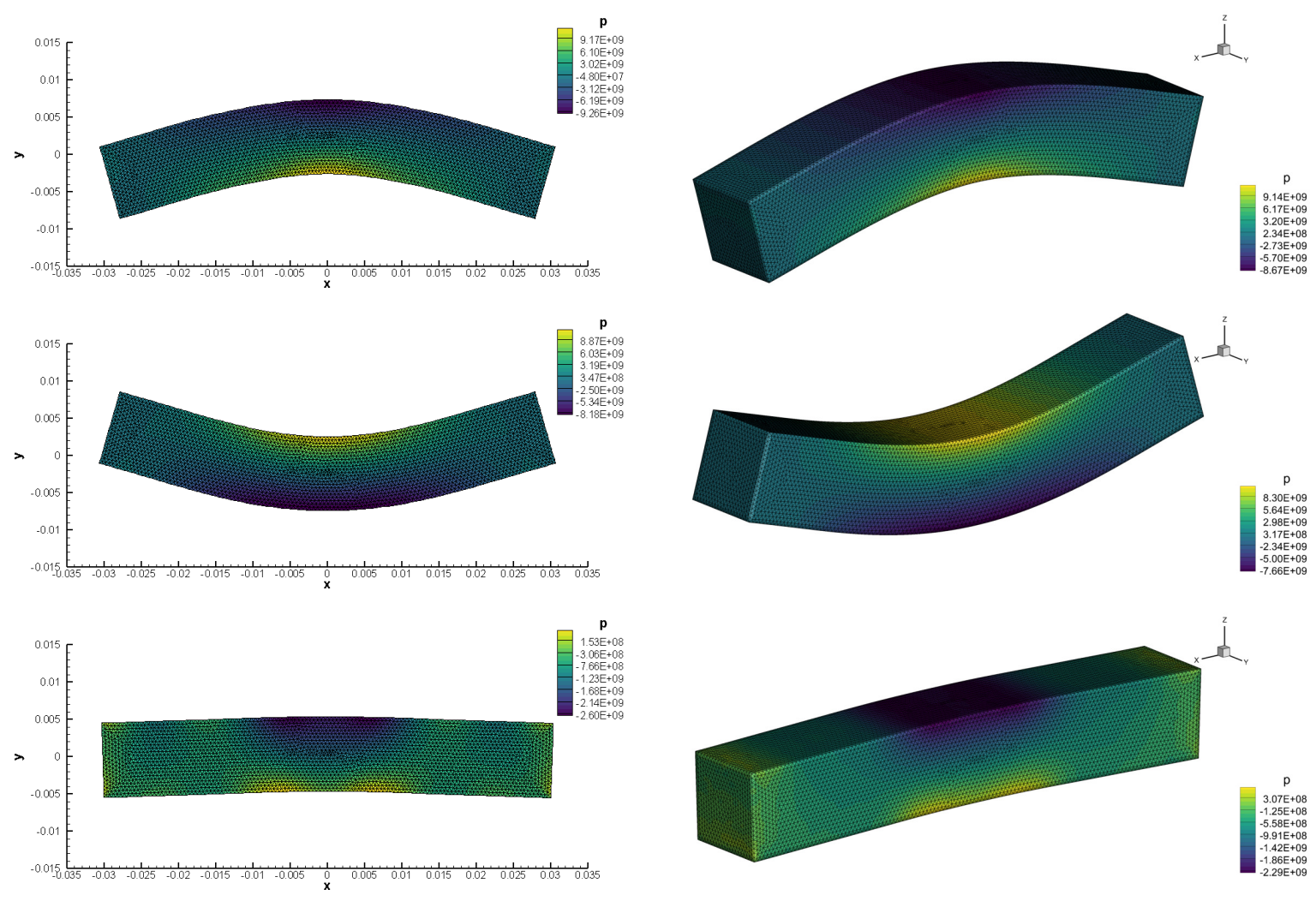

Figure 20: Elastic vibration of a Beryllium plate. Numerical results at output times $t=10^{-5}$ (top), $t=2 \cdot 10^{-5}$ (middle) and $t=3 \cdot 10^{-5}$ (bottom) in $2 \mathrm{D}$ (left) and $3 \mathrm{D}$ (right). 

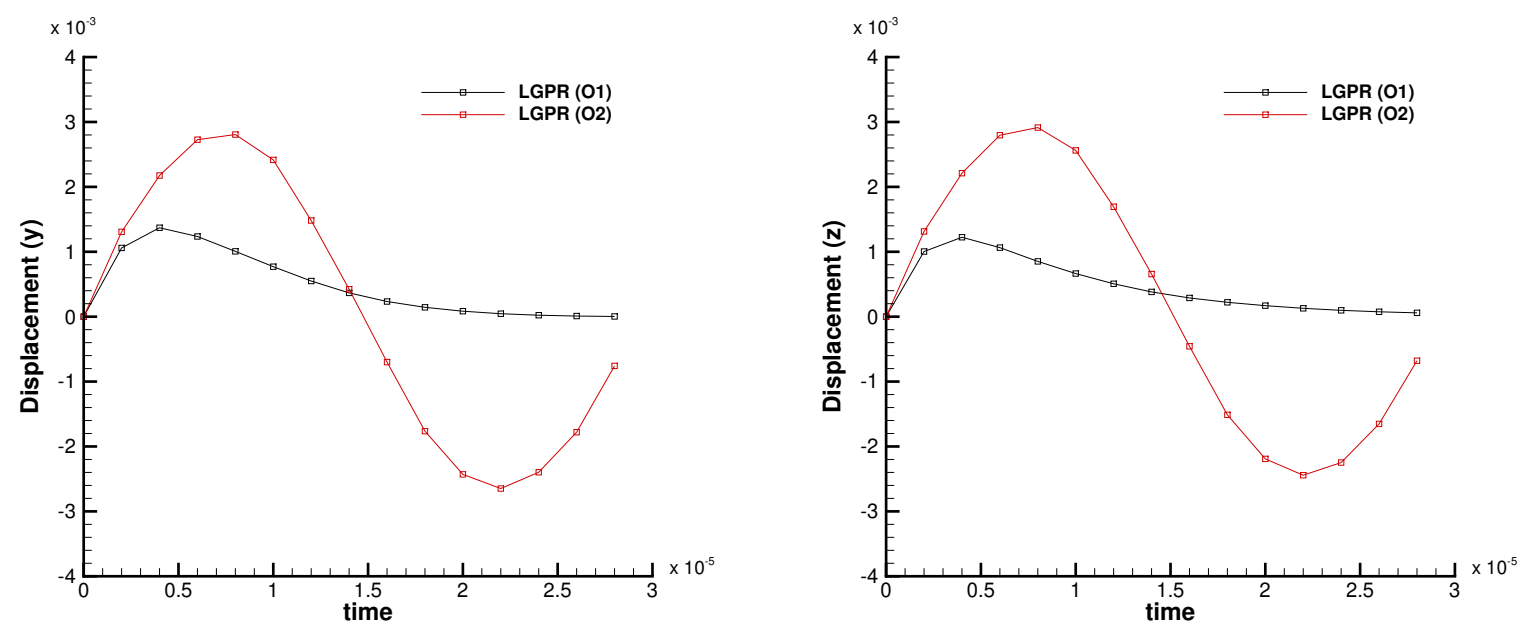

Figure 21: Elastic vibration of a Beryllium plate. Vertical displacement of the point initially located at $\mathbf{x}=(0,0,0)$ with first and second order LGPR scheme.

column is made of $N_{E}=119092$ tetrahedra with characteristic mesh size of $h=1 / 80$. Figure 22 shows the pressure distribution at different output times for the case $\omega_{0}=100$, while Figure 23 collects the results at the same output times for $\omega_{0}=200$. The initial column is represented as a hollow bar for comparison purposes and the expected behaviors are reproduced by the numerical simulation. Notice that there is no spurious oscillations nor nonphysical pressure distribution, thus the results obtained with the LGPR model are in agreement with the literature [23, 73]. The pressure distribution is shown using 21 contour levels in the range $[-5 ; 3] \cdot 10^{6}$.

In Figure 24 we plot the time evolution of the dimensionless height of the column measured at the point initially located at $\mathbf{x}_{T}=(0,0,6)$, showing that the case with $\omega_{0}=200$ exhibits a much stronger distortion and compression of the entire column. Furthermore, the analysis of energy conservation is also reported for both simulations, demonstrating that the total energy is fully conserved by the novel LGPR scheme (35). For comparison purposes, the total energy is normalized to unity for both simulations.

\section{Conclusions}

We have presented a second-order in space and time updated Lagrangian IMEX scheme for the unified firstorder hyperbolic formulation of continuum mechanics, also referred here as the Godunov-Peshkov-Romenski (GPR) model. The novelties of the paper can be summarized as follows. Firstly, for the first time, the GPR model was discretized with an updated Lagrangian scheme. Previously it was treated with either pure Eulerian [47, 20, 28] or total Lagrangian [57] schemes, or even in the Arbitrary-Lagrangian-Eulerian (ALE) framework [90, 21]. Secondly, since the GPR model is equipped with nonlinear relaxation source terms which may become stiff, the Lagrangian scheme is combined with a semi-analytical ad-hoc ODE solver, and second order extension is achieved via IMEX time discretization. This allows us to obtain the numerical solution consistent with the Navier-Stokes-Fourier limit for arbitrarily small relaxation times, thus satisfying the asymptotic preserving property. Moreover, the implicit treatment of the source terms is necessary to achieve better stability properties of the scheme in the stiff cases $\left(\tau_{1} \rightarrow 0\right.$ and $\tau_{2} \rightarrow 0$ ). Thirdly, in this paper, we use a reduced version of the GPR model in which the evolution equation for the distortion field $\boldsymbol{A}_{e}$ is substituted by the evolution equation of its symmetric part only (the metric tensor $\boldsymbol{G}_{e}$ ). This simplification can be used if the rotational degrees of freedom of material particles encoded in the distortion field $\boldsymbol{A}_{e}$ are negligible. The Lagrangian scheme is compatible with the GCL and the study of the asymptotic behavior of the first order fully discrete scheme is derived, demonstrating the asymptotic preserving property exhibited by the new scheme. Furthermore, because the GPR model provides a unified framework for the modeling of various 

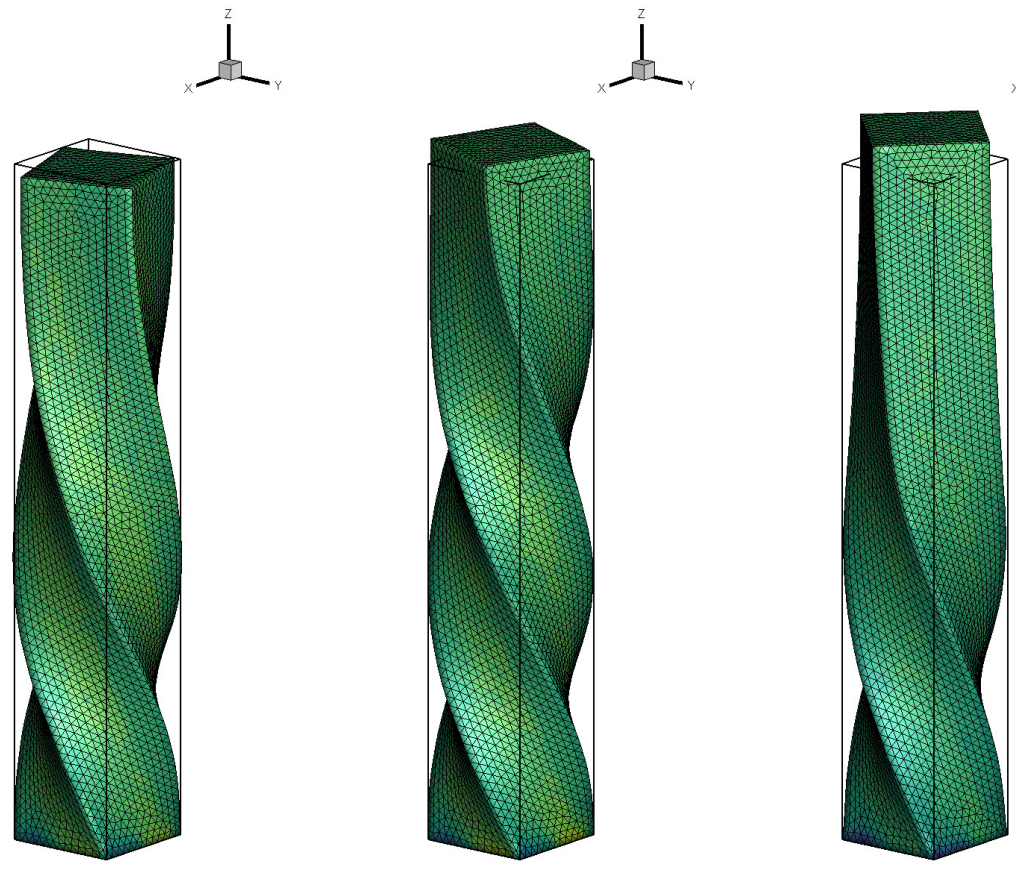

$\underbrace{2}_{1}$

I
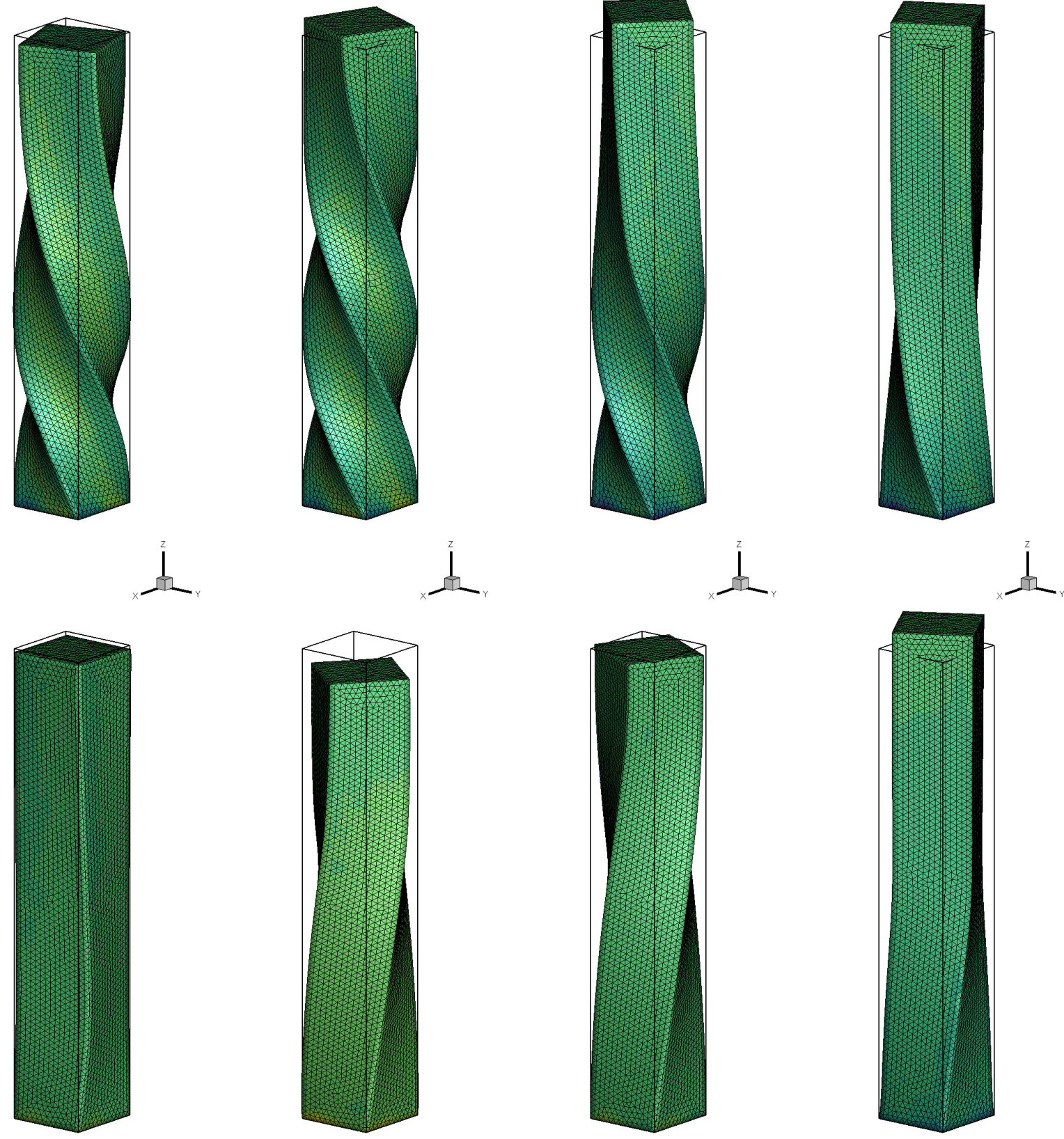

pressure: $\quad-5.00 \mathrm{E}+06 \quad-3.40 \mathrm{E}+06 \quad-1.80 \mathrm{E}+06 \quad-2.00 \mathrm{E}+05 \quad 1.40 \mathrm{E}+06 \quad 3.00 \mathrm{E}+06$

Figure 22: Twisting column with $\omega_{0}=100$. Column shape and pressure distribution at output times $t=0.00375, t=0.075, t=0.1125, t=0.15$, $t=0.1875, t=0.225, t=0.2625$ and $t=0.3$ (from top left to bottom right). The shape is compared with respect to the initial configuration (hollow box). 

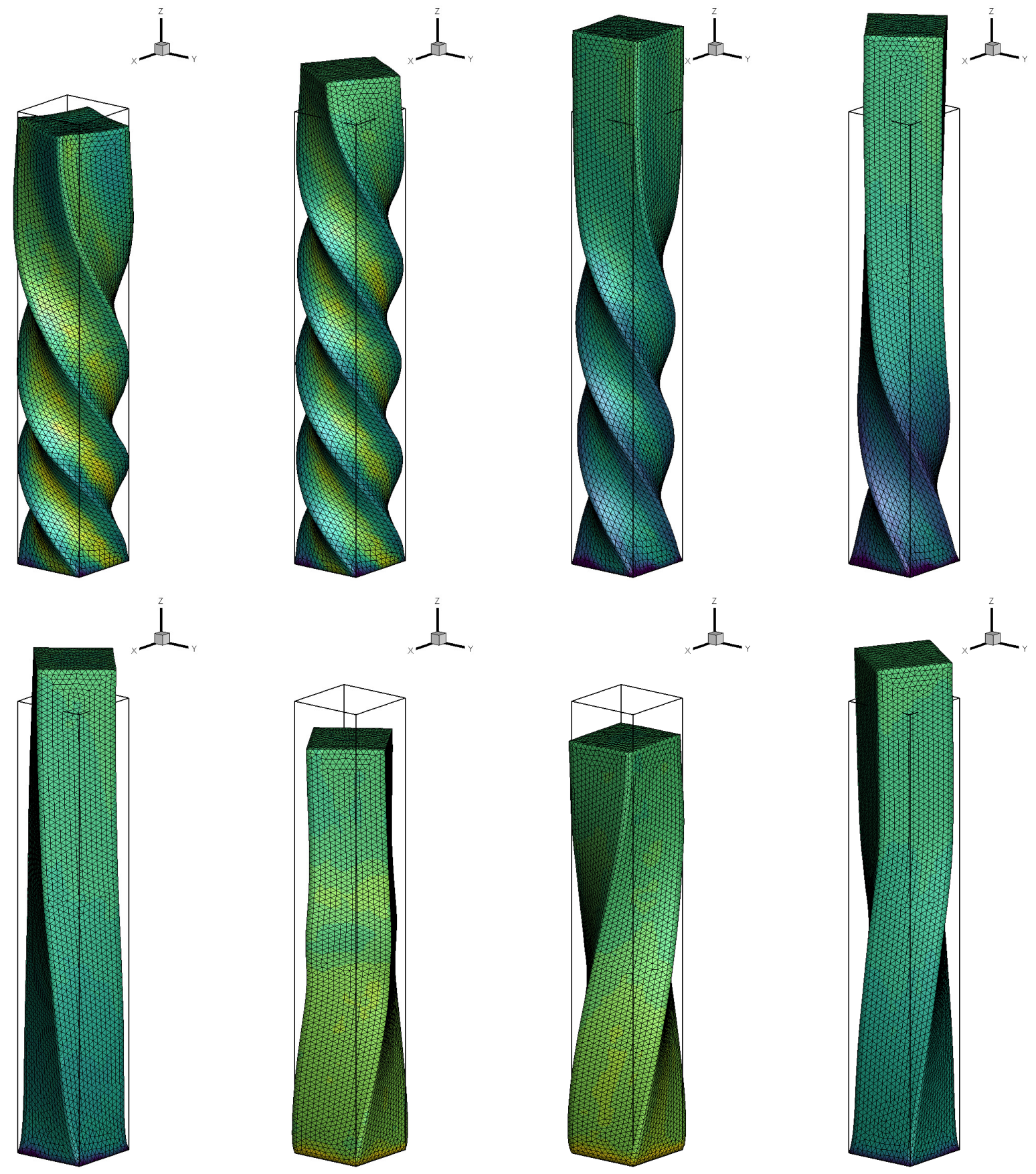

pressure: $\quad-5.00 \mathrm{E}+06 \quad-3.40 \mathrm{E}+06 \quad-1.80 \mathrm{E}+06 \quad-2.00 \mathrm{E}+05 \quad 1.40 \mathrm{E}+06 \quad 3.00 \mathrm{E}+06$

Figure 23: Twisting column with $\omega_{0}=200$. Column shape and pressure distribution at output times $t=0.00375, t=0.075, t=0.1125, t=0.15$, $t=0.1875, t=0.225, t=0.2625$ and $t=0.3$ (from top left to bottom right). The shape is compared with respect to the initial configuration (hollow box). 

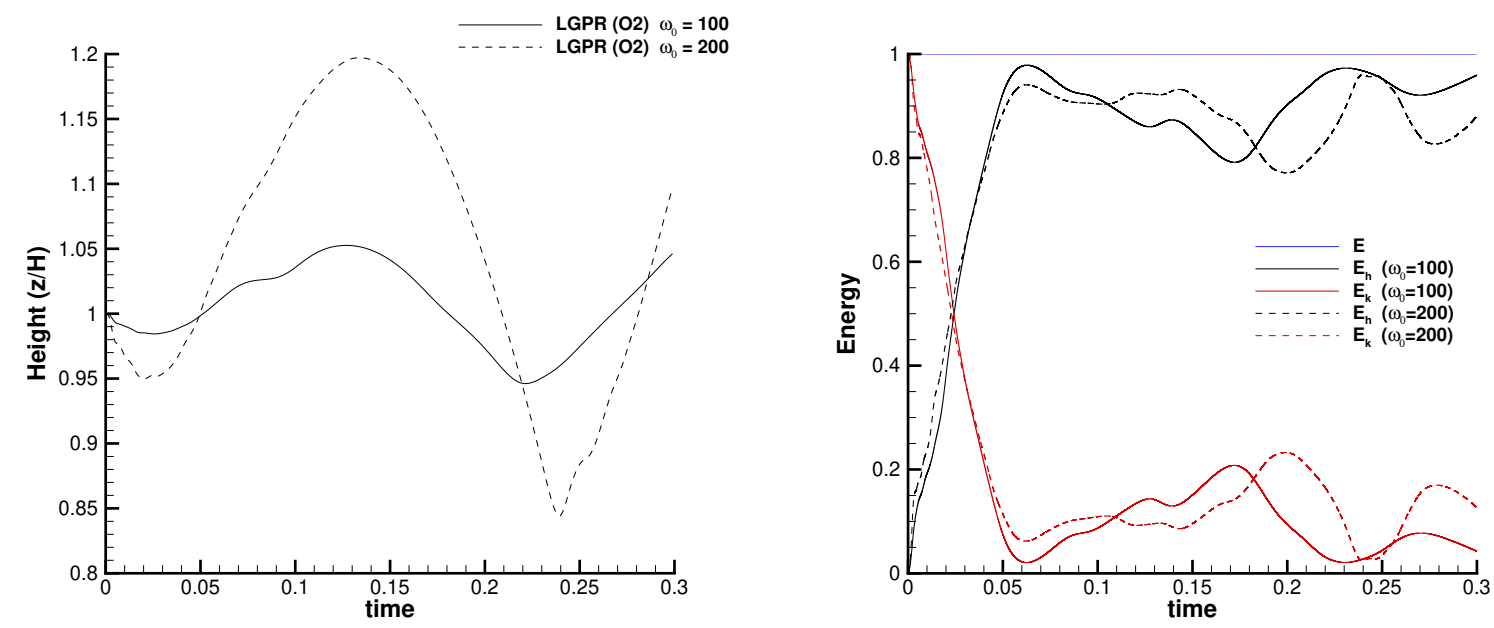

Figure 24: Twisting column. Left: time evolution of non-dimensional height of the column measured at initial point $\mathbf{x}_{T}=(0,0,6)$. Right: analysis of internal $\left(E_{h}\right)$, kinetic $\left(E_{k}\right)$ and total $(E)$ energy conservation normalized to unity for twisting velocity $\omega_{0}=100$ (solid lines) and $\omega_{0}=200$ (dashed lines).

material responses varying from ideal fluids to elastic solids, we have solved an extended series of 2D and 3D test cases covering examples from inviscid hydrodynamics, viscous heat conducting fluids, elastic and elasto-plastic solid mechanics. We emphasize that in all the presented test-cases for fluids and solids the same set of governing equations (2) and the same scheme have been used. Whenever possible, we have compared the numerical solution obtained with the LGPR scheme against analytical solutions of inviscid Euler equations, Navier-Stokes-Fourier equations, and elasto-plasticity theory (e.g. see Section 5.8). A very good agreement between the solutions has been achieved, showing the capability of the scheme of dealing with different material properties. Finally, the new LGPR method collapses to already existing and well-established schemes when approaching the model limits, i.e. the hydrodynamics limit [83] and the ideal hyperelasticity limit [27, 23].

Future work will consist in an extension of the present approach to general unstructured polygonal meshes as well as of generalization towards high-order (higher than 2) schemes which will require accounting for the mesh curvature via evolving the mesh characteristics (e.g. metric tensor) with high-order of accuracy. We also plan to discretize the original GPR model with the evolution equation for the distortion field $\boldsymbol{A}_{e}$ and address the question of constructing structure preserving (e.g. curl-preserving) integration such as discussed in the pure Eulerian [20] and total Lagrangian frameworks [62].

\section{Acknowledgments}

WB and IP would like to thank the Italian Ministry of Instruction, University and Research (MIUR) to support this research with funds coming from PRIN Project 2017 No. 2017KKJP4X entitled "Innovative numerical methods for evolutionary partial differential equations and applications". SC acknowledges the financial support received by the Deutsche Forschungsgemeinschaft (DFG) under the project Droplet Interaction Technologies (DROPIT), grant no. GRK 2160/1.

\section{References}

[1] Miquel Aguirre, Antonio J Gil, Javier Bonet, and Aurelio Arranz. A vertex centred Finite Volume Jameson - Schmidt - Turkel ( JST ) algorithm for a mixed conservation formulation in solid dynamics. Journal of Computational Physics, 259:672-699, 2014.

[2] U. M. Ascher, S. J. Ruuth, and R. J. Spiteri. Implicit-explicit Runge-Kutta methods for time-dependent partial differential equations. Appl. Numer. Math., 25:151-167, 1997. 
[3] T.J. Barth and P.O. Frederickson. Higher order solution of the euler equations on unstructured grids using quadratic reconstruction. 28th Aerospace Sciences Meeting, pages AIAA paper no. 90-0013, January 1990.

[4] T.J. Barth and D.C. Jespersen. The design and application of upwind schemes on unstructured meshes. AIAA Paper 89-0366, pages 1-12, 1989.

[5] P T Barton, D Drikakis, and E I Romenski. An Eulerian finite-volume scheme for large elastoplastic deformations in solids. International Journal for Numerical Methods in Engineering, 81:n/a-n/a, 2009.

[6] Philip T. Barton. An interface-capturing Godunov method for the simulation of compressible solid-fluid problems. Journal of Computational Physics, 390:25-50, aug 2019.

[7] R. Becker. Stosswelle und Detonation. Physik, 8:321, 1923.

[8] M. Ben-Artzi and J. Falcovitz. A second-order godunov-type scheme for compressible fluid dynamics. Journal of Computational Physics, 55:1-32, 1984.

[9] D.J. Benson. Computational methods in lagrangian and eulerian hydrocodes. Computer Methods in Applied Mechanics and Engineering, 99:235-394, 1992.

[10] M. Berndt, J. Breil, S. Galera, M. Kucharik, P.H. Maire, and M. Shashkov. Two-step hybrid conservative remapping for multimaterial arbitrary Lagrangian-Eulerian methods. Journal of Computational Physics, 230:6664-6687, 2011.

[11] Barry Bernstein. Hypo-elasticity and elasticity. Arch. Rational Mech. Anal., 6:89-104, 1960. https://doi.org/10.1007/BF00276156.

[12] J. Bonet, A. J. Gil, C. Hean Lee, M. Aguirre, and R. Ortigosa. A first order hyperbolic framework for large strain computational solid dynamics. part I: Total Lagrangian isothermal elasticity. Computer Methods in Applied Mechanics and Engineering, 283:689-732, January 2015 .

[13] J. Bonet, C. Hean Lee, A. J. Gil, and A. Ghavamian. A first order hyperbolic framework for large strain computational solid dynamics. part III: Thermo-elasticity. Computer Methods in Applied Mechanics and Engineering, 373:113505, January 2021.

[14] S. Boscarino and G. Russo. On a class of uniformly accurate IMEX Runge-Kutta schemes and applications to hyperbolic systems with relaxation. SIAM J. Sci. Comput., 31:1926-1945, 2009.

[15] W. Boscheri. High order direct arbitrary-lagrangian-eulerian (ale) finite volume schemes for hyperbolic systems on unstructured meshes. Archives of Computational Methods in Engineering, 24:751-801, 2017.

[16] W. Boscheri, G. Dimarco, R. Loubère, M. Tavelli, and M.H. Vignal. A second order all Mach number IMEX finite volume solver for the three dimensional Euler equations. J. Comp. Phys., 415:109486, 2020.

[17] W. Boscheri, G. Dimarco, and M. Tavelli. An efficient second order all Mach finite volume solver for the compressible Navier-Stokes equations. Computer Methods in Applied Mechanics and Engineering, 374:113602, 2021.

[18] W. Boscheri and M. Dumbser. Arbitrary-Lagrangian-Eulerian One-Step WENO Finite Volume Schemes on Unstructured Triangular Meshes. Communications in Computational Physics, 14:1174-1206, 2013.

[19] W. Boscheri and M. Dumbser. A direct Arbitrary-Lagrangian-Eulerian ADER-WENO finite volume scheme on unstructured tetrahedral meshes for conservative and non-conservative hyperbolic systems in 3D. J. Comput. Phys., 275:484-523, 2014.

[20] W. Boscheri, M. Dumbser, M. Ioriatti, I. Peshkov, and E. Romenski. A structure-preserving staggered semi-implicit finite volume scheme for continuum mechanics. Journal of Computational Physics, 424:109866, jan 2021.

[21] W. Boscheri, M. Dumbser, and R. Loubère. Cell centered direct Arbitrary-Lagrangian-Eulerian ADER-WENO finite volume schemes for nonlinear hyperelasticity. Computers and Fluids, 134-135:111-129, 2016.

[22] W. Boscheri, M. Dumbser, R. Loubère, and P.-H. Maire. A second-order cell-centered Lagrangian ADER-MOOD finite volume scheme on multidimensional unstructured meshes for hydrodynamics. J. Comput. Phys., 358:103 - 129, 2018.

[23] Walter Boscheri, Raphaël Loubère, and Pierre-Henri Maire. A cell-centered Lagrangian ADER-MOOD finite volume scheme on unstructured meshes for a class of hyper-elasticity models. apr 2021.

[24] Walter Boscheri and Lorenzo Pareschi. High order pressure-based semi-implicit imex schemes for the $3 \mathrm{~d}$ navier-stokes equations at all mach numbers. Journal of Computational Physics, 434:110206, 2021.

[25] A. Bourgeade, P. LeFloch, and P.A. Raviart. An asymptotic expansion for the solution of the generalized riemann problem. Part II: application to the gas dynamics equations. Annales de l'institut Henri Poincaré (C) Analyse non linéaire, 6:437-480, 1989.

[26] J. Breil, S. Galera, and P.H. Maire. Multi-material ALE computation in inertial confinement fusion code CHIC. Computers and Fluids, 46:161-167, 2011.

[27] J. Breil, G. Georges, and P.-H. Maire. 3D cell-centered Lagrangian second order scheme for the numerical modeling of hyperelasticity system. Computer and Fluids, 207:104523, 2020.

[28] Saray Busto, Simone Chiocchetti, Michael Dumbser, Elena Gaburro, and Ilya Peshkov. High Order ADER Schemes for Continuum Mechanics. Frontiers in Physics, 8(32), mar 2020.

[29] E.J. Caramana, D.E. Burton, M.J. Shashkov, and P.P. Whalen. The construction of compatible hydrodynamics algorithms utilizing conservation of total energy. Journal of Computational Physics, 146:227-262, 1998.

[30] E.J. Caramana, C.L. Rousculp, and D.E. Burton. A compatible, energy and symmetry preserving Lagrangian hydrodynamics algorithm in three-dimensional Cartesian geometry. Journal of Computational Physics, 157:89 - 119, 2000.

[31] G. Carré, S. Del Pino, B. Després, and E. Labourasse. A cell-centered Lagrangian hydrodynamics scheme on general unstructured meshes in arbitrary dimension. Journal of Computational Physics, 228:5160-5183, 2009.

[32] J. Cheng and C.W. Shu. A high order ENO conservative Lagrangian type scheme for the compressible Euler equations. Journal of Computational Physics, 227:1567-1596, 2007.

[33] J. Cheng and C.W. Shu. A cell-centered Lagrangian scheme with the preservation of symmetry and conservation properties for compressible fluid flows in two-dimensional cylindrical geometry. Journal of Computational Physics, 229:7191-7206, 2010.

[34] J. Cheng and C.W. Shu. Improvement on spherical symmetry in two-dimensional cylindrical coordinates for a class of control volume Lagrangian schemes. Communications in Computational Physics, 11:1144-1168, 2012.

[35] Jun-Bo Cheng, Yueling Jia, Song Jiang, Eleuterio F. Toro, and Ming Yu. A second-order cell-centered Lagrangian method for twodimensional elastic-plastic flows. Communications in Computational Physics, 22(5):1224-1257, 2017. 
[36] S. Chiocchetti and C. Müller. A Solver for Stiff Finite-Rate Relaxation in Baer-Nunziato Two-Phase Flow Models. Fluid Mechanics and its Applications, 121:31-44, 2020.

[37] A. Claisse, B. Després, E.Labourasse, and F. Ledoux. A new exceptional points method with application to cell-centered Lagrangian schemes and curved meshes. Journal of Computational Physics, 231:4324-4354, 2012.

[38] B. Després. Numerical Methods for Eulerian and Lagrangian Conservation Laws. Frontiers in Mathematics. Springer International Publishing, 2017.

[39] B. Després and C. Mazeran. Symmetrization of Lagrangian gas dynamic in dimension two and multimdimensional solvers. C.R. Mecanique, 331:475-480, 2003.

[40] B. Després and C. Mazeran. Lagrangian gas dynamics in two dimensions and Lagrangian systems. Arch. Rational Mech. Anal., 178:327$372,2005$.

[41] V.A. Dobrev, T.E. Ellis, Tz.V. Kolev, and R.N. Rieben. Curvilinear Finite elements for Lagrangian hydrodynamics. International Journal for Numerical Methods in Fluids, 65:1295-1310, 2011.

[42] V.A. Dobrev, T.E. Ellis, Tz.V. Kolev, and R.N. Rieben. High Order Curvilinear Finite Elements for Lagrangian Hydrodynamics. SIAM Journal on Scientific Computing, 34:606-641, 2012.

[43] V.A. Dobrev, T.E. Ellis, Tz.V. Kolev, and R.N. Rieben. High Order Curvilinear Finite Elements for axisymmetric Lagrangian Hydrodynamics. Computers and Fluids, 83:58-69, 2013.

[44] J.K. Dukovicz and B. Meltz. Vorticity errors in multidimensional Lagrangian codes. Journal of Computational Physics, $99: 115$ - 134 , 1992.

[45] M. Dumbser and M. Kaeser. Arbitrary high order non-oscillatory finite volume schemes on unstructured meshes for linear hyperbolic systems. Journal of Computational Physics, 221:693 - 723, 2007.

[46] Michael Dumbser, Ilya Peshkov, and Evgeniy Romenski. A unified hyperbolic formulation for viscous fluids and elastoplastic solids. In Christian Klingenberg and Michael Westdickenberg, editors, Springer Proceedings in Mathematics and Statistics, volume 237 of Springer Proceedings in Mathematics and Statistics, pages 451-463. Springer International Publishing, 2018.

[47] Michael Dumbser, Ilya Peshkov, Evgeniy Romenski, and Olindo Zanotti. High order ADER schemes for a unified first order hyperbolic formulation of continuum mechanics: Viscous heat-conducting fluids and elastic solids. Journal of Computational Physics, 314:824-862, jun 2016.

[48] Michael Dumbser, Ilya Peshkov, Evgeniy Romenski, and Olindo Zanotti. High order ADER schemes for a unified first order hyperbolic formulation of Newtonian continuum mechanics coupled with electro-dynamics. Journal of Computational Physics, 348:298-342, nov 2017.

[49] D. P. Flanagan and T. Belytschko. A uniform strain hexahedron and quadrilateral with orthogonal hourglass control. International Journal for Numerical Methods in Engineering, 17(5):679-706, 1981.

[50] P. Le Floch and P.A. Raviart. An asymptotic expansion for the solution of the generalized riemann problem. Part I: General theory. Annales de l'institut Henri Poincaré (C) Analyse non linéaire, 5:179-207, 1988.

[51] F.Vilar. Cell-centered discontinuous Galerkin discretization for two-dimensional Lagrangian hydrodynamics. Computers and Fluids, 64:6473, 2012.

[52] F.Vilar, P.H. Maire, and R. Abgrall. Cell-centered discontinuous Galerkin discretizations for two-dimensional scalar conservation laws on unstructured grids and for one-dimensional Lagrangian hydrodynamics. Computers and Fluids, 46(1):498-604, 2010.

[53] S.L. Gavrilyuk, N. Favrie, and R. Saurel. Modelling wave dynamics of compressible elastic materials. Journal of Computational Physics, 227:2941-2969, 2008.

[54] G. Georges, J. Breil, and P.-H. Maire. A 3D GCL compatible cell-centered Lagrangian scheme for solving gas dynamics equations. J. Comput. Phys., 305:921-941, 2016.

[55] A. J. Gil, C. Hean Lee, J. Bonet, and R. Ortigosa. A first order hyperbolic framework for large strain computational solid dynamics. part II: Total Lagrangian compressible, nearly incompressible and truly incompressible elasticity. Computer Methods in Applied Mechanics and Engineering, 300:146-181, March 2016.

[56] S K Godunov. Elements of mechanics of continuous media. Nauka, 1st russian edition, 1978.

[57] S. K. Godunov and I. M. Peshkov. Thermodynamically consistent nonlinear model of elastoplastic Maxwell medium. Computational Mathematics and Mathematical Physics, 50(8):1409-1426, aug 2010.

[58] S. K. Godunov and E. I. Romenskii. Nonstationary equations of nonlinear elasticity theory in eulerian coordinates. Journal of Applied Mechanics and Technical Physics, 13(6):868-884, nov 1972.

[59] S K Godunov and E I Romenskii. Elements of continuum mechanics and conservation laws. Kluwer Academic/Plenum Publishers, 2003.

[60] G.L. Goudreau and J.O. Hallquist. Recent developments in large-scale finite element Lagrangian hydrocode technology. Computer Methods in Applied Mechanics and Engineering, 33(1):725-757, 1982.

[61] J. Haider, C. Hean Lee, A. J. Gil, A. Huerta, and J. Bonet. An upwind cell centred total Lagrangian finite volume algorithm for nearly incompressible explicit fast solid dynamic applications. Computer Methods in Applied Mechanics and Engineering, 340:684 - 727, 2018.

[62] Osama I. Hassan, Ataollah Ghavamian, Chun Hean Lee, Antonio J. Gil, Javier Bonet, and Ferdinando Auricchio. An upwind vertex centred finite volume algorithm for nearly and truly incompressible explicit fast solid dynamic applications: Total and Updated Lagrangian formulations. Journal of Computational Physics: X, 3:100025, jun 2019.

[63] B.P. Howell and G.J. Ball. A Free-Lagrange Augmented Godunov Method for the Simulation of Elastic-Plastic Solids. Journal of Computational Physics, 175(1):128-167, jan 2002.

[64] C. Hu and C.W. Shu. Weighted essentially non-oscillatory schemes on triangular meshes. J. Comp. Phys., 150:97-127, 1999.

[65] Haran Jackson and Nikos Nikiforakis. A numerical scheme for non-Newtonian fluids and plastic solids under the GPR model. Journal of Computational Physics, 387:410-429, jun 2019.

[66] Haran Jackson and Nikos Nikiforakis. A unified Eulerian framework for multimaterial continuum mechanics. Journal of Computational Physics, 401:109022, jan 2020.

[67] J. Kamm, J. Brock, S. Brandon, D.L. Cotrell, B.M. Johnson, P. Knupp, T.G. Trucano, W.J. Rider, and V.G. Weirs. Enhanced verification test suite for physics simulations codes. Technical Report LA-14379, 2008. 
[68] J.R. Kamm and F.X. Timmes. On efficient generation of numerically robust sedov solutions. Technical Report LA-UR-07-2849, Los Alamos National Laboratory, 2007.

[69] M. Käser and A. Iske. ADER schemes on adaptive triangular meshes for scalar conservation laws. Journal of Computational Physics, 205:486-508, 2005.

[70] Friedemann Kemm, Elena Gaburro, Ferdinand Thein, and Michael Dumbser. A simple diffuse interface approach for compressible flows around moving solids of arbitrary shape based on a reduced Baer-Nunziato model. Computers $\mathcal{E}$ Fluids, 204:104536, may 2020.

[71] R.E. Kidder. Laser-driven compression of hollow shells: power requirements and stability limitations. Nucl. Fus., 1:3 - 14, 1976.

[72] G. Kluth and B. Després. Discretization of hyperelasticity on unstructured mesh with a cell-centered Lagrangian scheme. Journal of Computational Physics, 229(24):9092 - 9118, 2010.

[73] C.H. Lee, A.J. Gil, and J. Bonet. Development of a stabilised Petrov-Galerkin formulation for conservation laws in Lagrangian fast solid dynamics. Comput. Methods Appl. Mech. Engrg., 268:40-64, 2014.

[74] E.J. Lieberman, X. Liu, N.R. Morgan, D.J. Luscher, and D.E. Burton. A higher-order Lagrangian discontinuous Galerkin hydrodynamic method for solid dynamics. Comp. Meth. Appl. Mech. Engrg., 353:467-490, 2019.

[75] E.J. Lieberman, N.R. Morgan, D.J. Luscher, and D.E. Burton. A higher-order Lagrangian discontinuous Galerkin hydrodynamic method for elastic-plastic flows. Comput. Math. Appl., 353:318-334, 2019.

[76] W. Liu, J. Cheng, and C.W. Shu. High order conservative Lagrangian schemes with Lax-Wendroff type time discretization for the compressible Euler equations. Journal of Computational Physics, 228:8872-8891, 2009.

[77] R. Loubère, P.H. Maire, and P. Váchal. A second-order compatible staggered Lagrangian hydrodynamics scheme using a cell-centered multidimensional approximate Riemann solver. Procedia Computer Science, 1:1931-1939, 2010.

[78] R. Loubère, P.H. Maire, and P. Váchal. 3D staggered Lagrangian hydrodynamics scheme with cell-centered Riemann solver-based artificial viscosity. International Journal for Numerical Methods in Fluids, 72:22 - 42, 2013.

[79] P.-H. Maire. A high-order cell-centered Lagrangian scheme for compressible fluid flows in two-dimensional cylindrical geometry. $J$. Comput. Phys., 228(18):6882-6915, 2009.

[80] P.-H. Maire. A unified sub-cell force-based discretization for cell-centered Lagrangian hydrodynamics on polygonal grids. Int. J. Numer. Meth. Fluid, 65:1281-1294, 2011.

[81] P.-H. Maire. A high-order one-step sub-cell force-based discretization for cell-centered Lagrangian hydrodynamics on polygonal grids. Computer and Fluids, 46(1):341-347, 2011.

[82] P.-H. Maire, R. Abgrall, J. Breil, R. Loubère, and B. Rebourcet. A nominally second-order cell-centered Lagrangian scheme for simulating elastic-plastic flows on two-dimensional unstructured grids. J. Comput. Phys., 235:626-665, 2013.

[83] P.-H. Maire, R. Abgrall, J. Breil, and J. Ovadia. A cell-centered Lagrangian scheme for two-dimensional compressible flow problems. SIAM Journal on Scientific Computing, 29:1781-1824, 2007.

[84] P.-H. Maire and B. Nkonga. Multi-scale Godunov-type method for cell-centered discrete Lagrangian hydrodynamics. J. Comput. Phys., 228(3):799-821, 2009.

[85] A N Malyshev and E I Romenskii. Hyperbolic equations for heat transfer. Global solvability of the Cauchy problem. Siberian Mathematical Journal, 27(5):734-740, 1987.

[86] L. Anand M.E. Gurtin, E. Fried. The mechanics and thermodynamics of continua. Cambridge University Press, 2009.

[87] C.D. Munz. On Godunov-type schemes for Lagrangian gas dynamics. SIAM Journal on Numerical Analysis, 31:17-42, 1994.

[88] A. López Ortega and G. Scovazzi. A geometrically-conservative, synchronized, flux-corrected remap for arbitrary Lagrangian-Eulerian computations with nodal finite elements. Journal of Computational Physics, 230:6709-6741, 2011.

[89] L. Pareschi and G. Russo. Implicit-explicit runge-kutta schemes and applications to hyperbolic systems with relaxation. J. Sci. Comput., 25:129-155, 2005

[90] Ilya Peshkov, Walter Boscheri, Raphaël Loubère, Evgeniy Romenski, and Michael Dumbser. Theoretical and numerical comparison of hyperelastic and hypoelastic formulations for Eulerian non-linear elastoplasticity. Journal of Computational Physics, 387:481-521, 2019.

[91] Ilya Peshkov, Michael Dumbser, Walter Boscheri, Evgeniy Romenski, Simone Chiocchetti, and Matteo Ioriatti. Simulation of nonNewtonian viscoplastic flows with a unified first order hyperbolic model and a structure-preserving semi-implicit scheme. Computers $\mathcal{E}$ Fluids, 224:104963, jun 2021.

[92] Ilya Peshkov, Michal Pavelka, Evgeniy Romenski, and Miroslav Grmela. Continuum mechanics and thermodynamics in the Hamilton and the Godunov-type formulations. Continuum Mechanics and Thermodynamics, 30(6):1343-1378, nov 2018.

[93] Ilya Peshkov and Evgeniy Romenski. A hyperbolic model for viscous Newtonian flows. Continuum Mechanics and Thermodynamics, 28(1-2):85-104, mar 2016

[94] Ilya Peshkov, Evgeniy Romenski, and Michael Dumbser. Continuum mechanics with torsion. Continuum Mechanics and Thermodynamics, 31(5):1517-1541, 2019.

[95] Evgeniy Romenski, Ilya Peshkov, Michael Dumbser, Francesco Fambri, Fambri, and Francesco. A new continuum model for general relativistic viscous heat-conducting media. Philosophical Transactions of the Royal Society A: Mathematical, Physical and Engineering Sciences, 378(2170):20190175, may 2020.

[96] E. I. Romenskii. Dynamic three-dimensional equations of the Rakhmatulin elastic-plastic model. Journal of Applied Mechanics and Technical Physics, 20(2):229-244, 1979.

[97] E I Romenskii. Hyperbolic equations of Maxwell's nonlinear model of elastoplastic heat-conducting media. Siberian Mathematical Journal, 30(4):606-625, 1990.

[98] M B Rubin. An Eulerian formulation of inelasticity: from metal plasticity to growth of biological tissues. Philosophical Transactions of the Royal Society A: Mathematical, Physical and Engineering Sciences, 377(2144):20180071, may 2019.

[99] V. V. Rusanov. Calculation of Interaction of Non-Steady Shock Waves with Obstacles. J. Comput. Math. Phys. USSR, 1:267-279, 1961

[100] J. Massoni S. Hank, N. Favrie. Modeling hyperelasticity in non-equilibrium multiphase flows. Journal of Computational Physics, 330:6591, 2017.

[101] S.K. Sambasivan, M.J. Shashkov, and D.E. Burton. A finite volume cell-centered Lagrangian hydrodynamics approach for solids in general 
unstructured grids. Int. J. Numer. Meth. Fluid, 72:770-810, 2013.

[102] G. Scovazzi. Lagrangian shock hydrodynamics on tetrahedral meshes: A stable and accurate variational multiscale approach. Journal of Computational Physics, 231:8029-8069, 2012.

[103] G. Scovazzi, B. Carnes, X. Zeng, and S. Rossi. A simple, stable, and accurate linear tetrahedral finite element for transient, nearly, and fully incompressible solid dynamics: a dynamic variational multiscale approach. International Journal for Numerical Methods in Engineering, 106:799-839, 2016.

[104] C.-W. Shu and J. Cheng. A Third Order Conservative Lagrangian Type Scheme on Curvilinear Meshes for the Compressible Euler Equations. Commun. Comput. Phys., 4:1008-1024, 2008.

[105] S.K.Godunov. A finite difference method for the computation of discontinuous solutions of the equations of fluid dynamics. Mat. Sbornik, 47:357-393, 1959.

[106] R.W. Smith. AUSM(ALE): a geometrically conservative arbitrary lagrangian-eulerian flux splitting scheme. Journal of Computational Physics, 150:268-286, 1999.

[107] M. Tavelli, E. Romenski, S. Chiocchetti, A.-A. Gabriel, and M. Dumbser. Space-time adaptive ADER discontinuous Galerkin schemes for nonlinear hyperelasticity with material failure. Journal of Computational Physics, page 109758, 2020.

[108] Maurizio Tavelli, Michael Dumbser, Dominic Etienne Charrier, Leonhard Rannabauer, Tobias Weinzierl, and Michael Bader. A simple diffuse interface approach on adaptive cartesian grids for the linear elastic wave equations with complex topography. Journal of Computational Physics, 386:158-189, 2019.

[109] G.I. Taylor. The use of flat-ended projectiles for determining dynamic yield stress i. theoretical considerations. Proceedings of the Royal Society of London A: Mathematical, Physical and Engineering Sciences, 194(1038):289-299, 1948.

[110] V.A. Titarev and E.F. Toro. ADER: Arbitrary high order Godunov approach. Journal of Scientific Computing, 17(1-4):609-618, December 2002.

[111] V.A. Titarev and E.F. Toro. ADER schemes for three-dimensional nonlinear hyperbolic systems. Journal of Computational Physics, 204:715-736, 2005

[112] E. F. Toro and V. A. Titarev. Derivative Riemann solvers for systems of conservation laws and ADER methods. Journal of Computational Physics, 212(1):150-165, 2006.

[113] E.F. Toro. Anomalies of conservative methods: analysis, numerical evidence and possible cures. International Journal of Computational Fluid Dynamics, 11:128-143, 2002.

[114] E.F. Toro. Riemann Solvers and Numerical Methods for Fluid Dynamics: a Practical Introduction. Springer, 2009.

[115] C. Truesdell. Hypo-elasticity. Journal of Rational Mechanics and Analysis, 4:83-1020, 1955.

[116] J. von Neumann and R.D. Richtmyer. A method for the numerical calculations of hydrodynamical shocks. 21:232-238, 1950

[117] Tim Wallis, Philip T. Barton, and Nikolaos Nikiforakis. A flux-enriched Godunov method for multi-material problems with interface slide and void opening. Journal of Computational Physics, 442:110499, oct 2021

[118] M.L. Wilkins. Calculation of elastic plastic flow. In B. Alder, S. Fernbach, and M. Rotenberg, editors, Methods in Computational Physics, volume 3, pages 211-263. Academic Press, New York, 1964.

[119] T. Wu, M. Shashkov, N.R. Morgan, D. Kuzmin, and H. Luo. An updated Lagrangian discontinuous Galerkin hydrodynamic method for gas dynamics. Comput. Math. Appl., 78:258-273, 2019. 